\title{
Validation of M-C/H-C Bond Enthalpy Relationship through Application of Density Functional Theory
}

\author{
Eric Clot, Claire Mégret, Odile Eisenstein, Robin N. Perutz.
}

\section{Supporting Information}

Complete list of authors for reference 18:

Gaussian 98, Revision A.11, M. J. Frisch, G. W. Trucks, H. B. Schlegel, G. E. Scuseria, M. A. Robb, J. R. Cheeseman, V. G. Zakrzewski, J. A. Montgomery, Jr., R. E. Stratmann, J. C. Burant, S. Dapprich, J. M. Millam, A. D. Daniels, K. N. Kudin, M. C. Strain, O. Farkas, J. Tomasi, V. Barone, M. Cossi, R. Cammi, B. Mennucci, C. Pomelli, C. Adamo, S. Clifford, J. Ochterski, G. A. Petersson, P. Y. Ayala, Q. Cui, K. Morokuma, P. Salvador, J. J. Dannenberg, D. K. Malick, A. D. Rabuck, K. Raghavachari, J. B. Foresman, J. Cioslowski, J. V. Ortiz, A. G. Baboul, B. B. Stefanov, G. Liu, A. Liashenko, P. Piskorz, I. Komaromi, R. Gomperts, R. L. Martin, D. J. Fox, T. Keith, M. A. Al-Laham, C. Y. Peng, A. Nanayakkara, M. Challacombe, P. M. W. Gill, B. Johnson, W. Chen, M. W. Wong, J. L. Andres, C. Gonzalez, M. Head-Gordon, E. S. Replogle, and J. A. Pople, Gaussian, Inc., Pittsburgh PA, 2001.

Figure S1. Correlation between calculated and experimental C-H bond enthalpies using BP86 functional and CRC Handbook $84^{\text {th }}$ edition, respectively.

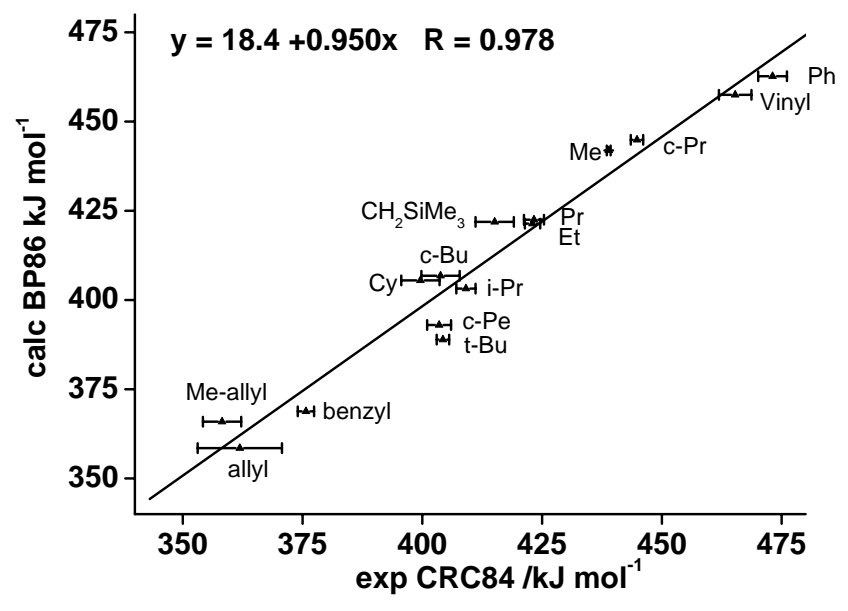


Figure S2. Correlations for the Wick and Jones series with linear (black) and quadratic (blue) fits. (a) Experimental $\Delta H(R h-$ C) (relative to phenyl) $v s$ experimental $\Delta \mathrm{H}(\mathrm{H}-\mathrm{C})$. (b) BP86 calculated $\Delta \mathrm{H}(\mathrm{Rh}-\mathrm{C}) v s$ calculated $\Delta \mathrm{H}(\mathrm{H}-\mathrm{C})$. (c) Calculated (BP86) $\Delta \mathrm{H}(\mathrm{Rh}-\mathrm{C})$ vs experimental $\Delta \mathrm{H}(\mathrm{Rh}-\mathrm{C})$.

Wick and Jones Series BP86
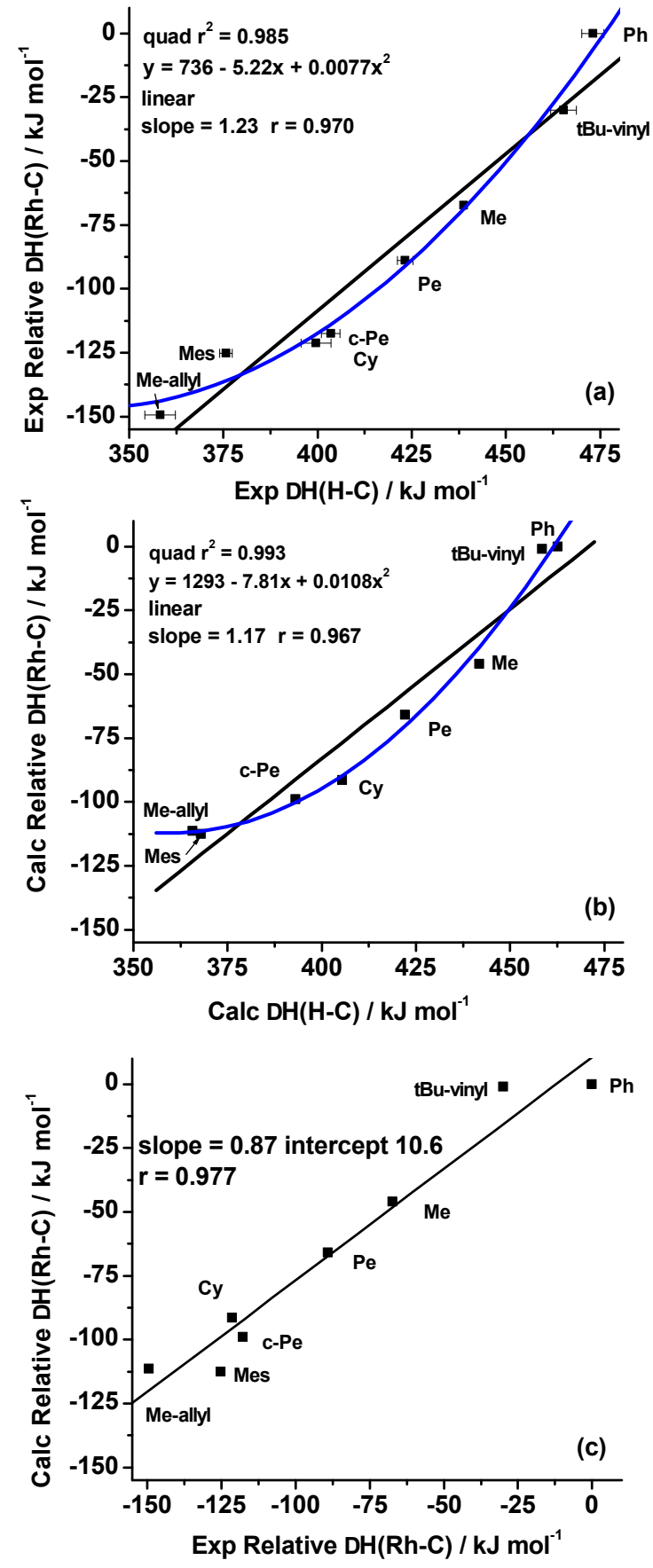
Figure S3. Correlations for the Bennett and Wolczanski system with benzyl and phenyl excluded from correlation lines. (a) Experimental $\Delta \mathrm{H}(\mathrm{Ti}-\mathrm{C})$ (relative to phenyl) $v s$ experimental $\Delta \mathrm{H}(\mathrm{H}-\mathrm{C})$. (b) B3PW91 calculated $\Delta \mathrm{H}(\mathrm{Ti}-\mathrm{C}) v s$ calculated $\Delta \mathrm{H}(\mathrm{H}-$ C). The added points shown in red are not included in the correlation. (c) Calculated $\Delta \mathrm{H}(\mathrm{Ti}-\mathrm{C}) v s$ experimental $\Delta \mathrm{H}(\mathrm{Rh}-\mathrm{C})$.
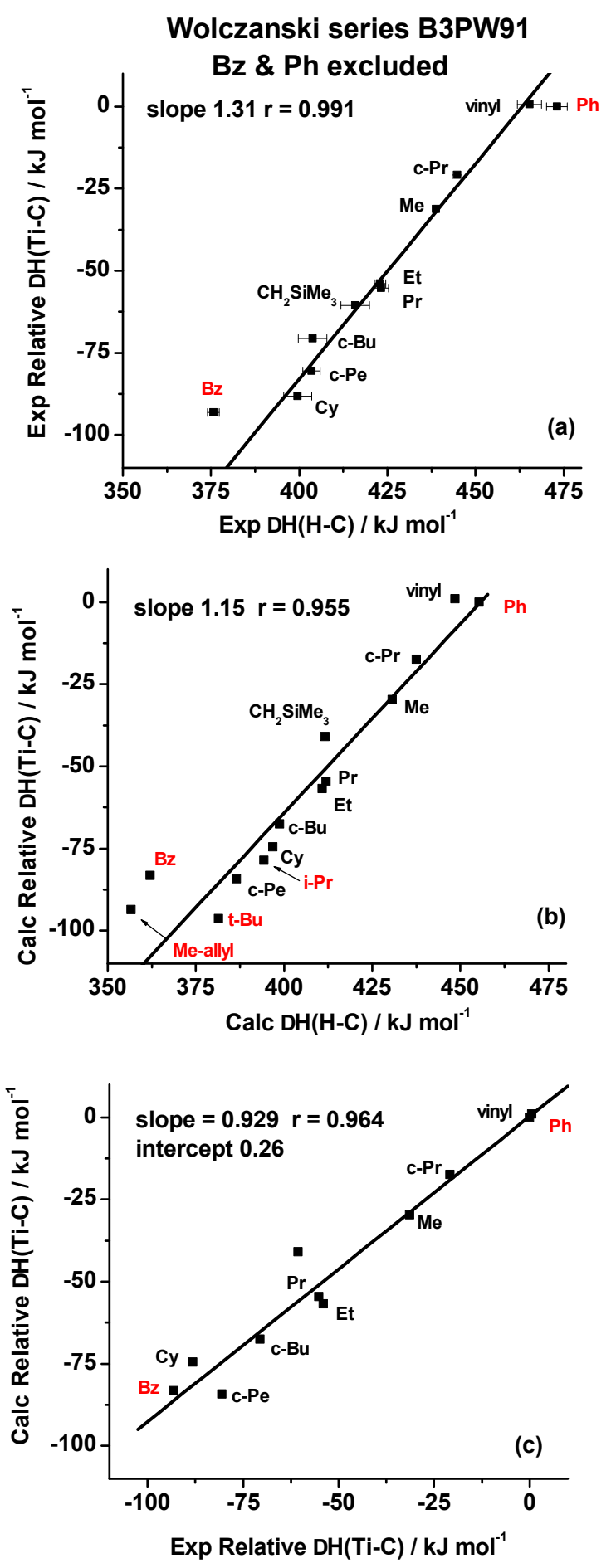
Figure S4. Correlations for the Bennett and Wolczanski system. (a) Experimental $\Delta \mathrm{H}(\mathrm{Ti}-\mathrm{C})$ (relative to phenyl) $v s$ experimental $\Delta \mathrm{H}(\mathrm{H}-\mathrm{C})$. (b) BP86 calculated $\Delta \mathrm{H}(\mathrm{Ti}-\mathrm{C}) v s$ calculated $\Delta \mathrm{H}(\mathrm{H}-\mathrm{C})$. (c) Calculated $\Delta \mathrm{H}(\mathrm{Ti}-\mathrm{C}) v$ s experimental $\Delta \mathrm{H}(\mathrm{Rh}-\mathrm{C})$.
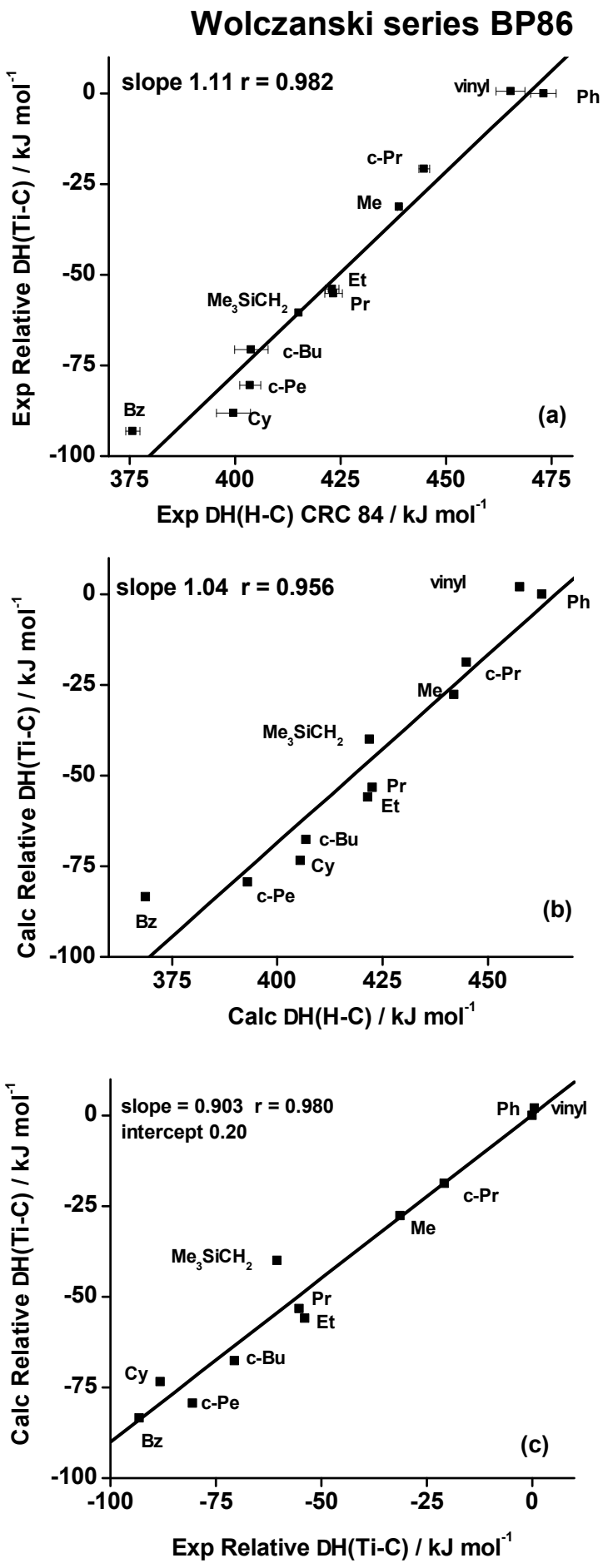
Table S1. Selected geometrical parameters (distances in $\AA$ and angles in degrees) from the crystal structures of the chloride complexes $\left.\mathrm{Rh}(\mathrm{Cl})(\mathrm{R})(\mathrm{Tp})^{\prime}\right)\left(\mathrm{CNCH}_{2} \mathrm{CMe}_{3}\right)$ and the DFT (B3PW91) optimized structures of $\mathrm{Rh}(\mathrm{Cl})(\mathrm{Tp})\left(\mathrm{CNCH}_{3}\right) . \mathrm{N}(1)$ is trans to $\mathrm{R}, \mathrm{N}(2)$ is trans to $\mathrm{CNCH}_{3}$ and $\mathrm{N}(3)$ is trans to $\mathrm{Cl}$. $\alpha=\angle \mathrm{R}-\mathrm{Rh}-\mathrm{N}(2), \beta=\angle \mathrm{R}-\mathrm{Rh}-\mathrm{N}(3), \gamma=\angle \mathrm{R}-\mathrm{Rh}-\mathrm{C}(\mathrm{CNR}), \delta=\angle$ $\mathrm{R}-\mathrm{Rh}-\mathrm{Cl}$.

\begin{tabular}{lccccccccccc}
\hline $\mathrm{R}$ & & $\mathrm{Rh}-\mathrm{R}$ & $\mathrm{Rh}-\mathrm{N}(1)$ & $\mathrm{Rh}-\mathrm{N}(2)$ & $\alpha$ & $\mathrm{Rh}-\mathrm{N}(3)$ & $\beta$ & $\mathrm{Rh}-\mathrm{C}$ & $\gamma$ & $\mathrm{Rh}-\mathrm{Cl}$ & $\delta$ \\
\hline $\mathrm{Et}$ & $\exp ^{a}$ & $2.058(6)$ & $2.146(4)$ & $2.210(5)$ & $90.0(2)$ & $2.051(4)$ & $93.6(2)$ & $2.001(6)$ & $91.5(2)$ & $2.352(2)$ & $87.0(2)$ \\
$\mathrm{Et}$ & $\mathrm{DFT}$ & 2.073 & 2.218 & 2.093 & 88.9 & 2.051 & 91.0 & 1.892 & 93.3 & 2.365 & 89.1 \\
$i$-Pr & $\exp ^{b}$ & $2.110(5)$ & $2.250(4)$ & $2.129(4)$ & $89.2(2)$ & $2.047(4)$ & $95.5(2)$ & $1.891(7)$ & $92.1(1)$ & $2.353(2)$ & $87.5(2)$ \\
$i$-Pr & $\mathrm{DFT}$ & 2.115 & 2.227 & 2.099 & 95.6 & 2.060 & 88.1 & 1.890 & 87.3 & 2.367 & 95.9 \\
$t$-Bu-vinyl & $\exp ^{c}$ & $2.021(6)$ & $2.209(5)$ & $2.092(4)$ & $89.3(2)$ & $2.045(4)$ & $88.3(2)$ & $1.910(6)$ & $90.9(2)$ & $2.352(2)$ & $92.4(2)$ \\
$t$-Bu-vinyl & $\mathrm{DFT}$ & 2.020 & 2.196 & 2.084 & 90.0 & 2.056 & 89.2 & 1.897 & 89.4 & 2.361 & 93.0 \\
$\mathrm{Me}-\mathrm{vinyl}$ & $\mathrm{DFT}$ & 2.018 & 2.196 & 2.083 & 89.9 & 2.056 & 89.4 & 1.897 & 89.0 & 2.361 & 93.0 \\
vinyl & $\mathrm{DFT}$ & 2.014 & 2.196 & 2.083 & 89.9 & 2.055 & 89.3 & 1.898 & 89.3 & 2.360 & 93.0 \\
$c$-Pr & $\exp ^{b}$ & $2.050(9)$ & $2.177(7)$ & $2.088(7)$ & $90.4(3)$ & $2.044(8)$ & $90.9(4)$ & $1.891(1)$ & $92.1(4)$ & $2.349(3)$ & $91.5(3)$ \\
$c-\mathrm{Pr}$ & $\mathrm{DFT}$ & 2.063 & 2.201 & 2.092 & 90.9 & 2.053 & 89.5 & 1.894 & 89.5 & 2.367 & 92.7 \\
$\mathrm{Pe}$ & $\exp ^{d}$ & $2.105(4)$ & $2.226(4)$ & $2.100(4)$ & $92.8(2)$ & $2.064(3)$ & $93.2(1)$ & $1.906(5)$ & $88.7(2)$ & $2.362(1)$ & $87.2(1)$ \\
$\mathrm{Pe}$ & $\mathrm{DFT}$ & 2.073 & 2.216 & 2.091 & 89.0 & 2.052 & 90.9 & 1.893 & 93.3 & 2.364 & 89.4 \\
\hline
\end{tabular}

$a:$ Northcutt, T. O.; Wick, D. D.; Vetter, A. J.; Jones, W. D. J. Am. Chem. Soc. 2001, 123, 7257.

$b:$ Wick, D. D.; Jones, W. D. Inorg. Chem. 1997, 36, 2723.

$c$ Wick, D. D.; Jones, W. D. Organometallics 1999, 18, 495.

$d$ Jones, W. D.; Hessel, T. E. J. Am. Chem. Soc. 1993, 115, 554. 


\section{Cartesian coordinates for the organic molecules and enthalpies (au) at B3PW91}

Energy for $\mathrm{H}^{\bullet}:-0.502176935555$ au.

$\mathrm{Ph}-\mathrm{H}: \mathrm{H}=-232.057926$

$C, 0.2516472442,-0.6970453284,1.1811331975$ C, $0.2521119082,0.6973497457,1.1807306801$ C, $0.000457151,1.3944431163,-0.0003247306$ C, $-0.2516441469,0.6970456887,-1.1811338445$ $C,-0.2520893897,-0.6973497936,-1.1807363022$ C, $-0.0004757252,-1.3944428194,0.0003277597$ $\mathrm{H}, 0.4476921562,-1.2399467461,2.1012792103$ $\mathrm{H}, 0.4485024433,1.2404833478,2.1006856659$ $\mathrm{H}, 0.0008450195,2.4806400787,-0.0003914078$ $\mathrm{H},-0.4477036883,1.2399491148,-2.1012696541$ $\mathrm{H},-0.4484902613,-1.2404841257,-2.1006794896$ $\mathrm{H},-0.0008859347,-2.4806453258,0.0003946924$

$$
\mathrm{Ph}^{\bullet}: \mathrm{H}=-231.382387
$$

C, $1.2249919898,-0.7703800994,0$. C, $0.0000000516,-1.397297122,0$. C, $-1.2249920102,-0.7703791445,0$. $C_{,}-1.212337036,0.6313478557,0$. C, $-0.0000000488,1.322146878,0$. C, $1.212336964,0.6313479004,0$. $\mathrm{H}, 2.160617,-1.3228220821,0$. $\mathrm{H},-2.160616,-1.3228221618,0$. $\mathrm{H},-2.1520910461,1.1782698383,0$. $\mathrm{H},-0.0000000889,2.408386878,0$. $\mathrm{H}, 2.1520909539,1.1782699177,0$.

\section{$\mathrm{H}_{2} \mathrm{C}=\mathrm{CH}-\mathrm{H}: \mathrm{H}=-78.504841$}

$C,-0.0030297804,-0.0000033652,-0.6649067164$ C, $0.0029212429,0.0000033642,0.6650908272$ $\mathrm{H}, 0.9188568477,0.0000102991,-1.2411254604$ $\mathrm{H},-0.9292768846,-0.0000228103,-1.2339009988$ $\mathrm{H},-0.9190198838,-0.0000103034,1.2408463972$ $\mathrm{H}, 0.930091146,0.0000228208,1.2330753971$

\section{$\mathrm{H}_{2} \mathrm{C}=\mathrm{CH} \bullet: \mathrm{H}=-77.831867$}

$C, 0.0829656526,0.5834763831,-0.0048385071$ C, $0.0068330349,-0.7241841455,-0.0003984985$ $\mathrm{H},-0.8081706016,1.2168692168,0.0471320244$ $\mathrm{H}, 1.0370330491,1.1067947434,-0.0604791449$ $\mathrm{H},-0.767654572,-1.4794173863,0.0447691539$

Me-H : H = -40.459202

C, $0 ., 0 ., 0$.

$\mathrm{H}, 0.8913263347,-0.000000438,0.6302629559$ $\mathrm{H},-0.7172836125,0.7277654744,0.3841297462$ $\mathrm{H}, 0.2768615328,0.2663800507,-1.0218039874$ $\mathrm{H},-0.450904255,-0.9941450871,0.0074112854$

$$
\mathrm{Me}^{\bullet}: \mathrm{H}=-39.793024
$$

$C,-0.000034234,0.0000240636,0.0000778859$ $\mathrm{H}, 0.6893106597,0.8330235889,0.0454091163$ $\mathrm{H},-0.9693563781,0.1245120615,-0.4647412528$ $\mathrm{H}, 0.2802511225,-0.9576800318,0.4188648208$

Et-H : H = -79.728941
$C,-0.0001812438,-0.0001234317,-0.7623969556$ C, $0.0001812438,0.0001234317,0.7623969556$ $\mathrm{H}, 1.0188746786,-0.0002184261,-1.1627324099$ $\mathrm{H},-0.509925099,-0.8827912366,-1.1621466627$ $\mathrm{H},-0.5098729678,0.8824450035,-1.1624324823$ $\mathrm{H},-1.0188746786,0.0002184261,1.1627324099$ $\mathrm{H}, 0.5098729678,-0.8824450035,1.1624324823$ $\mathrm{H}, 0.509925099,0.8827912366,1.1621466627$

$$
\mathrm{Et}^{\bullet}: \mathrm{H}=-79.070352
$$

$C, 0.3104851711,-0.093150215,-0.6118781079$ C, $-0.3645803324,0.0855711921,0.6995059319$ $\mathrm{H},-0.4082621163,-0.273315676,-1.4189751945$ $\mathrm{H}, 0.8903962463,0.7984423444,-0.9052889232$ $\mathrm{H}, 1.0177386815,-0.9298012654,-0.5954380228$ $\mathrm{H}, 0.1584527982,-0.1183965377,1.6278524567$ $\mathrm{H},-1.3337539151,0.5685449374,0.7660831596$

$$
\operatorname{Pr}-\mathrm{H}: \mathrm{H}=-119.001195
$$

C, $-0.9927656537,-0.4990278136,-0.6720669978$ C, $0.2713066171,-0.4839423787,0.1836762228$ $\mathrm{H},-1.3189277717,-1.5206127902,-0.8928515098$ $\mathrm{H},-1.8195334438,0.0115622968,-0.1652768784$ $\mathrm{H},-0.8293459303,0.0115356437,-1.6279448219$ C, $0.7524170401,0.9277489449,0.5093495307$ $\mathrm{H}, 0.0875856037,-1.0318348444,1.1167484651$ $\mathrm{H}, 1.069358769,-1.031861271,-0.3334901182$ $\mathrm{H}, 1.659005143,0.9140007814,1.1230859497$ $\mathrm{H}, 0.9781485616,1.4892555073,-0.4043457824$ $\mathrm{H},-0.0120389519,1.4892821604,1.0583221611$

$$
\operatorname{Pr}^{\bullet}: H=-118.342162
$$

$C,-1.2908275469,-0.3078347973,0.1373225188$ $\mathrm{C},-0.0783798578,0.5516116766,0.0602200274$ $\mathrm{H},-2.2823026848,0.1082534332,-0.0097849589$ $\mathrm{H},-1.2225300459,-1.3301427543,0.497919922$ C, $1.2115802007,-0.2298031821,-0.1954359922$ $\mathrm{H},-0.2133497879,1.3144640843,-0.7192741475$ $\mathrm{H}, 0.0357840783,1.1260880819,0.9980202999$ $\mathrm{H}, 2.0819117971,0.4334641782,-0.2195383845$ $\mathrm{H}, 1.3811826147,-0.9737196787,0.5907556646$ $\mathrm{H}, 1.1650672523,-0.7622495278,-1.1507377193$

$$
i-\operatorname{Pr}-\mathrm{H}: \mathrm{H}=-119.001204
$$

$C,-0.2797576442,-1.1990045383,0.4133134304$ C, $-0.271331635,0.3276595803,0.4007792725$ $\mathrm{H},-1.3038994221,0.6983214514,0.3629740662$ $\mathrm{H},-0.8525136041,-1.5926366754,1.2593836856$ $\mathrm{H},-0.7249837759,-1.5994680288,-0.5046309672$ $\mathrm{H}, 0.7379115731,-1.5994120422,0.4857170598$ C, $0.5201247957,0.9087373664,-0.7683555991$ $\mathrm{H}, 0.147305196,0.6983786054,1.3454095261$ $\mathrm{H}, 0.512230871,2.0035583609,-0.7567629352$ $\mathrm{H}, 1.5663142635,0.5834818201,-0.7380815782$ $\mathrm{H}, 0.1034197364,0.5834202806,-1.7284314233$

$i$-Pr• : H = -118.348891 
$C, 0.0111762019,-0.1970636819,1.2969825294$ C, $0.0111763696,0.5334762164,-0.0000000887$ $\mathrm{H},-0.2352075946,1.5925008052,-0.0000002996$ $\mathrm{H}, 0.2510746546,0.4609932216,2.1381689882$ $\mathrm{H},-0.9697816897,-0.6530497484,1.5189706863$ $\mathrm{H}, 0.735722863,-1.0222382781,1.2948368724$ C, $0.0111765373,-0.1970641203,-1.2969824599$ $\mathrm{H}, 0.2510752074,0.4609924988,-2.1381690791$ $\mathrm{H}, 0.7357231978,-1.0222387158,-1.2948363366$ $\mathrm{H},-0.969781297,-0.6530502619,-1.5189707163$

\section{$t-\mathrm{Bu}-\mathrm{H}: \mathrm{H}=-158.274818$}

C, $0.7069015673,-1.0141794205,0.776134196$ C, $-0.0194434227,0.2396298812,0.2846966392$ $\mathrm{H},-0.0768630974,0.9467933935,1.1255432355$ $\mathrm{H}, 1.7209136735,-0.7811947015,1.1190811948$ $\mathrm{H}, 0.1727733039,-1.4871181377,1.6075104384$ $\mathrm{H}, 0.7912734864,-1.75563487,-0.0280060424$ C, $-1.4493772037,-0.0880483801,-0.1498041842$ C, $0.7573956817,0.918072678,-0.8452864754$ $\mathrm{H},-1.9847123032,0.810544314,-0.475407392$ $\mathrm{H},-1.44915864,-0.7960044934,-0.9878264179$ $\mathrm{H},-2.0219464654,-0.5416191855,0.666569263$ $\mathrm{H}, 0.8456387541,0.2516400769,-1.7121599882$ $\mathrm{H}, 0.2578158209,1.8335289037,-1.1805120357$ $\mathrm{H}, 1.7714057325,1.1862161481,-0.5292333093$

\section{$t-\mathrm{Bu}^{\bullet}: \mathrm{H}=-157.627428$}

C, $0.21885,-0.38196,-1.41842$ C, $0.06829,-0.11988,0.04433$ $\mathrm{H}, 1.27296,-0.44384,-1.71336$ $\mathrm{H},-0.27393,-1.31449,-1.71717$ $\mathrm{H},-0.23024,0.41935,-2.03293$ C, $-1.23596,-0.42996,0.70304$ $\mathrm{C}, 0.99612,0.8485,0.70187$ $\mathrm{H},-1.1316,-0.51401,1.7911$ $\mathrm{H},-1.99076,0.35668,0.52168$ $\mathrm{H},-1.66798,-1.36552,0.32897$ $\mathrm{H}, 0.70079,1.89756,0.51838$ $\mathrm{H}, 1.01525,0.71805,1.79017$ $\mathrm{H}, 2.02175,0.74607,0.32827$

\section{$c-\mathrm{Pr}-\mathrm{H}: \mathrm{H}=-117.776278$}

$C_{,}-0.3760096659,-0.7520282351,0.217083464$ $C_{,}-0.3760488109,0.7520086387,0.2171097654$ C, $0.7520970211,0.0000409469,-0.4342154825$ $\mathrm{H},-0.1765477301,-1.2636522574,1.1538658449$ $\mathrm{H},-1.0875469815,-1.2636483742,-0.4240599792$ $\mathrm{H},-0.1762802758,1.2638019106,1.1537339174$ $\mathrm{H},-1.0872936126,1.2638057938,-0.4242163038$ $\mathrm{H}, 0.8081916118,-0.0002157206,-1.5186051912$ $\mathrm{H}, 1.7192455392,-0.000219604,0.0594153362$

\section{$c-\operatorname{Pr}^{\bullet}: \mathrm{H}=-117.107537$}

$C, 0.7730794912,0.3110083513,0.1532261607$ C, $-0.754457038,0.3708726298,0.1053760094$ $C,-0.0266352171,-0.8586109942,-0.2239132534$ $\mathrm{H}, 1.3475745695,0.8347693269,-0.6105616635$ $\mathrm{H}, 1.2361475594,0.3386420366,1.139233111$ $\mathrm{H},-1.2373866022,0.9360741594,-0.6915357187$ $\mathrm{H},-1.2746575944,0.4370404049,1.0605820201$ $\mathrm{H},-0.0236013494,-1.4861458493,-1.1058512493$

\footnotetext{
$c-\mathrm{Bu}-\mathrm{H}: \mathrm{H}=-157.051993$
}

$C,-0.0177412295,0.2364339944,1.0599964228$ C, $-0.012054741,1.0565310922,-0.2518099516$ $C,-0.238695526,-0.263349629,-1.0263916887$ C, $0.268495506,-1.0296159608,0.2182043855$ $\mathrm{H}, 0.6994574343,0.5087352624,1.8403808176$ $\mathrm{H},-1.0176032215,0.2024009487,1.5057522343$ $\mathrm{H}, 0.9789275907,1.475331664,-0.4570800392$ $\mathrm{H},-0.7538999892,1.8531353898,-0.3641000055$ $\mathrm{H}, 0.2958466505,-0.4042748493,-1.9708095906$ $\mathrm{H},-1.3043024205,-0.4461006456,-1.2014752769$ $\mathrm{H}, 1.3429633826,-1.2316232238,0.152806223$ $\mathrm{H},-0.2414134842,-1.9576015267,0.4945306294$

\section{$c-\mathrm{Bu}^{\bullet}: \mathrm{H}=-156.397994$}

C, $0.1040498398,0.0236373466,-1.0360468951$ C, $-0.0827126079,-1.0989995082,0.0280374466$ $C,-0.1073938066,-0.0244572046,1.0715954078$ C, $0.0703839828,1.0962330242,0.0934973666$ $\mathrm{H}, 1.0480453393,-0.0258226731,-1.5850527457$ $\mathrm{H},-0.7121833056,0.1021168407,-1.7589399863$ $\mathrm{H}, 0.7475526871,-1.8179219284,0.0967347442$ $\mathrm{H},-1.0050159706,-1.6900976508,-0.0761836869$ $\mathrm{H},-0.2158433726,-0.0491245847,2.151358007$ $\mathrm{H}, 0.9920546558,1.6876899971,0.2012508959$ $\mathrm{H},-0.760574482,1.8146780511,0.0283328164$

\section{$c-\mathrm{Pe}-\mathrm{H}: \mathrm{H}=-196.354397$}

$C_{,}-0.4785177195,0.8108659546,0.8844607631$ C, $-0.5166719791,-0.7238488569,0.9211417538$ C, $0.6110430769,-1.1246995965,-0.0329779749$ C, $0.4445519287,-0.1455105338,-1.2041407893$ C, $-0.0599031759,1.1733728933,-0.5637650394$ $\mathrm{H},-1.4307843218,1.2655418133,1.1754258814$ $\mathrm{H}, 0.274192945,1.173814712,1.5945812312$ $\mathrm{H},-1.4763439659,-1.0882893012,0.5309657796$ $\mathrm{H},-0.4002274536,-1.1313895963,1.9308716754$ $\mathrm{H}, 1.5813089961,-0.9677768542,0.4569033071$ $\mathrm{H}, 0.5689852436,-2.1742477325,-0.3426279509$ $\mathrm{H}, 1.3667310017,-0.0107952946,-1.7782836306$ $\mathrm{H},-0.3067759723,-0.5354844369,-1.9012799081$ $\mathrm{H}, 0.7200535436,1.9416280117,-0.5633466156$ $\mathrm{H},-0.9001476555,1.5859209368,-1.1315196357$

\section{$c-\mathrm{Pe}^{\bullet}: \mathrm{H}=-195.705027$}

$C, 0.4135099436,-1.2153548145,0.2660505485$ C, $-0.4222667129,-0.7180634215,-0.9299718322$ $C,-0.1940738886,0.8001870363,-0.9385136629$ $C,-0.1427475873,1.1792773868,0.554787044$ C, $0.3983834481,-0.0530821574,1.2077310701$ $\mathrm{H}, 0.0175992724,-2.1398345795,0.7083739477$ $\mathrm{H}, 1.4424127002,-1.4563563896,-0.0558813734$ $\mathrm{H},-1.4828979712,-0.9309495242,-0.7472879603$ $\mathrm{H},-0.1506584242,-1.1979270731,-1.8754868014$ $\mathrm{H}, 0.7728202734,1.0255630337,-1.4053689887$ $\mathrm{H},-0.9615285863,1.3461191887,-1.4961964721$ $\mathrm{H}, 0.4624470346,2.0758713457,0.7469245665$ $\mathrm{H},-1.1549868155,1.4180586487,0.9272357758$ $\mathrm{H}, 0.7379614893,-0.0983288541,2.2371888778$

Cy-H : H = -235.634606 
$C,-1.1220334464,-0.5850210784,-0.7664363841$ C, $-1.1208292892,-0.5892514952,0.7650685216$ C, $0.304867405,-0.5839256932,1.324700431$ C, $1.1219660099,0.5850471541,0.7664965811$ C, $1.1208402445,0.5893411605,-0.7650088399$ C, $-0.3048089302,0.5838040424,-1.3247962621$ $\mathrm{H}, 0.2829563604,-0.5439375351,2.4206602858$ $\mathrm{H},-1.6534701437,0.3017532188,1.1282626647$ $\mathrm{H},-1.6749369612,-1.4570287941,1.1434326766$ $\mathrm{H},-0.6931629158,-1.5303930078,-1.129669914$ $\mathrm{H},-2.1504703639,-0.5455557126,-1.1458712893$ $\mathrm{H}, 0.6929133259,1.5303286601,1.1297492253$ $\mathrm{H}, 2.1503826485,0.5457229291,1.1459998898$ $\mathrm{H}, 1.6748153475,1.4572404816,-1.14328785$ $\mathrm{H}, 1.6536815779,-0.301539019,-1.1282140383$ $\mathrm{H},-0.799980186,1.5290251847,-1.0585355562$ $\mathrm{H},-0.2827269945,0.5434892886,-2.4207407308$ $\mathrm{H}, 0.7999863427,-1.5290702353,1.0580703507$

\section{$\mathrm{Cy}^{\bullet}: \mathrm{H}=-234.981350$}

$C,-1.2177636218,-0.2217641042,-0.8652163939$ $C_{,}-1.3402755965,0.0375461067,0.6002750224$ C, $-0.1658057256,-0.2266495242,1.4838683969$ C, $1.1429458563,0.3017492634,0.8731015718$ $C, 1.2993405135,-0.14625286,-0.5821697136$ C, $0.110224713,0.3065453456,-1.4330264013$ $\mathrm{H},-0.3241484315,0.2001566466,2.4815203483$ $\mathrm{H},-2.3258331106,0.1621820497,1.0418827124$ $\mathrm{H},-1.2619086337,-1.3118113813,-1.0564471351$ $\mathrm{H},-2.0668153764,0.2082498032,-1.4099589878$ $\mathrm{H}, 1.1378342652,1.3994423266,0.9112258029$ $\mathrm{H}, 1.9984963684,-0.0319358991,1.4725749372$ $\mathrm{H}, 2.2343725417,0.2463526773,-1.0000755661$ $\mathrm{H}, 1.37794061,-1.2428831912,-0.6196487744$ $\mathrm{H}, 0.0800823669,1.4043546547,-1.4507973404$ $\mathrm{H}, 0.2322141522,-0.0237330703,-2.4716387096$ $\mathrm{H},-0.0542315858,-1.3174199799,1.6403678187$

\section{$\mathrm{Ph}-\mathrm{CH}_{2}-\mathrm{H}: \mathrm{H}=-271.334953$}

$C, 0.4513865694,1.6465948643,-0.8372493007$ $C_{,}-0.2388504805,1.6397211638,0.3733974184$ $C,-0.5640608312,0.431956564,0.9871408909$ $C,-0.2101583132,-0.7907353009,0.4059326679$ C, $0.4857265607,-0.7685316005,-0.8078534026$ C, $0.8134276583,0.4363846967,-1.4258557152$ $\mathrm{H}, 0.7087071672,2.5873009886,-1.3158996519$ $\mathrm{H},-0.5218379811,2.5773440868,0.8444495685$ $\mathrm{H},-1.0998025041,0.4365025617,1.9338946191$ $\mathrm{H}, 0.7756838766,-1.7082166645,-1.2729331416$ $\mathrm{H}, 1.3564771205,0.4293900667,-2.3672149219$ C, $-0.5916576463,-2.0953037165,1.0553084108$ $\mathrm{H},-0.6465705058,-2.0007162596,2.1438548699$ $\mathrm{H},-1.5748961222,-2.4363799824,0.708380659$ $\mathrm{H}, 0.1273578463,-2.8857448225,0.8205421818$

\section{$\mathrm{Ph}-\mathrm{CH}_{2}^{\bullet}: \mathrm{H}=-270.694983$}

C, $-0.1024940791,0.1027192414,-1.8312521605$ C, $1.1447393117,0.0633373726,-1.1964624706$ C, $1.2279293253,-0.0140146,0.1830848997$ $C, 0.0553868633,-0.0554168125,0.989591925$ C, $-1.1998298885,-0.0140146,0.3189651189$ $C_{,}-1.2711020772,0.0633373726,-1.0612492849$ $\mathrm{H},-0.1630855928,0.1634654624,-2.9138350889$
$\mathrm{H}, 2.0545724962,0.093708344,-1.789971943$ $\mathrm{H}, 2.1992901875,-0.044245466,0.6702699258$ $\mathrm{H},-2.1107594336,-0.044245466,0.911500804$ $\mathrm{H},-2.2414820904,0.093708344,-1.5495243585$ C, $0.1338868097,-0.1345120583,2.3921431515$ $\mathrm{H}, 1.0923922477,-0.1655033566,2.8994214744$ $\mathrm{H},-0.7620254053,-0.1655033566,3.0032121121$

\section{$\mathrm{H}_{2} \mathrm{C}=\mathrm{CH}-\mathrm{CH}_{2}-\mathrm{H}: \mathrm{H}=-117.783549$}

C, $-0.5164136534,-0.0281360063,-1.1926037986$ C, $-0.4528564031,-0.0008843452,0.1383038212$ $\mathrm{H}, 0.3825835817,-0.0331793731,-1.8055935164$ $\mathrm{H},-1.4666307563,-0.046390132,-1.7186672186$ $\mathrm{H},-1.3830089485,0.0030437611,0.7083241005$ C, $0.8101565434,0.0254725431,0.9417618631$ $\mathrm{H}, 0.855900617,0.9180848736,1.5782242489$ $\mathrm{H}, 0.8701198154,-0.8400017817,1.6135447767$ $\mathrm{H}, 1.6957167683,0.0197295312,0.2993962943$

\section{$\mathrm{H}_{2} \mathrm{C}=\mathrm{CH}-\mathrm{CH}^{\bullet}: \mathrm{H}=-117.148401$}

C, $0.2023192778,-0.1711702092,-1.215544985$ C, $-0.4421413268,0.0293005374,-0.0061771169$ $\mathrm{H}, 1.2847214848,-0.2514361239,-1.2674957307$ $\mathrm{H},-0.3488561601,-0.2542995567,-2.1459855541$ $\mathrm{H},-1.5299341151,0.1014022976,-0.0214978834$ C, $0.1890566857,0.1452270362,1.2210035528$ $\mathrm{H},-0.3720568099,0.3020085063,2.1358896089$ $\mathrm{H}, 1.2707177806,0.0821806903,1.3034008537$

\section{$\mathrm{H}_{2} \mathrm{C}=\mathrm{C}(\mathrm{Me})-\mathrm{CH}_{2}-\mathrm{H}: \mathrm{H}=-157.061947$}

C, $-0.108651767,1.454018935,0.0001817881$ $C,-0.0090622526,0.1213244516,-0.0000173727$ $\mathrm{H},-1.0722211985,1.9534098997,-0.0602130374$ $\mathrm{H}, 0.7699973299,2.0910527093,0.0607603962$ $C,-1.2173635589,-0.7705131495,-0.0834097026$ $\mathrm{H},-1.1636359081,-1.4235381533,-0.9641285451$ $\mathrm{H},-1.2783464938,-1.4321815426,0.7903623403$ $\mathrm{H},-2.1453995463,-0.1957276213,-0.1412518051$ C, $1.3183459932,-0.5810377088,0.0832419638$ $\mathrm{H}, 1.4770302644,-1.226180735,-0.7905931115$ $\mathrm{H}, 2.1506485684,0.1252955251,0.1411805949$ $\mathrm{H}, 1.3623167163,-1.2348852351,0.9638997428$

\section{$\mathrm{H}_{2} \mathrm{C}=\mathrm{C}(\mathrm{Me})-\mathrm{CH}^{\bullet}: \mathrm{H}=-156.424031$}

C, $0.9313328328,0.0625050139,1.0909136137$ C, $0.0063666821,0.0724312736,0.0543822763$ $\mathrm{H}, 1.5034005588,-0.8272047617,1.3345011538$ $\mathrm{H}, 1.1099834797,0.9502858799,1.6900742223$ C, $-0.1846184011,-1.175433134,-0.7814446068$ $\mathrm{H},-1.2026168462,-1.2412636969,-1.1758593473$ $\mathrm{H}, 0.5004382908,-1.1775609155,-1.637150984$ $\mathrm{H}, 0.0131672433,-2.0807046805,-0.2004312831$ C, $-0.7309140083,1.2113620249,-0.2444429368$ $\mathrm{H},-1.4613815504,1.2219333146,-1.0472349009$ $\mathrm{H},-0.5959938093,2.1293237902,0.319651061$

\section{$\mathrm{Me}_{3} \mathrm{SiCH}_{2}-\mathrm{H}: \mathrm{H}=-163.394326$}

Si, $0 ., 0 ., 0$. C, $1.5495662677,0 ., 1.0952491324$ C, $-1.5495662677,0 ., 1.0952491324$ C, $0 .,-1.5495662677,-1.0952491324$ C, $0 ., 1.5495662677,-1.0952491324$ $\mathrm{H}, 2.4616656192,0 ., 0.4889686916$ $\mathrm{H}, 1.5781693269,0.8844627485,1.7405395304$ $\mathrm{H}, 1.5781693269,-0.8844627485,1.7405395304$ 
$\mathrm{H},-2.4616656192,0 ., 0.4889686916$

$\mathrm{H},-1.5781693269,-0.8844627485,1.7405395304$ $\mathrm{H},-1.5781693269,0.8844627485,1.7405395304$ $\mathrm{H}, 0 .,-2.4616656192,-0.4889686916$ $\mathrm{H},-0.8844627485,-1.5781693269,-1.7405395304$ $\mathrm{H}, 0.8844627485,-1.5781693269,-1.7405395304$ $\mathrm{H}, 0 ., 2.4616656192,-0.4889686916$ $\mathrm{H}, 0.8844627485,1.5781693269,-1.7405395304$ $\mathrm{H},-0.8844627485,1.5781693269,-1.7405395304$

\section{$\mathrm{Me}_{3} \mathrm{SiCH}_{2}^{\bullet}: \mathrm{H}=-162.735424$}

\section{Si, $0 ., 0.0364630398,-0.025010784$} C, $1.555323925,0.0892396163,1.0591899167$ C, $-1.555323925,0.0892396163,1.0591899167$ C, $0 .,-1.5753732839,-1.0340783545$ C, $0 ., 1.5116524705,-1.1724282875$ $\mathrm{H}, 2.4632728812,0.0875622982,0.4469387012$ $\mathrm{H}, 1.5714268157,0.9895778426,1.6815494188$ $\mathrm{H}, 1.5993842842,-0.7804074103,1.7234984442$ $\mathrm{H},-2.4632728812,0.0875622982,0.4469387012$ $\mathrm{H},-1.5993842842,-0.7804074103,1.7234984442$ $\mathrm{H},-1.5714268157,0.9895778426,1.6815494188$ $\mathrm{H}, 0 .,-2.4472290111,-0.3703615648$ $\mathrm{H},-0.8849178011,-1.6392573772,-1.6752039779$ $\mathrm{H}, 0.8849178011,-1.6392573772,-1.6752039779$ $\mathrm{H}, 0.9134895995,1.966622616,-1.5521458901$ $\mathrm{H},-0.9134895995,1.966622616,-1.5521458901$

\section{$(\mathrm{Me})(\mathrm{H}) \mathrm{C}=\mathrm{CH}-\mathrm{H}: \mathrm{H}=-117.783549$}

$C,-0.5164136534,-0.0281360063,-1.1926037986$ $C,-0.4528564031,-0.0008843452,0.1383038212$ $\mathrm{H}, 0.3825835817,-0.0331793731,-1.8055935164$ $\mathrm{H},-1.4666307563,-0.046390132,-1.7186672186$ $\mathrm{H},-1.3830089485,0.0030437611,0.7083241005$ C, $0.8101565434,0.0254725431,0.9417618631$ $\mathrm{H}, 0.855900617,0.9180848736,1.5782242489$ $\mathrm{H}, 0.8701198154,-0.8400017817,1.6135447767$ $\mathrm{H}, 1.6957167683,0.0197295312,0.2993962943$

\section{$(\mathrm{Me})(\mathrm{H}) \mathrm{C}=\mathrm{CH}^{\bullet}: \mathrm{H}=-117.108565$}

$C,-0.0140580546,1.3549127005,0.2345622597$ C, $0.0273403672,0.2371548773,-0.4543633512$ $\mathrm{H},-0.0767324293,1.6447793105,1.2766346759$ $\mathrm{H}, 0.0928877456,0.2940118037,-1.544004185$ $\mathrm{C},-0.0078107359,-1.1548878911,0.1291168391$ $\mathrm{H},-0.868286007,-1.7140432991,-0.2571107532$ $\mathrm{H}, 0.8927448889,-1.714088982,-0.1511293951$ $\mathrm{H},-0.0734436187,-1.1337369528,1.2197151737$

\section{$(t-\mathrm{Bu})(\mathrm{H}) \mathrm{C}=\mathrm{CH}-\mathrm{H}: \mathrm{H}=-235.600656$}

C, $0.3774865422,0.1871080385,2.163014421$ $\mathrm{H},-0.1044902691,1.1571298556,2.2479792248$ C, $0.4642211953,-0.471621513,1.0076981339$ $\mathrm{H}, 0.9642579746,-1.4430183201,1.0024224065$ C, $-0.0553875914,-0.0399892308,-0.3469195543$ C, $-1.0519248262,-1.1059198536,-0.8417238401$ $\mathrm{H},-0.5844549438,-2.0960333624,-0.8867931908$ $\mathrm{H},-1.9179041895,-1.1736874636,-0.1751823667$ $\mathrm{H},-1.4127486684,-0.8608225711,-1.8473713499$ C, $-0.748905869,1.32442209,-0.2986457293$ $\mathrm{H},-1.1107751081,1.6026266311,-1.2943189161$ $\mathrm{H},-1.6101531258,1.3110174048,0.3777250322$ $\mathrm{H},-0.0634708465,2.1091810242,0.0385995828$
$C, 1.136505828,0.0234087386,-1.3213335381$ $\mathrm{H}, 1.863757634,0.7778544402,-1.00398866$ $\mathrm{H}, 1.6551291448,-0.9403080686,-1.3775827808$ $\mathrm{H}, 0.7968959283,0.2794227809,-2.3316382077$ $\mathrm{H}, 0.7919847954,-0.2278119702,3.0776098668$

\section{$(t-\mathrm{Bu})(\mathrm{H}) \mathrm{C}=\mathrm{CH}^{\bullet}: \mathrm{H}=-234.927152$}

C, $0.3777002043,0.2075851737,2.2094589797$ $\mathrm{H},-0.0279186049,1.1448646318,2.5667573632$ C, $0.4760560581,-0.4603588526,1.0841084886$ $\mathrm{H}, 0.9797647436,-1.4326893473,1.0965852302$ C, $-0.0404365299,-0.0471485689,-0.2954256713$ $C,-1.0307675416,-1.1225789381,-0.7744364297$ $\mathrm{H},-0.5594495394,-2.1112299727,-0.8057375148$ $\mathrm{H},-1.897370945,-1.1838796424,-0.108374158$ $\mathrm{H},-1.3906821412,-0.891246773,-1.7837628652$ $C,-0.7350846002,1.3162599237,-0.2535566015$ $\mathrm{H},-1.0962756043,1.5910950794,-1.2504882061$ $\mathrm{H},-1.5954966053,1.302448148,0.4237050467$ $\mathrm{H},-0.0493260861,2.1006605752,0.083552824$ C, $1.1600828863,0.0084189665,-1.2558219775$ $\mathrm{H}, 1.8826488995,0.7672026201,-0.9387311932$ $\mathrm{H}, 1.6801606229,-0.9550546397,-1.298142634$ $\mathrm{H}, 0.8286423981,0.2547630946,-2.2713246227$

\section{Mesityl-H : $\mathrm{H}=-349.888816$}

C, $0.3595633152,1.0781670065,2.683984961$ $\mathrm{H}, 1.2015505176,1.7784830216,2.7234033176$ $\mathrm{H},-0.5331455317,1.6070486418,3.0299213611$ C, $0.1691773772,0.5265433726,1.2948021285$ $C, 1.2384382121,-0.0788895128,0.6206500187$ C, $1.0855524125,-0.6066905359,-0.6603515599$ C, $-0.1717535171,-0.5200497517,-1.2742725576$ $C,-1.2549317353,0.0785122316,-0.633389774$ C, $-1.0669448832,0.5972079367,0.6551427223$ $\mathrm{H}, 2.2098906666,-0.1392534277,1.1086658597$ $\mathrm{H},-0.3042922298,-0.9280746404,-2.2750863774$ $\mathrm{H},-1.9058470762,1.0657842257,1.1673435354$ C, $-2.5978208302,0.1849055156,-1.3086018942$ $\mathrm{H},-2.8284252108,1.2241686263,-1.5723070064$ $\mathrm{H},-2.6314895377,-0.4045309798,-2.2291140296$ $\mathrm{H},-3.4030687265,-0.1663993271,-0.6544249391$ C, $2.2385615598,-1.2610868208,-1.3766685458$ $\mathrm{H}, 2.4400963364,-0.7728214747,-2.3368334214$ $\mathrm{H}, 3.1552602684,-1.2192177552,-0.7820393771$ $\mathrm{H}, 2.0279718565,-2.3153831822,-1.5908589615$ $\mathrm{H}, 0.5724472014,0.2784796215,3.4035570449$

\section{Mesityl $^{\bullet}: \mathrm{H}=-349.249110$}

C, $0.3446206018,1.1207448027,2.6417316143$ $\mathrm{H}, 1.3083921391,1.0980517967,3.1395419898$ $\mathrm{H},-0.4853270876,1.5786113323,3.1696848973$ C, $0.176964709,0.575553201,1.3566619887$ C, $1.2637187275,-0.0251045625,0.6629295403$ C, $1.1081038416,-0.5658384524,-0.6056102528$ C, $-0.1580994371,-0.5141753302,-1.2120060822$ $C,-1.2609183592,0.0688659364,-0.5658398368$ $C,-1.0856365221,0.6043178095,0.7023685634$ $\mathrm{H}, 2.2377624976,-0.0569619466,1.1466223304$ $\mathrm{H},-0.2879132515,-0.9363215608,-2.2071090859$ $\mathrm{H},-1.929509522,1.0595237334,1.2165679368$ $\mathrm{C},-2.6038957281,0.1056124413,-1.2484222521$ $\mathrm{H},-2.5532042702,0.654843289,-2.1954814873$ 
$\mathrm{H},-2.9589016933,-0.9047980219,-1.4812088819$

$\mathrm{H},-3.3583799381,0.5888304634,-0.6218255384$

C, $2.2675316155,-1.1995689592,-1.3301559631$

$\mathrm{H}, 2.4682248674,-0.6909364338,-2.2799770881$

$\mathrm{H}, 3.1818297974,-1.1629850759,-0.7317503213$

$\mathrm{H}, 2.0626897661,-2.2502988952,-1.5650086704$

\section{$\mathrm{Pe}-\mathrm{H}: \mathrm{H}=-197.545600$}

C, $-0.2607989564,-0.0000013616,-0.176128448$ $C_{r}-0.2836843225,0.0000153046,1.3528308181$

$\mathrm{H},-0.8105583581,-0.8772531334,-0.5471671524$ $\mathrm{H},-0.8105524484,0.87724609,-0.5471860559$ C, $-1.695341263,0.000026048,1.9353362235$ $\mathrm{H}, 0.2657373096,-0.8767644027,1.7222484672$ $\mathrm{H}, 0.2657427775,0.8767995834,1.72222949$ $\mathrm{H},-1.679185079,0.0000380268,3.0301583034$ $\mathrm{H},-2.2575295801,0.8834058689,1.61173968$ $\mathrm{H},-2.2575349912,-0.8833574202,1.6117590604$ C, $1.1484573002,-0.0000124881,-0.7694550321$ $\mathrm{H}, 1.6967916917,0.8768353527,-0.3981874546$ $\mathrm{H}, 1.6967850795,-0.8768572104,-0.3981703106$ C, $1.1598758856,-0.0000273445,-2.2963902516$
$\mathrm{H}, 0.6491060078,-0.8835661645,-2.6963092343$ $\mathrm{H}, 0.6491123218,0.8835073125,-2.6963264913$ $\mathrm{H}, 2.1810334056,-0.0000348534,-2.6919481611$

\section{$\mathrm{Pe}^{\bullet}: \mathrm{H}=-196.886704$}

$C, 2.6005091031,-0.2133422409,-0.1733053254$ C, $1.3297612508,0.5490027003,-0.0382300358$ $\mathrm{H}, 3.5500294097,0.2245639962,0.1173419361$ $\mathrm{H}, 2.6246278046,-1.1602939171,-0.7048449437$ $\mathrm{C}, 0.0773640702,-0.333612478,-0.008627791$ $\mathrm{H}, 1.3633122666,1.1733291953,0.8667967736$ $\mathrm{H}, 1.2295780343,1.2698107138,-0.8721014447$ $\mathrm{H}, 0.0532027568,-0.9525410041,-0.9168449763$ $\mathrm{H}, 0.1510733062,-1.0344793101,0.8338953373$ C, $-1.224694367,0.4598273034,0.0982417902$ $\mathrm{H},-1.2910899221,1.1611881656,-0.7451351143$ $\mathrm{H},-1.1968877504,1.0804753763,1.0044956996$ C, $-2.4681445632,-0.4259272949,0.1252617136$ $\mathrm{H},-3.3844345305,0.1689176057,0.2005438039$ $\mathrm{H},-2.5411013164,-1.0342242151,-0.7835795125$ $\mathrm{H},-2.4470830227,-1.1124345464,0.9793903316$ 


\section{Cartesian coordinates for the organic molecules and enthalpies (au) at BP86}

Energy for $\mathrm{H}^{\bullet}:-0.498103409968 \mathrm{au}$.

$\mathrm{Ph}-\mathrm{H}: \mathrm{H}=-232.138877$

$C_{,}-1.2165996569,0.0005551985,-0.7025030951$ $\mathrm{C},-1.2166367092,0.0010015581,0.7023011799$ $C,-0.0000674732,0.0004478839,1.4047294358$ C, $1.2165996428,-0.0005553254,0.7025030338$ C, $1.2166367926,-0.0010028408,-0.7023011712$ C, $0.0000674152,-0.0004467838,-1.4047293741$ $\mathrm{H},-2.1651759633,0.000987451,-1.2501677978$ $\mathrm{H},-2.1652099139,0.0017845814,1.2499775236$ $\mathrm{H},-0.0001651657,0.0007967718,2.5000525157$ $\mathrm{H}, 2.1651756903,-0.0009869143,1.2501682214$ $\mathrm{H}, 2.1652096494,-0.0017855036,-1.2499780209$ $\mathrm{H}, 0.000165634,-0.0007945291,-2.5000524968$

$\mathrm{Ph}^{\bullet}: \mathrm{H}=-231.464639$

C, $1.2332687755,-0.7770841622,0$. C, $0.0000000259,-1.4069684095,0$. C, $-1.2332688127,-0.7770840481,0$. C, $-1.2212624626,0.6365261035,0$. C, $0.0000000252,1.3321070017,0$. C, $1.2212624186,0.6365261362,0$. $\mathrm{H}, 2.17761703,-1.3321815311,0$. $\mathrm{H},-2.177616877,-1.3321817486,0$. $\mathrm{H},-2.1695251626,1.1866128607,0$. $\mathrm{H}, 0.0000000106,2.42700191,0$. $\mathrm{H}, 2.1695251795,1.1866127786,0$.

$\mathrm{H}_{2} \mathrm{C}=\mathrm{CH}-\mathrm{H}: \mathrm{H}=-78.529844$

$C,-0.0028722352,-0.0000033878,-0.6700088942$ C, $0.002878347,0.0000033879,0.6700097382$ $\mathrm{H}, 0.9256356686,0.0000103693,-1.2514424077$ $\mathrm{H},-0.9362774042,-0.0000229836,-1.2434823273$ $\mathrm{H},-0.9256558237,-0.0000103699,1.2513873916$ $\mathrm{H}, 0.9362608883,0.0000229835,1.2435322796$

\section{$\mathrm{H}_{2} \mathrm{C}=\mathrm{CH} \bullet: \mathrm{H}=-77.857569$}

C, $0.0843027422,0 ., 0.5868615684$

C, $0.0053290384,0 .,-0.7287936492$

$\mathrm{H},-0.8133913603,0 ., 1.230621317$

$\mathrm{H}, 1.0474562655,0.1 .1157364078$

$\mathrm{H},-0.7718555892,0 .,-1.4947652399$

Me-H : H $=-40.467775$

$\mathrm{C}, 0 ., 0 ., 0$.

$\mathrm{H},-0.0000001794,-0.0000000041,1.099877082$ $\mathrm{H}, 1.0369741173,-0.0000003623,-0.3666255248$ $\mathrm{H},-0.5184872839,-0.8980456936,-0.3666257819$ $\mathrm{H},-0.518486654,0.8980460601,-0.3666257752$

\section{$\mathrm{Me}^{\bullet}: \mathrm{H}=-39.801438$}

C, $0.0000399823,0.0000692515,0.0000282718$ $\mathrm{H},-0.1818335412,-0.3149446192,1.028096016$ $\mathrm{H}, 0.9086872309,-0.3149445999,-0.5141326517$ $\mathrm{H},-0.7270935837,0.6294737103,-0.5141329951$

Et-H : H = -79.749381
$C,-0.0001755925,-0.0001242312,-0.7673288167$ C, $0.0001755925,0.0001242312,0.7673288167$ $\mathrm{H}, 1.0268021402,-0.0002200397,-1.1713078073$ $\mathrm{H},-0.5138917952,-0.889651046,-1.1707109148$ $\mathrm{H},-0.5138392426,0.8893022705,-1.1709989574$ $\mathrm{H},-1.0268021402,0.0002200397,1.1713078073$ $\mathrm{H}, 0.5138392426,-0.8893022705,1.1709989574$ $\mathrm{H}, 0.5138917952,0.889651046,1.1707109148$

$\mathrm{Et}^{\bullet}: \mathrm{H}=-79.090847$

$C, 0.0881103767,-0.6868289669,0.0507541713$ C, $-0.0810229334,0.7911996453,-0.0466436735$ $\mathrm{H}, 0.1168213213,-1.0319797718,1.0997414354$ $\mathrm{H},-0.7488208172,-1.2406404225,-0.4325793866$ $\mathrm{H}, 1.0109979314,-1.0317091286,-0.4489100318$ $\mathrm{H}, 0.2563993146,1.339192713,-0.9305604161$ $\mathrm{H},-0.6779224099,1.3389125394,0.6876454124$

$\operatorname{Pr}-\mathrm{H}: \mathrm{H}=-119.032999$

C, $0.502284073,-0.0000206817,-1.2065954305$ C, $0.4873489795,0.0000051951,0.3299343337$ $\mathrm{H}, 1.5315445644,-0.0000281395,-1.6045760753$ $\mathrm{H},-0.0128169306,-0.8900597959,-1.6102308987$ $\mathrm{H},-0.0128156192,0.8900056014,-1.6102608641$ C, $-0.9337083909,0.0000160828,0.9145219865$ $\mathrm{H}, 1.0395337661,-0.8830834559,0.703777692$ $\mathrm{H}, 1.0395350673,0.8831056184,0.7037479602$ $\mathrm{H},-0.9209136394,0.0000346487,2.0179720519$ $\mathrm{H},-1.4998069337,0.8900436716,0.586187415$ $\mathrm{H},-1.499808245,-0.8900217257,0.5862173804$

$$
\operatorname{Pr}^{\bullet}: H=-118.374044
$$

$C, 1.321934115,-0.2033983354,-0.0997635028$ C, $0.0454385444,0.5596138496,0.0413862726$ $\mathrm{H}, 2.279606668,0.2364437702,0.1927686601$ $\mathrm{H}, 1.3447901752,-1.1579421389,-0.6351910906$ C, $-1.2097212038,-0.332741715,0.0689603026$ $\mathrm{H}, 0.0818852611,1.1885849691,0.9532560218$ $\mathrm{H},-0.058048205,1.2903423395,-0.7958824122$ $\mathrm{H},-2.1312402754,0.2693249475,0.1409921039$ $\mathrm{H},-1.2785826136,-0.9439756701,-0.8480836755$ $\mathrm{H},-1.1843197439,-1.0236210124,0.9286419583$

$i-\operatorname{Pr}-\mathrm{H}: \mathrm{H}=-119.032998$

C, $0.5023166048,0.0000226051,-1.2065996133$ C, $0.4873484857,0.000016827,0.3299509718$ $\mathrm{H}, 1.0395287221,-0.8830737173,0.703770128$ $\mathrm{H}, 1.5315974481,0.0000621023,-1.6045250423$ $\mathrm{H},-0.0127347732,-0.8900328235,-1.610250797$ $\mathrm{H},-0.0128007452,0.8900425837,-1.610244803$ C, $-0.9337438558,-0.0000375013,0.9145091576$ $\mathrm{H}, 1.0394636929,0.8831454864,0.7037761717$ $\mathrm{H},-0.9209430271,-0.0000413509,2.0179582205$ $\mathrm{H},-1.4998519762,0.8899807307,0.5861800022$ $\mathrm{H},-1.4997867461,-0.8900946434,0.5861730186$

$i-\operatorname{Pr}^{\bullet}: \mathrm{H}=-118.381408$ 
$C,-0.1870247819,0.0638265685,-1.303151651$ C, $0.5182574506,-0.1346417191,0.0000001469$ $\mathrm{H}, 1.4868596039,-0.6474429821,0.0000003944$ $\mathrm{H}, 0.5164854434,0.1273357086,-2.1508343297$ $\mathrm{H},-0.8912772694,-0.7679090059,-1.5360289541$ $\mathrm{H},-0.801257664,0.9852618578,-1.2979178096$ $\mathrm{C},-0.1870255905,0.0638262992,1.3031515482$ $\mathrm{H}, 0.5164841089,0.127335264,2.1508346765$ $\mathrm{H},-0.8012584693,0.9852615895,1.2979175161$ $\mathrm{H},-0.8912782224,-0.7679093234,1.5360282424$

\section{$t-\mathrm{Bu}-\mathrm{H}: \mathrm{H}=-158.318119$}

C, $0.1843534894,-0.3192789792,-1.4226527562$ C, $0.1773400947,-0.3086446237,0.1176098097$ $\mathrm{H}, 0.7022961567,-1.2217319098,0.4653567849$ $\mathrm{H}, 1.2135796198,-0.3154061958,-1.8225932847$ $\mathrm{H},-0.3302283993,-1.2100046036,-1.8239347627$ $\mathrm{H},-0.3320079372,0.5727110966,-1.8233982872$ C, $-1.2612390386,-0.3551197126,0.6659465059$ C, $0.9407753244,0.9112516608,0.6665190671$ $\mathrm{H},-1.2730866199,-0.370841329,1.7699624173$ $\mathrm{H},-1.8348379304,0.5320069124,0.3389889011$ $\mathrm{H},-1.8009976076,-1.2501563084,0.3099499765$ $\mathrm{H}, 0.4560046856,1.8529542799,0.34870123$ $\mathrm{H}, 0.969087552,0.9072109801,1.7703070315$ $\mathrm{H}, 1.9828112614,0.9340070058,0.3021242341$

\section{$t-\mathrm{Bu}^{\bullet}: \mathrm{H}=-157.671951$}

C, $0.2205416585,-0.38526425,-1.4252621368$ C, $0.0651028448,-0.1139676948,0.0422047202$ $\mathrm{H}, 1.2838296013,-0.4469446788,-1.7210576044$ $\mathrm{H},-0.2753639238,-1.3268468168,-1.7237733193$ $\mathrm{H},-0.2306586119,0.4170073188,-2.0549930707$ $C_{,}-1.2417758147,-0.4330132039,0.7069004224$ C, $1.0019276959,0.8519056994,0.7058790267$ $\mathrm{H},-1.1341252994,-0.5199871827,1.8035486301$ $\mathrm{H},-2.010761797,0.3556233414,0.5320037193$ $\mathrm{H},-1.676077901,-1.3759627767,0.3274246236$ $\mathrm{H}, 0.7129581822,1.9139698046,0.5262216894$ $\mathrm{H}, 1.0195745143,0.7187563818,1.8029939798$ $\mathrm{H}, 2.0358469276,0.7464213044,0.3292991567$

\section{$c-\mathrm{Pr}-\mathrm{H}: \mathrm{H}=-117.810073$}

$C_{,}-0.4376106338,0.0000009191,-0.7578162387$ $C_{,}-0.4375245248,-0.0000007195,0.7579961454$ C, $0.8751176708,-0.0000001996,-0.0001033188$ $\mathrm{H},-0.7357101745,-0.917687536,-1.2742352169$ $\mathrm{H},-0.7357097556,0.9176906267,-1.2742332328$ $\mathrm{H},-0.7356503595,-0.9176922981,1.2743465038$ $\mathrm{H},-0.7356499407,0.9176898788,1.2743484878$ $\mathrm{H}, 1.471412788,0.9176830935,-0.0003420426$ $\mathrm{H}, 1.4714123691,-0.9176837644,-0.0003440266$

\section{$c-\operatorname{Pr}^{\bullet}: \mathrm{H}=-117.142599$}

$C, 0.7861445639,0.0513968011,-0.3308727659$ $C_{,}-0.7570814758,0.0513887702,-0.3928466797$ $C,-0.0357685885,-0.0308677692,0.8906850473$ $\mathrm{H}, 1.3374394521,-0.8351937755,-0.6746970112$ $\mathrm{H}, 1.2903269808,0.9965487109,-0.5763173684$ $\mathrm{H},-1.2790213015,-0.8352073432,-0.7797706634$ $\mathrm{H},-1.2399683902,0.9965355754,-0.6779306044$ $\mathrm{H},-0.0685437387,-0.7541899798,1.7069220377$

\footnotetext{
$c-\mathrm{Bu}-\mathrm{H}: \mathrm{H}=-157.097527$
}

$C,-1.0783104541,0.1625097389,0.0908759917$ C, $0.1099825889,0.0141331523,1.0886347026$ C, $1.0824389211,0.0986009195,-0.1265905164$ C, $-0.1141110542,-0.2752439873,-1.0529201823$ $\mathrm{H},-1.983571876,-0.4423229949,0.2673462558$ $\mathrm{H},-1.3810220845,1.2181791658,-0.0201068634$ $\mathrm{H}, 0.1265391234,-0.9893388091,1.5481554755$ $\mathrm{H}, 0.2128299444,0.7653478653,1.8896101029$ $\mathrm{H}, 1.9677367564,-0.55919552,-0.1303593899$ $\mathrm{H}, 1.418234587,1.1353883095,-0.3018122902$ $\mathrm{H},-0.1637516761,-1.3642283557,-1.2262358946$ $\mathrm{H},-0.1969947844,0.2361713992,-2.0265973695$

\section{$c-\mathrm{Bu}^{\bullet}: \mathrm{H}=-156.444559$}

$C, 1.0464380664,-0.0738937911,0.0290977833$ C, $-0.0316170426,0.0044360926,-1.1088706212$ $C,-1.0803158419,0.0761486765,-0.0300374768$ C, $-0.0931866157,0.00444537,1.1053996701$ $\mathrm{H}, 1.6266157385,-1.0104211021,0.0452376872$ $\mathrm{H}, 1.7524844909,0.7717254023,0.0487240988$ $\mathrm{H},-0.0770555679,-0.8818644979,-1.7756023661$ $\mathrm{H}, 0.0479493707,0.8911699206,-1.7718231608$ $\mathrm{H},-2.1717218644,0.1532574158,-0.0604008815$ $\mathrm{H},-0.1756001731,-0.8818594615,1.7685728824$ $\mathrm{H},-0.050583392,0.8911742346,1.7717556078$

\section{$c-\mathrm{Pe}-\mathrm{H}: \mathrm{H}=-196.409585$}

$C,-1.0496281198,-0.0507396202,-0.7671848698$ $C,-1.0012105179,-0.2844189586,0.7608069064$ C, $0.3072463053,0.4119236727,1.1823988926$ C, $1.2993145655,0.0048718859,0.0680270661$ C, $0.440626305,-0.0809667779,-1.2338229186$ $\mathrm{H},-1.6766725215,-0.7902974233,-1.2934337925$ $\mathrm{H},-1.4890575517,0.9425614813,-0.9724903028$ $\mathrm{H},-0.9321941929,-1.3674491266,0.9791581208$ $\mathrm{H},-1.8934302851,0.0990843477,1.2852426258$ $\mathrm{H}, 0.1608350943,1.5091727688,1.1807563862$ $\mathrm{H}, 0.6515722126,0.1290873716,2.1921549996$ $\mathrm{H}, 2.1477464514,0.7037637542,-0.025683782$ $\mathrm{H}, 1.7281591469,-0.9864723976,0.3030257421$ $\mathrm{H}, 0.6557725042,0.7598034615,-1.9152874746$ $\mathrm{H}, 0.6691779128,-1.0032754485,-1.794792983$

\section{$c-\mathrm{Pe}^{\bullet}: \mathrm{H}=-195.761867$}

$C,-1.1696654393,-0.2343246319,0.5611704045$ C, $-0.8632429999,0.4024959245,-0.821734091$ C, $0.6011413323,-0.0060205758,-1.1050028116$ C, $1.2851439502,0.0596428633,0.2877242642$ C, $0.1690515383,-0.2557204928,1.2427156238$ $\mathrm{H},-1.9452233824,0.3181844098,1.1278515455$ $\mathrm{H},-1.5768646038,-1.2639355979,0.431425158$ $\mathrm{H},-0.9341715,1.5032669619,-0.7434849743$ $\mathrm{H},-1.5627010406,0.0823020529,-1.6124043785$ $\mathrm{H}, 0.6315714968,-1.0455306839,-1.4809594494$ $\mathrm{H}, 1.0903174951,0.632262004,-1.8601367069$ $\mathrm{H}, 2.1498190369,-0.6276716947,0.3761526115$ $\mathrm{H}, 1.6991985109,1.0788838242,0.4678642493$ $\mathrm{H}, 0.3134836977,-0.4741998001,2.304451606$ 
$C,-1.1285094086,-0.5899108566,-0.7717170017$ C, $-1.1281755743,-0.5918376909,0.7699498616$ C, $0.3074766655,-0.5884025977,1.3322805281$ C, $1.1285093165,0.5899107857,0.7717176882$ C, $1.1281750902,0.591839401,-0.7699491662$ C, $-0.307476174,0.5884007111,-1.3322812733$ $\mathrm{H}, 0.2877577006,-0.5527988274,2.4371953842$ $\mathrm{H},-1.6613553074,0.3098454654,1.1331918574$ $\mathrm{H},-1.6902314252,-1.4632548082,1.1537144127$ $\mathrm{H},-0.6914133006,-1.5417654612,-1.1352907733$ $\mathrm{H},-2.1643901692,-0.5547244519,-1.1568165526$ $\mathrm{H}, 0.6914114694,1.5417638368,1.1352938952$ $\mathrm{H}, 2.1643907217,0.5547264832,1.1568154264$ $\mathrm{H}, 1.6902264323,1.4632595418,-1.1537138012$ $\mathrm{H}, 1.6613594532,-0.3098408287,-1.1331911486$ $\mathrm{H},-0.8061139107,1.5400512759,-1.0583064136$ $\mathrm{H},-0.2877542462,0.5527918435,-2.4371961234$ $\mathrm{H}, 0.8061130896,-1.5400525843,1.0583000166$

\section{$\mathrm{Cy}^{\bullet}: \mathrm{H}=-235.049680$}

C, $-1.2257586777,-0.225441813,-0.8676457814$ $C_{,}-1.3476780388,0.0359281992,0.6035865902$ C, $-0.1693231165,-0.2303480279,1.4914379025$ C, $1.1503388774,0.3047098736,0.8790106993$ C, $1.3081542352,-0.1449389561,-0.5861139071$ C, $0.1107441215,0.3095378777,-1.4424664611$ $\mathrm{H},-0.3295171448,0.1925286009,2.5000018649$ $\mathrm{H},-2.3368095645,0.1948554285,1.046866096$ $\mathrm{H},-1.2614512656,-1.32545564,-1.0627420731$ $\mathrm{H},-2.0841899625,0.2006775142,-1.418287302$ $\mathrm{H}, 1.1422078799,1.4110127956,0.9184017006$ $\mathrm{H}, 2.0121234841,-0.0314658368,1.4841990515$ $\mathrm{H}, 2.2509183439,0.2500471594,-1.0074773587$ $\mathrm{H}, 1.3868288361,-1.250552795,-0.6236450154$ $\mathrm{H}, 0.0776594141,1.4159566877,-1.4587985837$ $\mathrm{H}, 0.2326175935,-0.0232015957,-2.4895438498$ $\mathrm{H},-0.0492520213,-1.3310852402,1.6441712151$

\section{$\mathrm{Ph}-\mathrm{CH}_{2}-\mathrm{H}: \mathrm{H}=-271.427583$}

$C,-1.6586426515,-0.0122928543,-0.9576177585$ $C_{,}-1.6518383714,-0.004404534,0.4465080235$ $C_{,}-0.4349268542,0.0095785337,1.1457466927$ C, $0.7971754135,0.0134938361,0.4602494286$ C, $0.7747822955,0.0095785232,-0.9495310669$ C, $-0.4392319284,-0.0044045445,-1.6537879953$ $\mathrm{H},-2.6069689406,-0.0196972279,-1.5051341838$ $\mathrm{H},-2.5969751925,-0.0043897603,1.0003805687$ $\mathrm{H},-0.4384308458,0.0198437588,2.2423326883$ $\mathrm{H}, 1.7227016181,0.0198437401,-1.5008586301$ $\mathrm{H},-0.4321326669,-0.0043897791,-2.7492367652$ C, $2.1088801065,-0.0050858723,1.2175624683$ $\mathrm{H}, 2.0189169114,0.4954945908,2.1960844949$ $\mathrm{H}, 2.4443776239,-1.0409784335,1.4112620508$ $\mathrm{H}, 2.9113234327,0.4954945831,0.6503910223$

\section{$\mathrm{Ph}-\mathrm{CH}_{2}^{\bullet}: \mathrm{H}=-270.789072$}

$C,-1.8469817806,0 ., 0.1034487904$ C, $-1.1380294399,1.2190514977,0.0637477025$ C, $0.2534719693,1.2254562358,-0.0141861293$ C, $1.0000060353,0 .,-0.0560074458$ C, $0.2534719693,-1.2254562358,-0.0141861293$ C, $-1.1380294399,-1.2190514977,0.0637477025$ $\mathrm{H},-2.9398650693,0 ., 0.1646504881$
$\mathrm{H},-1.6842200668,2.1681050635,0.0943433221$ $\mathrm{H}, 0.7994577345,2.1751437955,-0.0447521081$ $\mathrm{H}, 0.7994577345,-2.1751437955,-0.0447521081$ $\mathrm{H},-1.6842200668,-2.1681050635,0.0943433221$ C, $2.409540825,0 .,-0.1349925528$

$\mathrm{H}, 2.9743444513,0.9356818155,-0.1666322723$

$\mathrm{H}, 2.9743444513,-0.9356818155,-0.1666322723$

\section{$\mathrm{H}_{2} \mathrm{C}=\mathrm{CH}-\mathrm{CH}_{2}-\mathrm{H}: \mathrm{H}=-117.820129$}

C, $-0.5196109933,-0.028321787,-1.2007684091$ C, $-0.4554311079,-0.0008680018,0.1402899438$ $\mathrm{H}, 0.3869007865,-0.0333671774,-1.8174869541$ $\mathrm{H},-1.4761824989,-0.046737053,-1.732159757$ $\mathrm{H},-1.3925267321,0.0030877232,0.7153884306$ C, $0.8143808181,0.025612076,0.9470471748$ $\mathrm{H}, 0.8624692158,0.9241769875,1.5899032764$ $\mathrm{H}, 0.8767649241,-0.8454925891,1.6254867847$ $\mathrm{H}, 1.7065420031,0.0197983855,0.2994559628$

\section{$\mathrm{H}_{2} \mathrm{C}=\mathrm{CH}-\mathrm{CH}^{\bullet}: \mathrm{H}=-117.185540$}

C, $0.2031560187,-0.1722969084,-1.2237206598$ C, $-0.4438219619,0.0294099111,-0.0062191945$ $\mathrm{H}, 1.2940042091,-0.2534027624,-1.2769857399$ $\mathrm{H},-0.3524705453,-0.2559452037,-2.1611437931$ $\mathrm{H},-1.5405029607,0.1020689632,-0.0216399731$ C, $0.1898182751,0.1462585803,1.2292220761$ $\mathrm{H},-0.3758612231,0.3041652854,2.1509387174$ $\mathrm{H}, 1.2799165289,0.0828842194,1.3131374582$

\section{$\mathrm{H}_{2} \mathrm{C}=\mathrm{C}(\mathrm{Me})-\mathrm{CH}_{2}-\mathrm{H}: \mathrm{H}=-157.110151$}

C, $0.0047308663,-0.1919419639,-1.4559583119$ C, $0.000390239,-0.0158709683,-0.1204277883$ $\mathrm{H}, 0.9369982755,-0.2005035188,-2.0311734756$ $\mathrm{H},-0.9238349189,-0.3335371333,-2.0196825247$ C, $1.2759381514,0.1804908669,0.6680754076$ $\mathrm{H}, 1.2647928417,1.1462110718,1.2083595063$ $\mathrm{H}, 1.3914114093,-0.6044944647,1.4395103745$ $\mathrm{H}, 2.1674971288,0.1595431489,0.0210584226$ C, $-1.2803295433,-0.002262346,0.6838621225$ $\mathrm{H},-1.4000479527,0.9557013867,1.2248049776$ $\mathrm{H},-2.1677258145,-0.1504101593,0.0478360925$ $\mathrm{H},-1.2734692471,-0.7950038941,1.4559780511$

\section{$\mathrm{H}_{2} \mathrm{C}=\mathrm{C}(\mathrm{Me})-\mathrm{CH}^{\bullet}: \mathrm{H}=-156.472777$}

C, $1.4262470284,0.2304399961,0.0323267751$ C, $0.0494864154,-0.0082043413,0.0772048779$ $\mathrm{H}, 1.9469602435,0.4084397092,-0.9129783446$ $\mathrm{H}, 2.0261737132,0.2467605308,0.9474236071$ C, $-0.7534376475,0.0169021739,-1.216908384$ $\mathrm{H},-1.6311034427,-0.6476557846,-1.160955526$ $\mathrm{H},-1.1241556172,1.0359328387,-1.4284667066$ $\mathrm{H},-0.1412987799,-0.2961829172,-2.0784891909$ C, $-0.6098818412,-0.2484855975,1.2863192893$ $\mathrm{H},-1.6850832661,-0.4458660807,1.3238932953$ $\mathrm{H},-0.0659765818,-0.2453416831,2.235917516$

\section{$\mathrm{Me}_{3} \mathrm{SiCH}_{2}-\mathrm{H}: \mathrm{H}=-163.439441$}

Si, $0 ., 0 ., 0$.

C, $1.5569986973,0 ., 1.1004551065$ C, $-1.5569986973,0 ., 1.1004551065$ C, $0 .,-1.5569986973,-1.1004551065$ C, $0 ., 1.5569986973,-1.1004551065$ $\mathrm{H}, 2.4767476922,0 ., 0.4908209846$ $\mathrm{H}, 1.5872612806,0.8906052586,1.7512151122$ $\mathrm{H}, 1.5872612806,-0.8906052586,1.7512151122$ 
$\mathrm{H},-2.4767476922,0 ., 0.4908209846$

$\mathrm{H},-1.5872612806,-0.8906052586,1.7512151122$

$\mathrm{H},-1.5872612806,0.8906052586,1.7512151122$

$\mathrm{H}, 0 .,-2.4767476922,-0.4908209846$

$\mathrm{H},-0.8906052586,-1.5872612806,-1.7512151122$

$\mathrm{H}, 0.8906052586,-1.5872612806,-1.7512151122$

$\mathrm{H}, 0 ., 2.4767476922,-0.4908209846$

$\mathrm{H}, 0.8906052586,1.5872612806,-1.7512151122$

$\mathrm{H},-0.8906052586,1.5872612806,-1.7512151122$

\section{$\mathrm{Me}_{3} \mathrm{SiCH}_{2}^{\bullet}: \mathrm{H}=-162.780742$}

\section{Si, $0 ., 0.0396822654,-0.0253846094$}

C, $1.5640184441,0.0891215417,1.0642145611$

C, $-1.5640184441,0.0891215417,1.0642145611$

C, $0 .,-1.5835112199,-1.0384564854$

C, $0 ., 1.5162068631,-1.1784684011$

$\mathrm{H}, 2.4792987993,0.0912224684,0.447836896$

$\mathrm{H}, 1.5806435256,0.9929137883,1.6961774011$

$\mathrm{H}, 1.6101995344,-0.7901117211,1.7297502312$

$\mathrm{H},-2.4792987993,0.0912224684,0.447836896$

$\mathrm{H},-1.6101995344,-0.7901117211,1.7297502312$

$\mathrm{H},-1.5806435256,0.9929137883,1.6961774011$

$\mathrm{H}, 0 .,-2.4602057256,-0.3669468157$

$\mathrm{H},-0.8914924897,-1.6498258752,-1.6843769941$ $\mathrm{H}, 0.8914924897,-1.6498258752,-1.6843769941$ $\mathrm{H}, 0.9197561924,1.9753121645,-1.5627345675$ $\mathrm{H},-0.9197561924,1.9753121645,-1.5627345675$

\section{$(\mathrm{Me})(\mathrm{H}) \mathrm{C}=\mathrm{CH}-\mathrm{H}: \mathrm{H}=-117.820129$}

$C,-0.5196109933,-0.028321787,-1.2007684091$ $C_{,},-0.4554311079,-0.0008680018,0.1402899438$ $\mathrm{H}, 0.3869007865,-0.0333671774,-1.8174869541$ $\mathrm{H},-1.4761824989,-0.046737053,-1.732159757$ $\mathrm{H},-1.3925267321,0.0030877232,0.7153884306$ C, $0.8143808181,0.025612076,0.9470471748$ $\mathrm{H}, 0.8624692158,0.9241769875,1.5899032764$ $\mathrm{H}, 0.8767649241,-0.8454925891,1.6254867847$ $\mathrm{H}, 1.7065420031,0.0197983855,0.2994559628$

\section{$(\mathrm{Me})(\mathrm{H}) \mathrm{C}=\mathrm{CH}^{\bullet}: \mathrm{H}=-117.145800$}

C, $0.2375025782,-0.0000049793,-1.3642728721$ $\mathrm{C},-0.457080876,0.0000159084,-0.2393006301$ $\mathrm{H}, 1.2876433188,-0.0000378475,-1.6636297418$ $\mathrm{H},-1.5582768292,0.0000525284,-0.2937824399$ $\mathrm{C}, 0.1291792462,-0.0000068966,1.1634163285$ $\mathrm{H},-0.2086266183,0.8888576338,1.7271723782$ $\mathrm{H},-0.2086612705,-0.8888678802,1.7271575001$ $\mathrm{H}, 1.2303157088,-0.0000286293,1.1440253456$

\section{$(t-\mathrm{Bu})(\mathrm{H}) \mathrm{C}=\mathrm{CH}-\mathrm{H}: \mathrm{H}=-235.671734$}

C, $0.3797194424,0.1890484996,2.177309261$ $\mathrm{H},-0.1062708608,1.1667436231,2.2609258692$ C, $0.4671603267,-0.4752001482,1.013108306$ $\mathrm{H}, 0.9708535829,-1.4547450507,1.0060876598$ C, $-0.055704018,-0.0400739367,-0.3484272432$ C, $-1.0586402187,-1.1132984379,-0.8479509265$ $\mathrm{H},-0.5879911023,-2.1114751686,-0.8912091195$ $\mathrm{H},-1.9331881312,-1.1805635428,-0.1785803123$ $\mathrm{H},-1.4190236389,-0.8667556788,-1.8628317549$ C, $-0.7533913503,1.3330910142,-0.298696674$ $\mathrm{H},-1.1184250999,1.6151702574,-1.3015112636$ $\mathrm{H},-1.620951193,1.3191337154,0.3834968648$ $\mathrm{H},-0.0624402123,2.1233448318,0.042373101$
C, $1.1437480255,0.0231931306,-1.3305363308$ $\mathrm{H}, 1.8756059537,0.7857070925,-1.0137233059$ $\mathrm{H}, 1.6681319729,-0.9472785358,-1.3847896975$ $\mathrm{H}, 0.7996696036,0.277341257,-2.3491940611$ $\mathrm{H}, 0.7966758789,-0.2271835302,3.100117665$

\section{$(t-\mathrm{Bu})(\mathrm{H}) \mathrm{C}=\mathrm{CH}^{\bullet}: \mathrm{H}=-234.999065$}

C, $0.3809698944,0.2081072935,2.2250071984$ $\mathrm{H},-0.0253614147,1.1511482711,2.591611931$ C, $0.4788222948,-0.4625735339,1.0922172773$ $\mathrm{H}, 0.985673684,-1.4439182783,1.0976279012$ C, $-0.0417299059,-0.046282491,-0.29911286$ $C,-1.0373945017,-1.1304819259,-0.7794607372$ $\mathrm{H},-0.5624855934,-2.1269925819,-0.8067927091$ $\mathrm{H},-1.9125752577,-1.1899229898,-0.1105728268$ $\mathrm{H},-1.3970384365,-0.900058341,-1.7985194753$ C, $-0.7404841211,1.3256350564,-0.2559858361$ $\mathrm{H},-1.1048360233,1.6034286955,-1.260325065$ $\mathrm{H},-1.6067484872,1.3111603978,0.4275045755$ $\mathrm{H},-0.0490510786,2.1153171217,0.0847749371$ C, $1.1681247674,0.0080708385,-1.2639113774$ $\mathrm{H}, 1.8944780927,0.7753494991,-0.946765631$ $\mathrm{H}, 1.6940000538,-0.9621282817,-1.3024832395$ $\mathrm{H}, 0.8340938934,0.2517650623,-2.2885823887$

\section{Mesityl-H : H = -350.004717}

C, $0.3616488877,1.0805872843,2.7031708437$ $\mathrm{H}, 1.2098026662,1.7865358289,2.7526354767$ $\mathrm{H},-0.5389976787,1.6092159205,3.0562269223$ C, $0.1730651555,0.5372252023,1.3012089782$ C, $1.2482802872,-0.0761224929,0.6228303833$ C, $1.0929033614,-0.6115304434,-0.6659408608$ $C,-0.1737579383,-0.5242073611,-1.2831213047$ $C,-1.2640808883,0.0818127919,-0.6383908185$ C, $-1.0732416182,0.6060412969,0.6582979123$ $\mathrm{H}, 2.2295707205,-0.1324400369,1.1114510925$ $\mathrm{H},-0.3097740435,-0.9378837278,-2.2907213663$ $\mathrm{H},-1.9194015668,1.0742716954,1.1775374922$ C, $-2.6152974461,0.1841942757,-1.3160588201$ $\mathrm{H},-2.8535484692,1.2301153938,-1.5834649081$ $\mathrm{H},-2.648033894,-0.411082846,-2.2431961484$ $\mathrm{H},-3.4265345189,-0.1707728192,-0.6563923258$ C, $2.2516556342,-1.2726556779,-1.3839374315$ $\mathrm{H}, 2.4583255149,-0.7839627199,-2.3529596977$ $\mathrm{H}, 3.1748082765,-1.2312652542,-0.7831943136$ $\mathrm{H}, 2.0392885049,-2.3353812776,-1.6000531287$ $\mathrm{H}, 0.5774418773,0.2705805883,3.4237776134$

\section{Mesityl $^{\bullet}: \mathrm{H}=-349.366545$}

C, $0.3470478543,1.1268879529,2.6572308567$ $\mathrm{H}, 1.3182324535,1.1041157831,3.1591309778$ $\mathrm{H},-0.4890213452,1.588364481,3.189817784$ C, $0.1786469315,0.5800928148,1.3678881628$ $C, 1.2732647078,-0.025774025,0.6669375057$ C, $1.1163356993,-0.5698742593,-0.610035042$ C, $-0.1594598773,-0.5177765191,-1.2209618726$ $C,-1.2703907325,0.0696487197,-0.5695294382$ C, $-1.093815296,0.6084764223,0.7071149636$ $\mathrm{H}, 2.2552404664,-0.0580200408,1.1543008192$ $\mathrm{H},-0.2904526254,-0.9431122314,-2.2239572051$ $\mathrm{H},-1.9444356597,1.067261817,1.2255747023$ C, $-2.6209972482,0.1069491532,-1.2552971866$ $\mathrm{H},-2.5716124216,0.6596915579,-2.2105053026$ 
$\mathrm{H},-2.9800897359,-0.9107289445,-1.4914423639$ $\mathrm{H},-3.381145263,0.5939798684,-0.623470046$ C, $2.2822426199,-1.2069283663,-1.3384776298$ $\mathrm{H}, 2.4852854298,-0.6957727342,-2.2965353959$ $\mathrm{H}, 3.2037190693,-1.1700589056,-0.7353594433$ $\mathrm{H}, 2.0770316801,-2.2659320098,-1.576776444$

\section{$\mathrm{Pe}-\mathrm{H}: \mathrm{H}=-197.600148$}

C, $-0.2618987377,-0.0000015977,-0.1769082138$ $C,-0.2856229422,0.0000154944,1.3616355207$ $\mathrm{H},-0.8163743934,-0.884178792,-0.5513660777$ $\mathrm{H},-0.8163686193,0.8841709543,-0.5513854948$ $\mathrm{C},-1.7062452671,0.0000263114,1.9475105208$ $\mathrm{H}, 0.2688255206,-0.8836563174,1.7338765892$ $\mathrm{H}, 0.2688308928,0.8836921397,1.7338571134$ $\mathrm{H},-1.6915174741,0.000038609,3.0508876108$ $\mathrm{H},-2.2728746416,0.8901001251,1.6206486051$ $\mathrm{H},-2.2728799558,-0.8900514062,1.6206684816$ C, $1.1561178047,-0.0000127757,-0.7744386044$ $\mathrm{H}, 1.7088408311,0.8837891051,-0.3995342289$ $\mathrm{H}, 1.7088340026,-0.8838118028,-0.3995174152$ C, $1.1670259379,-0.0000273063,-2.3110561003$
$\mathrm{H}, 0.6514087261,-0.8903494856,-2.7136217002$ $\mathrm{H}, 0.6514152002,0.8902909649,-2.7136386323$ $\mathrm{H}, 2.1955991372,-0.0000348505,-2.7113335884$

\section{$\mathrm{Pe}^{\bullet}: \mathrm{H}=-196.941340$}

$C, 2.6152249801,-0.2122522934,-0.1795616509$ C, $1.3396938555,0.5510759943,-0.0389968172$ $\mathrm{H}, 3.5674305898,0.2056011355,0.1587354298$ $\mathrm{H}, 2.6460776114,-1.1437991901,-0.7537535143$ C, $0.0779726331,-0.3376463893,-0.0041262757$ $\mathrm{H}, 1.374873252,1.1853241211,0.8698447545$ $\mathrm{H}, 1.2283582258,1.276415281,-0.8814807598$ $\mathrm{H}, 0.0547756081,-0.9668829083,-0.9161221421$ $\mathrm{H}, 0.1525851395,-1.0394899689,0.8488555202$ C, $-1.2318004692,0.4622432492,0.0975754192$ $\mathrm{H},-1.2958589044,1.165109869,-0.7561756534$ $\mathrm{H},-1.2037911513,1.0928292615,1.0076120455$ C, $-2.4838411985,-0.4277624518,0.1277786794$ $\mathrm{H},-3.4070553328,0.1722603121,0.1987381986$ $\mathrm{H},-2.5563102565,-1.0462872939,-0.7844253708$ $\mathrm{H},-2.4645835873,-1.1150292737,0.9921553629$ 


\section{Cartesian coordinates for the Jones series $\mathrm{Rh}(\mathrm{H})(\mathrm{R})(\mathrm{Tp})\left(\mathrm{CNCH}_{3}\right)$ and enthalpies (au) at B3PW91}

\section{Fragment $\mathrm{Rh}(\mathrm{Tp})(\mathrm{CNMe})(\mathrm{H})^{\bullet}: \mathrm{H}=-945.827025$}

$\mathrm{Rh}, 0,-0.5724719635,-0.6675296994,-0.7500498973$ $\mathrm{H}, \mathrm{O},-0.5342264035,-2.1933931873,-1.0745781026$ $\mathrm{N}, 0,-0.9979363811,-1.101003194,1.2691654416$ $\mathrm{N}, 0,-0.4152952873,-0.3601942905,2.2367135337$ $\mathrm{N}, 0,1.5409730265,-0.504577469,-0.0652225146$ $\mathrm{N}, 0,1.7542453704,0.1676787716,1.0844183182$ $\mathrm{N}, 0,-0.7306380074,1.4414739977,-0.1640408801$ $\mathrm{N}, 0,-0.1583408339,1.8105999272,1.000450928$ $\mathrm{N}, 0,-0.1686904107,-0.3172860569,-3.7624544251$ C, $0,-1.7635453147,-2.0124609747,1.8751096444$ C, $0,-1.6847023987,-1.8612693916,3.2649693989$ $\mathrm{H}, \mathrm{O},-2.1859787481,-2.4415947943,4.0246819332$ C, $0,-0.8127380754,-0.7997892365,3.4473455997$ $C, 0,2.726826637,-0.9327605241,-0.4961618592$ C, $0,3.739808998,-0.5317742299,0.3881415333$ $\mathrm{H}, \mathrm{O}, 4.7999134732,-0.7242424624,0.3167829675$ C, $0,3.0718502526,0.1647813581,1.3811021525$ C, $0,-1.2866595135,2.5352864998,-0.6887824969$ C, $0,-1.0750392867,3.6402755429,0.1476911622$ $\mathrm{H}, \mathrm{O},-1.4020030692,4.6586368676,0.0005379678$ C, $0,-0.3514629773,3.1291367431,1.2123823062$ $C, 0,-0.3387280314,-0.4423669025,-2.6040701655$ C, $0,0.0289097059,-0.2179727855,-5.1536238835$ $\mathrm{H}, \mathrm{O}, 0.7997934804,0.5267922489,-5.3746144277$ $\mathrm{H}, 0,-0.9001856813,0.0828666156,-5.6481957413$ $\mathrm{B}, 0,0.574826293,0.7724934846,1.8770119706$ $\mathrm{H}, 0,0.9801438629,1.2958982113,2.8819999128$ $\mathrm{H}, 0,3.4343406702,0.6541830701,2.2735600151$ $\mathrm{H}, 0,2.791615648,-1.5086605312,-1.4087901559$ $\mathrm{H}, \mathrm{O},-0.4456731651,-0.3282525179,4.3472783833$ $\mathrm{H}, \mathrm{0},-2.3211330335,-2.7238321937,1.2831390156$ $\mathrm{H}, 0,0.0378659731,3.6044421012,2.1010588659$ $\mathrm{H}, \mathrm{O},-1.8101653538,2.4736092872,-1.632728224$ $\mathrm{H}, 0,0.3454569278,-1.1834420597,-5.5607800472$

$$
\mathrm{R}=\mathrm{Ph}, \mathrm{H}=-1177.313230
$$

$\mathrm{Rh}, 0.0502967233,-0.4871449057,-0.4765629278$ $\mathrm{H},-0.2717187155,-1.9358052457,-0.8834080833$ $\mathrm{N},-0.7864375241,-0.8196459795,1.4152880145$ $\mathrm{N},-0.1402094702,-0.3668486251,2.5123004505$ $\mathrm{N}, 1.9322608272,-1.0535362318,0.4614330691$ $\mathrm{N}, 2.2127663995,-0.5577276144,1.6834750468$ $\mathrm{N}, 0.5106166129,1.5406306288,0.2735743588$ $\mathrm{N}, 1.0051183768,1.6419014713,1.5248287493$ $\mathrm{N}, 1.213311634,-0.1495795527,-3.2764469241$ C, $-1.8956091992,-1.4354061014,1.8286193609$ C, $-1.9774043647,-1.3858479809,3.2257122993$ $\mathrm{H},-2.7526890219,-1.7913430296,3.8578635233$ C, $-0.8400070885,-0.6977827367,3.6161760064$ C, $2.9433825929,-1.8549248652,0.1215472827$ C, $3.9056231688,-1.8816966421,1.1403907793$ $\mathrm{H}, 4.8326822326,-2.4344379479,1.1645494993$ C, $3.3950895633,-1.0417248687,2.1164693663$ C, $0.4489602678,2.7763962781,-0.2244887081$ C, $0.9126945202,3.7048398043,0.7185632807$ $\mathrm{H}, 0.9831990723,4.7773592989,0.6166236905$ C, $1.2554110073,2.9351893262,1.8180364159$ C, $0.7674774338,-0.2736241212,-2.1986669539$ C, $1.6951284157,0.0192406991,-4.5909500788$
$\mathrm{H}, 2.6379234169,0.5737096824,-4.5770019512$ $\mathrm{H}, 0.9651245975,0.5743517986,-5.1872795451$ $\mathrm{B}, 1.2075197429,0.3793456923,2.3881203307$ $\mathrm{H}, 1.6158879688,0.6818461107,3.4784303335$ $\mathrm{H}, 3.7833640551,-0.756096485,3.0833282645$ $\mathrm{H}, 2.9246961247,-2.373558782,-0.8266492256$ $\mathrm{H},-0.4780998127,-0.4192128877,4.5951615531$ $\mathrm{H},-2.5707548352,-1.865052677,1.1032250558$ $\mathrm{H}, 1.655142423,3.2116060275,2.7829839511$ $\mathrm{H}, 0.0617318618,2.9353473141,-1.221241802$ $\mathrm{H}, 1.8649853421,-0.9556726213,-5.0575292109$ C, $-1.7803430711,-0.0315641482,-1.2318177947$ $C,-2.34861848,-0.6857946991,-2.3400681587$ $C,-3.6222309751,-0.3604753181,-2.8115894004$ C, $-4.3724683749,0.6339572915,-2.1868616287$ $C,-3.8327064901,1.2932768807,-1.0839551929$ C, $-2.5585888969,0.9647018104,-0.6160277193$ $\mathrm{H},-1.7929056065,-1.4746168725,-2.8414584562$ $\mathrm{H},-4.0293528351,-0.8920090942,-3.6695030275$ $\mathrm{H},-5.3643694811,0.8887460021,-2.5514428894$ $\mathrm{H},-4.4053193123,2.0688004676,-0.5791339058$ $\mathrm{H},-2.1672069056,1.4919089101,0.2495080329$

$$
\mathrm{R}=t \text {-Bu-vinyl, } \mathrm{H}=-1180.857918
$$

$\mathrm{Rh}, 0.2725193811,-0.3961620864,-0.3144035461$ $\mathrm{H},-0.0847152781,-1.8322413454,-0.7383009741$ $\mathrm{N},-0.5731294881,-0.7334803193,1.5788921824$ $\mathrm{N}, 0.0783869103,-0.3057832482,2.6815289311$ $\mathrm{N}, 2.1527830488,-0.984617347,0.6196525427$ $\mathrm{N}, 2.4325435955,-0.4938697457,1.8444867857$ $\mathrm{N}, 0.730002514,1.6206367068,0.4601091751$ $\mathrm{N}, 1.2203284711,1.7091408166,1.7134408217$ $\mathrm{N}, 1.3619989433,-0.0969559918,-3.1443074823$ $C,-1.6957003667,-1.3302975069,1.9816704272$ $C,-1.7816680753,-1.2926720249,3.3793531458$ $\mathrm{H},-2.566356765,-1.6894331169,4.0055426725$ $C,-0.6316536286,-0.6320414475,3.7804393518$ C, $3.1705223759,-1.7738497965,0.2721364081$ C, $4.1363247049,-1.7984262137,1.2879585804$ $\mathrm{H}, 5.0685375507,-2.3426980024,1.3060281093$ C, $3.6211013433,-0.9694210391,2.2706531011$ C, $0.6422128986,2.86364398,-0.0147755324$ C, $1.0841595669,3.7846798601,0.946095356$ $\mathrm{H}, 1.13367911,4.8600532661,0.8636141799$ C, $1.4416334665,3.001949179,2.0314656798$ $C,-1.5352408676,0.1553516431,-1.0102534867$ $\mathrm{H},-1.8521752713,1.1186155675,-0.5974728993$ C, $-2.3719378346,-0.489537041,-1.8342358583$ $\mathrm{H},-2.0678916061,-1.4537106231,-2.250745842$ C, $0.9500429618,-0.2009102225,-2.0505319015$ C, $1.8006559069,0.0173554894,-4.4790841106$ $\mathrm{H}, 2.7948922667,0.4725170103,-4.5114184062$ $\mathrm{H}, 1.1065239992,0.6416955365,-5.0493310834$ $\mathrm{B}, 1.4285320446,0.4353430355,2.5611466353$ $\mathrm{H}, 1.8410560456,0.7269847975,3.6530327132$ $\mathrm{H}, 4.0095246933,-0.686588458,3.2382771835$ $\mathrm{H}, 3.1540166512,-2.2856147307,-0.6798494428$ $\mathrm{H},-0.2677924415,-0.3699027448,4.7632540154$ $\mathrm{H},-2.3732795168,-1.7388404556,1.2464294325$ $\mathrm{H}, 1.833123829,3.2690239354,3.0023960858$ $\mathrm{H}, 0.264536881,3.0329723869,-1.0137315998$ 
$\mathrm{H}, 1.8491994826,-0.9714835879,-4.9451402434$ C, $-3.7577202649,-0.0520234292,-2.2874238819$ C, $-4.7745498191,-1.1234880374,-1.8491868279$ $\mathrm{H},-4.5000285761,-2.1110825179,-2.237507048$ $\mathrm{H},-4.8205178013,-1.1949153085,-0.7571930406$ $\mathrm{H},-5.7804693691,-0.8864840245,-2.2172728608$ C, $-4.1750208723,1.3035095697,-1.7096807039$ $\mathrm{H},-5.1783630341,1.5736817223,-2.0585675175$ $\mathrm{H},-4.1943930807,1.2834270641,-0.6150067225$ $\mathrm{H},-3.4875042943,2.0980189398,-2.0194034899$ $C,-3.7607383425,0.0318458679,-3.8258442187$ $\mathrm{H},-3.0632361193,0.8009588747,-4.1750911424$ $\mathrm{H},-3.4597169266,-0.9228039241,-4.2729207479$ $\mathrm{H},-4.7596196853,0.2789193674,-4.2063487957$

\section{$\mathrm{R}=\mathrm{Me}, \mathrm{H}=-985.704654$}

$\mathrm{Rh},-0.5308361586,-0.4180135503,-0.7951784708$ $\mathrm{H},-0.8842015536,-1.8603879157,-1.2126509304$ $\mathrm{N},-1.3634202669,-0.7556133348,1.1058260475$ $\mathrm{N},-0.722079377,-0.2899763432,2.1991229717$ $\mathrm{N}, 1.3527808959,-0.9822973906,0.1571882584$ $\mathrm{N}, 1.6315971702,-0.457713702,1.3678747541$ $\mathrm{N},-0.0980992402,1.6147165971,-0.051073473$ $\mathrm{N}, 0.3908267381,1.7291066706,1.2005155266$ $\mathrm{N}, 0.5680776549,-0.1341723801,-3.6187421884$ C, $-2.4494411907,-1.4056661105,1.5288865462$ C, $-2.5229436059,-1.361172515,2.9267822269$ $\mathrm{H},-3.280157383,-1.7914520343,3.5645078488$ C, $-1.4018601197,-0.6425585528,3.3088007106$ C, $2.3758148955,-1.7724181912,-0.171858856$ C, $3.344230991,-1.7633254511,0.8422305425$ $\mathrm{H}, 4.280542727,-2.3000531283,0.8724410503$ C, $2.8245624978,-0.9132799652,1.8042040372$ C, $-0.1996424145,2.8490007709,-0.5447026922$ C, $0.2313764292,3.7903517371,0.4014908269$ $\mathrm{H}, 0.2683405174,4.8647883825,0.3018794594$ C, $0.5972612224,3.029208691,1.4990225314$ C, $-2.375338154,0.1127631125,-1.5559815823$ $\mathrm{H},-2.3551657543,1.156529221,-1.8930935107$ C, $0.1524963382,-0.2341286506,-2.5242259077$ C, $1.0633171198,-0.0424360117,-4.9351139854$ $\mathrm{H}, 2.0691230475,0.3883418637,-4.9306886972$ $\mathrm{H}, 0.4105835441,0.5940022138,-5.5401574246$ $B, 0.6158838326,0.4729727825,2.0662264506$ $\mathrm{H}, 1.0217440113,0.7869437907,3.1545474579$ $\mathrm{H}, 3.2125351277,-0.6026510675,2.7634726926$ $\mathrm{H}, 2.360552645,-2.3096233765,-1.1097557181$ $\mathrm{H},-1.0384991706,-0.3570908636,4.2852186511$ $\mathrm{H},-3.1123282131,-1.8684094662,0.8122611777$ $\mathrm{H}, 0.9853375067,3.3156455763,2.4657973927$ $\mathrm{H},-0.5794566553,3.0006037543,-1.5455181658$ $\mathrm{H}, 1.1067189403,-1.0359097952,-5.3921112542$ $\mathrm{H},-3.1154889567,0.0317912038,-0.7533293727$ $\mathrm{H},-2.6987614849,-0.5137065076,-2.3931113879$

\section{$\mathrm{R}=\mathrm{Pe}, \mathrm{H}=-1142.790840$}

$\mathrm{Rh}, 0.2522148442,-0.2374768682,-0.3233640827$ $\mathrm{H}, 0.3476950643,-1.7392437897,-0.6601508919$ $\mathrm{N},-0.2418168543,-0.7071344659,1.6667893514$ $\mathrm{N}, 0.3314644717,-0.0104066937,2.6713288486$ $\mathrm{N}, 2.3108179676,-0.1357525286,0.426862177$ $\mathrm{N}, 2.5268841146,0.5140289615,1.588665981$ $\mathrm{N}, 0.0951160431,1.864785217,0.325176164$ $\mathrm{N}, 0.6493509345,2.1935529552,1.5100812504$
$\mathrm{N}, 0.9482564705,0.212597208,-3.2524653559$ C, $-1.0374196256,-1.623372791,2.2220673079$ C, $-0.9858605521,-1.5233841378,3.6180491235$ $\mathrm{H},-1.5135819274,-2.121478615,4.3453688156$ C, $-0.1010083484,-0.4844125093,3.8570817398$ C, $3.4935156305,-0.5847005121,0.004383325$ C, $4.5038152578,-0.2204079139,0.9061376083$ $\mathrm{H}, 5.5598049735,-0.4368147159,0.8448145924$ C, $3.8392140012,0.4764578693,1.9014573883$ C, $-0.4352865499,2.9789189301,-0.1801632319$ C, $-0.224138898,4.0584628406,0.6898847916$ $\mathrm{H},-0.533779815,5.0850431649,0.5636406925$ C, $0.4704972098,3.5088437148,1.754377618$ $C,-1.7507612456,-0.3140463407,-0.8574122064$ $\mathrm{H},-1.982778037,0.5946951784,-1.4325434466$ C, $0.6785132949,0.0482230527,-2.1203480522$ C, $1.2827368698,0.3760129571,-4.6119230521$ $\mathrm{H}, 1.9657687506,1.2221127882,-4.7334513278$ $\mathrm{H}, 0.380817099,0.5639773389,-5.2022120189$ $\mathrm{B}, 1.3434469159,1.1197050887,2.3734642358$ $\mathrm{H}, 1.7347466043,1.6052137735,3.4025692705$ $\mathrm{H}, 4.2011128204,0.9433846323,2.8060474783$ $\mathrm{H}, 3.5576208223,-1.1495646689,-0.9150900138$ $\mathrm{H}, 0.251192419,-0.048242253,4.7804515477$ $\mathrm{H},-1.5986838343,-2.2995651801,1.5935588231$ $\mathrm{H}, 0.8471795864,3.9541047404,2.6638115502$ $\mathrm{H},-0.9438355501,2.9499811251,-1.133789876$ $\mathrm{H}, 1.7692074286,-0.5275427173,-4.9923525764$ $\mathrm{H},-2.2954303758,-0.2244226476,0.0920276447$ C, $-2.2513915894,-1.5350945603,-1.6255838712$ $\mathrm{H},-1.7027552496,-1.635538549,-2.5729511132$ $\mathrm{H},-2.026856286,-2.4501234009,-1.0597340704$ $\mathrm{C},-3.754880028,-1.4911211865,-1.9226233214$ $\mathrm{H},-3.9849237568,-0.5858926254,-2.5041087463$ $\mathrm{H},-4.3074138464,-1.3874275491,-0.9772101946$ C, $-4.2707645328,-2.7190359974,-2.6751422632$ $\mathrm{H},-3.7226700069,-2.8189405084,-3.6224323821$ $\mathrm{H},-4.0344388483,-3.6220435631,-2.0953436666$ C, $-5.7710340184,-2.6732057918,-2.956500638$ $\mathrm{H},-6.0345448843,-1.7990812042,-3.5632397127$ $\mathrm{H},-6.1089881774,-3.5648639869,-3.4954445488$ $\mathrm{H},-6.3463608295,-2.6103134611,-2.0256957967$

\section{$\mathrm{R}=c-\mathrm{Pe}, \mathrm{H}=-1141.596603$}

$\mathrm{Rh},-0.3658273353,0.1692526279,-0.4780643087$ $\mathrm{H},-0.7742041865,0.130348329,-1.960967369$ $\mathrm{N}, 0.8500227508,-1.5250383398,-0.7726939519$ $\mathrm{N}, 2.0810923122,-1.5625112827,-0.21964378$ $\mathrm{N}, 1.4792695403,1.2958485194,-0.8927342659$ $\mathrm{N}, 2.6268081027,0.8760234377,-0.3236549112$ $\mathrm{N}, 0.385444302,0.1585775241,1.6189227184$ $\mathrm{N}, 1.6986682291,-0.0994877845,1.7963991678$ $\mathrm{N},-2.1376886054,2.6374406907,-0.2865552523$ C, $0.6939431365,-2.6530176875,-1.469523771$ C, $1.8431724005,-3.4462830634,-1.3664736966$ $\mathrm{H}, 2.0240288005,-4.4118861576,-1.8137532831$ C, $2.6991677196,-2.7102585038,-0.5635740864$ C, $1.781297555,2.3545305049,-1.6459917988$ C, $3.1529641693,2.634658342,-1.5656450546$ $\mathrm{H}, 3.7007009386,3.4235806862,-2.0589202569$ C, $3.651059702,1.6638215068,-0.7124492312$ $C,-0.1181937784,0.437257536,2.822872357$ C, $0.8793525719,0.3551835811,3.8047632825$ $\mathrm{H}, 0.7814414768,0.5177170923,4.8676316637$ 
C, $2.0213676152,0.0111135713,3.102301868$ $C,-1.4637785874,1.6763253571,-0.3469730506$ C, $-2.9671846366,3.7749749477,-0.2189034296$ $\mathrm{H},-2.4646599567,4.5756874632,0.3319470806$ $\mathrm{H},-3.9031739891,3.529387662,0.2921448962$ $\mathrm{B}, 2.623657692,-0.3667991745,0.5935014886$ $\mathrm{H}, 3.7402585246,-0.6028470189,0.9749162499$ $\mathrm{H}, 4.6537740574,1.4755402769,-0.3568994731$ $\mathrm{H}, 1.0053871967,2.8532421799,-2.2097923177$ $\mathrm{H}, 3.6997459415,-2.9171748667,-0.2130109889$ $\mathrm{H},-0.2270539391,-2.8322353405,-2.0051679865$ $\mathrm{H}, 3.0352192898,-0.1623873236,3.4328180505$ $\mathrm{H},-1.1669052582,0.6748773583,2.9266897051$ $\mathrm{H},-3.2005271032,4.1325529087,-1.2265116507$ $C,-2.0198907534,-1.0593240218,-0.1835057505$ C, $-2.8181524933,-0.9204597205,1.1206326835$ C, $-4.0813737246,-1.7858554014,0.9026675568$ $C,-4.2673041366,-1.8826269013,-0.6364249778$ $C,-3.133933566,-1.0278253233,-1.2393536707$ $\mathrm{H},-1.5837120995,-2.0696166088,-0.1858433591$ $\mathrm{H},-3.1127558691,0.1273699535,1.2759931711$ $\mathrm{H},-2.2518850268,-1.23271058,2.0027321406$ $\mathrm{H},-3.936738774,-2.7827827604,1.3333862268$ $\mathrm{H},-4.959215069,-1.357650693,1.3993854122$ $\mathrm{H},-4.1705575566,-2.9239019406,-0.9645765287$ $\mathrm{H},-5.2588970405,-1.5455622183,-0.9584638036$ $\mathrm{H},-2.8062414728,-1.3876154398,-2.2208959882$ $\mathrm{H},-3.4804828374,0.0044369571,-1.3842485962$

\section{$\mathrm{R}=\mathrm{Cy}, \mathrm{H}=-1180.875405$}

$\mathrm{Rh},-0.1623577552,0.2305926887,-0.4985551799$ $\mathrm{H},-0.5457911992,0.1463107312,-1.9831919421$ $\mathrm{N}, 0.692075551,-1.6924947385,-0.6175877473$ $\mathrm{N}, 1.8900895309,-1.9243802792,-0.0397363029$ $\mathrm{N}, 1.8734255307,0.9273815445,-0.9814396475$ $N, 2.9181776326,0.3312372846,-0.3738042534$ $\mathrm{N}, 0.6001307175,0.2876380891,1.606515702$ $\mathrm{N}, 1.8339725527,-0.2183395953,1.8191290124$ $\mathrm{N},-1.4192013441,3.0038980524,-0.5521327377$ C, $0.3128409681,-2.8269322691,-1.2108277921$ C, $1.2781939221,-3.8214958693,-1.0125778435$ $\mathrm{H}, 1.2608362085,-4.8414660113,-1.3654435206$ C, $2.2643013703,-3.2000373637,-0.2641797266$ C, $2.375946319,1.8248544503,-1.8308410432$ C, $3.7771528217,1.8180825183,-1.7747803265$ $\mathrm{H}, 4.46840694,2.4263284011,-2.3386664627$ C, $4.0772032037,0.8477618206,-0.8329436266$ C, $0.2031607595,0.8266905178,2.76149917$ $C, 1.1874678642,0.664296576,3.7464984393$ $\mathrm{H}, 1.1556556645,0.9831794032,4.7775115947$ C, $2.210084405,-0.005855352,3.0979669383$ $C_{,}-0.9480608303,1.9274057836,-0.5180673787$ $C,-2.0104409359,4.282394269,-0.6007380875$ $\mathrm{H},-1.3564826138,5.0186700907,-0.1239063861$ $\mathrm{H},-2.9716658285,4.2738633429,-0.0777008943$ $\mathrm{B}, 2.6705285704,-0.7904903326,0.6582053441$ $\mathrm{H}, 3.7189678879,-1.2106442886,1.0734950653$ $\mathrm{H}, 5.0247815083,0.488346777,-0.4584043455$ $\mathrm{H}, 1.7110465724,2.4202547139,-2.4408255425$ $\mathrm{H}, 3.2029062727,-3.569277066,0.1225229028$ $\mathrm{H},-0.6245052253,-2.8686625098,-1.7463558992$ $\mathrm{H}, 3.1736829226,-0.3457183787,3.4489466161$ $\mathrm{H},-0.7669758681,1.2968711915,2.8327855075$ $\mathrm{H},-2.1787933031,4.5810822513,-1.6400016187$
$C,-4.0112912264,-0.9871534406,1.4349532817$ $C,-2.6926327207,-0.2382708221,1.1822143154$ $C,-2.0474273438,-0.6286799797,-0.1510762009$ $C,-3.0547140443,-0.4453942243,-1.2950314354$ $C,-4.3724308327,-1.1939290015,-1.0451684434$ $C,-5.0023636566,-0.7897310433,0.2879269805$ $\mathrm{H},-2.8981951839,0.8439497431,1.195939023$ $\mathrm{H},-2.0038282419,-0.4410547669,2.0077758326$ $\mathrm{H},-3.7967881088,-2.0603874348,1.542819651$ $\mathrm{H},-4.453369397,-0.6621986581,2.3863547133$ $\mathrm{H},-3.2767713611,0.6250739744,-1.4220901124$ $\mathrm{H},-2.6189106101,-0.7760376141,-2.2449984374$ $\mathrm{H},-5.072649536,-1.0149000749,-1.8719475632$ $\mathrm{H},-4.1752419924,-2.2762053699,-1.0335720628$ $\mathrm{H},-5.2966495493,0.2697178168,0.2418325358$ $\mathrm{H},-5.9224075697,-1.359629837,0.4705087014$ $\mathrm{H},-1.806433715,-1.7003076766,-0.0850024854$

\section{$\mathrm{R}=$ Methallyl, $\mathrm{H}=-1102.310989$}

$\mathrm{Rh}, 0.0404962854,-0.2425344378,0.5941868858$ $\mathrm{H}, 0.4848299025,-1.6036824759,1.16432963$ $\mathrm{N}, 1.4197397558,-0.4566610206,-0.9778937035$ $\mathrm{N}, 1.0402589339,-0.1300966386,-2.2333140958$ $\mathrm{N},-1.3734891577,-1.1478273125,-0.7954733386$ $\mathrm{N},-1.3897489703,-0.6930628797,-2.0648130172$ $\mathrm{N},-0.4917823462,1.6708992155,-0.3612455302$ $\mathrm{N},-0.6210658419,1.6795306175,-1.7035127696$ $\mathrm{N},-1.9192153622,-0.1459248253,2.9247038001$ $C, 2.6626678086,-0.9356959593,-1.0467108237$ C, $3.1093015044,-0.9169109101,-2.3744546873$ $\mathrm{H}, 4.0682368479,-1.2351788115,-2.7545294595$ C, $2.0436435459,-0.39989231,-3.0927424594$ C, $-2.3031753525,-2.1003654748,-0.7077394267$ C, $-2.9415293703,-2.2719350626,-1.9440870197$ $\mathrm{H},-3.7310143993,-2.9645313406,-2.1943962992$ C, $-2.3240093299,-1.3550521754,-2.7786419222$ C, $-0.7331457488,2.9120117814,0.0628343854$ $C,-1.0272185055,3.7496792264,-1.0227135307$ $\mathrm{H},-1.2633303874,4.8030510446,-1.0051826423$ C, $-0.9426079519,2.9195154944,-2.128112203$ C, $1.4978480189,0.7074905756,1.7649268576$ $\mathrm{H}, 0.9709956729,1.393261139,2.4430257607$ C, $-1.1585730609,-0.1750205162,2.029518669$ $C,-2.8313078824,-0.1476004672,3.9997432594$ $\mathrm{H},-3.8437164482,0.049636964,3.6347829835$ $\mathrm{H},-2.5613755602,0.6262963948,4.7244569218$ $\mathrm{B},-0.3849497896,0.3904568775,-2.5178667481$ $\mathrm{H},-0.5197575574,0.6125941096,-3.6926348138$ $\mathrm{H},-2.4771816588,-1.1287327072,-3.8238964933$ $\mathrm{H},-2.4588677784,-2.6161078797,0.2293596629$ $\mathrm{H}, 1.9250911676,-0.2042598909,-4.1485659767$ $\mathrm{H}, 3.1611431933,-1.2523966206,-0.1412105071$ $\mathrm{H},-1.0846830082,3.1195270974,-3.1802595654$ $\mathrm{H},-0.6776212539,3.1422966926,1.1177826861$ $\mathrm{H},-2.8195696346,-1.1193735478,4.5028043675$ $\mathrm{H}, 2.0539559478,1.3152753857,1.0435650059$ C, $2.431122567,-0.1467286321,2.5561122694$ C, $3.7424147016,-0.2293266395,2.2724923208$ $\mathrm{H}, 4.4178495898,-0.8424140007,2.8646577379$ $\mathrm{H}, 4.1824322582,0.3322106279,1.4520630063$ C, $1.861054683,-0.8891388547,3.7366934503$ $\mathrm{H}, 1.3671939591,-0.1937528885,4.4283016034$ $\mathrm{H}, 1.101401038,-1.6090810052,3.4124761134$ $\mathrm{H}, 2.634611371,-1.42705352,4.2921199048$ 


\section{$\mathrm{R}=$ Mesityl, $\mathrm{H}=-1295.136815$}

$\mathrm{Rh},-0.5869219925,0.0116763773,0.0533791537$ $\mathrm{H},-0.2249993211,-1.3648715715,0.6378863673$ $\mathrm{N}, 0.803490298,-0.2843972892,-1.4975595765$ $\mathrm{N}, 0.4635082572,0.0691197401,-2.7572076263$ $\mathrm{N},-2.0222748645,-0.8200697307,-1.3592272701$ $\mathrm{N},-1.9945079155,-0.3655316085,-2.6285858847$ $\mathrm{N},-1.0035609904,1.9522166403,-0.9135464318$ $\mathrm{N},-1.1094068064,1.9652401472,-2.2575763937$ $\mathrm{N},-2.5626935117,0.2205202861,2.3626871528$ C, $2.0141381818,-0.8425728969,-1.5527698988$ C, $2.4798409609,-0.8477750899,-2.8739364137$ $\mathrm{H}, 3.4212377962,-1.2263658483,-3.2423427482$ C, $1.4593373138,-0.2612403673,-3.6040930612$ $C,-3.0007319132,-1.7237050622,-1.2867350495$ $C,-3.6269708059,-1.8628958824,-2.5333296667$ $\mathrm{H},-4.4465554837,-2.5145387,-2.7965388566$ C, $-2.9496864584,-0.9795140158,-3.3576045097$ $C_{,}-1.1840341635,3.2056338748,-0.4952744003$ C, $-1.4132382821,4.0559238319,-1.5867904689$ $\mathrm{H},-1.5918028644,5.1206459703,-1.5746599795$ C, $-1.3550513742,3.220025219,-2.6895749443$ C, $0.8899936095,0.8906300781,1.2517185442$ $\mathrm{H}, 0.3851820764,1.5635791017,1.9561263527$ $\mathrm{C},-1.7985982855,0.1465825308,1.4728501968$ C, $-3.4876212983,0.2883465286,3.4247464715$ $\mathrm{H},-4.444852686,0.6783181837,3.066014515$ $\mathrm{H},-3.1103267597,0.9490094504,4.2110284978$ $\mathrm{B},-0.9281225105,0.6640674998,-3.0655421361$ $\mathrm{H},-1.0306695953,0.8909012605,-4.2426841063$ $\mathrm{H},-3.0739626536,-0.7467383566,-4.4052739091$ $\mathrm{H},-3.1974841375,-2.2303966498,-0.3524320049$ $\mathrm{H}, 1.3672311515,-0.0562403769,-4.6607538221$ $\mathrm{H}, 2.479532558,-1.2003285648,-0.6454593583$ $\mathrm{H},-1.467872605,3.4250125153,-3.7443167673$ $\mathrm{H},-1.1338743492,3.4349266812,0.5601754759$ $\mathrm{H},-3.6485160912,-0.7072866328,3.8494377348$ $\mathrm{H}, 1.4505189104,1.5176131153,0.5499254283$ C, $1.8280993531,-0.0002090569,2.0046688856$ C, $1.3924587631,-0.7778719496,3.0925493501$ C, $2.262736892,-1.5873562157,3.81613639$ C, $3.6154200228,-1.6255882006,3.4448141182$ C, $4.0906814685,-0.8638815486,2.379824766$ C, $3.1865321104,-0.0574636857,1.6735090841$ $\mathrm{H}, 0.3389489234,-0.7545890104,3.364105726$ $\mathrm{H}, 4.3056183354,-2.257792986,4.0012980575$ $\mathrm{H}, 3.5536675603,0.5559300756,0.8518689198$ C, $5.5503105384,-0.8795844823,2.0033197755$ $\mathrm{H}, 6.03380469,0.0741602313,2.2476424563$ $\mathrm{H}, 6.0922534222,-1.6692697067,2.5317334295$ $\mathrm{H}, 5.6864879333,-1.0400288741,0.9280880337$ C, $1.7716847417,-2.4182380639,4.9740793245$ $\mathrm{H}, 2.3111407947,-2.1774478541,5.8976024709$ $\mathrm{H}, 0.7055645042,-2.2564620547,5.1580309039$ $\mathrm{H}, 1.9181405907,-3.4886744461,4.7876071986$

\section{$\mathrm{R}=$ Me-vinyl, $\mathrm{H}=-1063.040072$}

$\mathrm{Rh},-0.2354785287,-0.4110857035,-0.6019434014$ $\mathrm{H},-0.5835796313,-1.8564958512,-1.0020260598$ $\mathrm{N},-1.0807207983,-0.7174121689,1.2965486816$ $\mathrm{N},-0.4359395112,-0.2600126766,2.3911755715$ $\mathrm{N}, 1.6456153091,-0.9709833727,0.349154109$ $\mathrm{N}, 1.9203852371,-0.4513095267,1.5632887544$ $\mathrm{N}, 0.2071098844,1.6241563735,0.1320665571$
$\mathrm{N}, 0.695131289,1.7421856009,1.3838522002$ $\mathrm{N}, 0.8520457942,-0.1332119839,-3.4344207804$ $C,-2.1982034664,-1.3170098368,1.7094731687$ $C,-2.2873333269,-1.2513869451,3.1058925645$ $\mathrm{H},-3.0692384103,-1.643040299,3.7387300221$ C, $-1.144761977,-0.5710858685,3.4953069829$ $C, 2.6664337914,-1.765430623,0.023234536$ C, $3.629057051,-1.7642190035,1.0423750109$ $\mathrm{H}, 4.5625098468,-2.3056416854,1.0758433656$ C, $3.1085565139,-0.9142402272,2.0041667727$ C, $0.1059691471,2.8565516808,-0.3669999717$ C, $0.5378306256,3.8006700916,0.5759915875$ $\mathrm{H}, 0.5760504499,4.8747215584,0.4727299145$ C, $0.9026883044,3.0431734053,1.6766855402$ $C,-2.0473665021,0.1091288774,-1.3009486857$ $\mathrm{H},-2.4236932711,1.0267931071,-0.8303405174$ C, $-2.8496843234,-0.4942080205,-2.1889789484$ $\mathrm{H},-2.5200054375,-1.4134821125,-2.6771671817$ C, $0.4413956541,-0.2326607478,-2.3397316288$ C, $1.2880975176,-0.0090969789,-4.7691045614$ $\mathrm{H}, 2.248217717,0.5140672164,-4.8047280359$ $\mathrm{H}, 0.5546860284,0.5565581355,-5.351400958$ $B, 0.909721264,0.4876677832,2.2579805303$ $\mathrm{H}, 1.3171713639,0.8042435877,3.344776337$ $\mathrm{H}, 3.4931774758,-0.6082996094,2.9662722838$ $\mathrm{H}, 2.6544446988,-2.2991677222,-0.9166907825$ $\mathrm{H},-0.785163414,-0.2857720141,4.4732344712$ $\mathrm{H},-2.8702228308,-1.7484066482,0.9820561616$ $\mathrm{H}, 1.2906000505,3.3332621021,2.6424523672$ $\mathrm{H},-0.2750848773,3.0018499254,-1.3684639426$ $\mathrm{H}, 1.4084301247,-0.9989268282,-5.2197310413$ C, $-4.2188868712,-0.0051671643,-2.5759727415$ $\mathrm{H},-4.480021302,0.9117071759,-2.0368650973$ $\mathrm{H},-4.2859410829,0.2067556391,-3.6518412624$ $\mathrm{H},-4.9925532887,-0.7542054936,-2.359283041$

\section{$\mathrm{R}=$ Vinyl, $\mathrm{H}=-1023.763222$}

$\mathrm{Rh},-0.7112837511,-0.4145753243,-0.4067784804$ $\mathrm{H},-1.1119118835,-0.7571279772,-1.8532447887$ $\mathrm{N}, 1.1828869449,-1.2644618892,-0.7170372593$ $\mathrm{N}, 2.2804879894,-0.6231337864,-0.2619137604$ $\mathrm{N}, 0.244784442,1.4694242395,-0.9593419894$ $\mathrm{N}, 1.4610601775,1.7378611754,-0.4416500566$ $\mathrm{N}, 0.0257261298,0.0223838066,1.6282516935$ $\mathrm{N}, 1.2799226028,0.5044109222,1.7460307534$ $\mathrm{N},-3.5447841247,0.6769952075,-0.1374493708$ C, $1.5907469693,-2.3822047524,-1.3199634226$ C, $2.9870118361,-2.4750090206,-1.2585754873$ $\mathrm{H}, 3.61647012,-3.2580353167,-1.6533750553$ C, $3.3815630303,-1.3346723808,-0.5774811651$ C, $-0.0810140227,2.4957122405,-1.7466627222$ C, $0.9404508018,3.4558116029,-1.7424829353$ $\mathrm{H}, 0.9746795402,4.392678695,-2.2779120582$ C, $1.9033909362,2.9277998824,-0.8984187957$ C, $-0.473199191,-0.0775187615,2.8609263536$ C, $0.4724016098,0.348493852,3.8049296047$ $\mathrm{H}, 0.3699139025,0.3858265762,4.8790739466$ C, $1.5743403416,0.7090075133,3.0471280442$ C, $-1.4154851834,-2.2228917315,0.1048451221$ $\mathrm{H},-0.9582357051,-2.5964288252,1.0286642389$ C, $-2.2939792211,-3.0209037891,-0.5139731518$ $\mathrm{H},-2.7913163569,-2.7361614634,-1.4392368392$ C, $-2.4499454255,0.2663693338,-0.2318849438$ $C,-4.8827462563,1.1061665175,-0.0235279775$ 
$\mathrm{H},-4.9337154741,2.0298810338,0.5603058745$ $\mathrm{H},-5.4801986849,0.3374880675,0.475449339$ $\mathrm{B}, 2.1533168245,0.7202602747,0.4908995042$ $\mathrm{H}, 3.2418580298,1.1226443514,0.8076617545$ $\mathrm{H}, 2.8669513579,3.3087885073,-0.5924540489$ $\mathrm{H},-1.0225130295,2.4889587499,-2.2776290884$ $\mathrm{H}, 4.3613237863,-0.9784909626,-0.2942240646$ $\mathrm{H}, 0.8597909418,-3.0515884012,-1.7496700766$ $\mathrm{H}, 2.5422224984,1.0916102954,3.3371671233$ $\mathrm{H},-1.4769201092,-0.4524087459,3.0067003713$ $\mathrm{H},-5.3043268278,1.2919181168,-1.0159981061$ $\mathrm{H},-2.5446939124,-4.0062852501,-0.1181256385$

\section{$\mathrm{R}=\mathrm{Et}, \mathrm{H}=-1024.973573$}

$\mathrm{Rh},-0.3433398905,-0.5473234495,-0.6130860559$ $\mathrm{H},-0.2408793887,-2.0561188813,-0.9156160295$ $\mathrm{N},-0.7619401048,-0.9781033304,1.4031400422$ $\mathrm{N},-0.1694266747,-0.2485595737,2.3725064293$ $\mathrm{N}, 1.7399869189,-0.3941935332,0.070381919$ $\mathrm{N}, 1.9812258296,0.2875530623,1.2085390904$ $\mathrm{N},-0.5063693216,1.5691364789,-0.0133518261$ $\mathrm{N}, 0.0733608528,1.9325126702,1.1491423693$ $\mathrm{N}, 0.2468025813,-0.1525594091,-3.5733399364$ C, $-1.5186249154,-1.8983214763,2.0043053553$ C, $-1.4215138436,-1.7674899681,3.395263072$ $\mathrm{H},-1.9115047293,-2.3604593996,4.1525589049$ C, $-0.5510677414,-0.7058862087,3.5820600527$ C, $2.9168272889,-0.8301419536,-0.3808073513$ C, $3.9489822699,-0.4241137745,0.4773823244$ $\mathrm{H}, 5.006593731,-0.6210581542,0.3857673098$ C, $3.3037937335,0.2837024871,1.4777190269$ $C,-1.0667494361,2.6638997395,-0.528629883$ C, $-0.8502917372,3.7654189798,0.3119689096$ $\mathrm{H},-1.1792082317,4.7841404385,0.1716230739$ C, $-0.1198200949,3.2500047318,1.3695108609$ $C,-2.3607685122,-0.6614707007,-1.0793888604$ $\mathrm{H},-2.6262889239,0.2492208344,-1.6344488313$ C, $0.0179988184,-0.2971305001,-2.429484664$ C, $0.5331476807,-0.006133007,-4.9456291611$ $\mathrm{H}, 1.2631784595,0.795067635,-5.0953744557$ $\mathrm{H},-0.3789059874,0.2414341983,-5.4971572896$ B, $0.8119491855,0.8910685831,2.0155444834$ $\mathrm{H}, 1.225623608,1.4085245154,3.020014973$ $\mathrm{H}, 3.6861349929,0.7800853924,2.3578994638$ $\mathrm{H}, 2.9616205708,-1.4156923268,-1.2883751483$ $\mathrm{H},-0.1766052084,-0.2434285284,4.4836296928$ $\mathrm{H},-2.0866533562,-2.5988240529,1.4094576422$ $\mathrm{H}, 0.2732257862,3.7208065763,2.2589465927$ $\mathrm{H},-1.5985872903,2.6069673876,-1.4681702774$ $\mathrm{H}, 0.94418131,-0.9375358592,-5.3472451139$ $\mathrm{H},-2.8732583838,-0.5917364215,-0.1117676955$ C, $-2.8573922338,-1.8840217383,-1.8449287818$ $\mathrm{H},-2.3724625199,-1.9789244409,-2.8233099965$ $\mathrm{H},-2.6559799387,-2.8137568535,-1.3013378902$ $\mathrm{H},-3.9422835854,-1.8349039676,-2.0211168431$

\section{$\mathrm{R}=\mathrm{Pr}, \mathrm{H}=-1064.246416$}

$\mathrm{Rh},-0.1493772724,-0.4629241049,-0.5447767505$ $\mathrm{H},-0.0516534392,-1.9667171114,-0.8746301339$ $\mathrm{N},-0.6352669106,-0.9252770939,1.449165955$ $\mathrm{N},-0.0650673242,-0.2188365209,2.4487004039$ $\mathrm{N}, 1.9097968511,-0.3489754732,0.2023710373$ $\mathrm{N}, 2.1241756314,0.3126460355,1.3578427158$ $\mathrm{N},-0.3128943837,1.642329046,0.0878073441$
$\mathrm{N}, 0.2361593616,1.9802121651,1.2725658223$ $\mathrm{N}, 0.5353253207,-0.0174975936,-3.4771380895$ $C,-1.4201320698,-1.8466754381,2.0110207964$ $C,-1.3644076484,-1.7404184664,3.4063758201$ $\mathrm{H},-1.8838133673,-2.3404097691,4.1381091281$ C, $-0.488616976,-0.6921171478,3.6379628991$ $C, 3.0946317804,-0.7931249268,-0.2191300744$ C, $4.1046548323,-0.4132276893,0.6764986856$ $\mathrm{H}, 5.1620224437,-0.6225614677,0.6142714316$ C, $3.437581247,0.2876272903,1.6673781581$ $C,-0.8499533898,2.7505528107,-0.4235313138$ C, $-0.6485089174,3.8353106755,0.4422779545$ $\mathrm{H},-0.9652541162,4.8590669792,0.310861312$ C, $0.0474876189,3.2952781043,1.5107459139$ $C,-2.1537525763,-0.5450440941,-1.0711671524$ $\mathrm{H},-2.3921791528,0.368044372,-1.637047304$ C, $0.2701481186,-0.1817445719,-2.3439290678$ C, $0.8581219639,0.1510733448,-4.8387759157$ $\mathrm{H}, 1.5522846876,0.987933303,-4.9612822499$ $\mathrm{H},-0.047048148,0.3568015162,-5.4181253088$ B, $0.9386047135,0.9162784088,2.1413565047$ $\mathrm{H}, 1.3282891832,1.4110002168,3.1666272801$ $\mathrm{H}, 3.7982115256,0.765258243,2.5668582086$ $\mathrm{H}, 3.1603854907,-1.3655071845,-1.1338118262$ $\mathrm{H},-0.1371450948,-0.2491145399,4.5583793989$ $\mathrm{H},-1.9774534736,-2.5305299153,1.3874010219$ $\mathrm{H}, 0.4188998825,3.7472307389,2.419072346$ $\mathrm{H},-1.3566521573,2.7142537333,-1.3778906092$ $\mathrm{H}, 1.327131583,-0.7566369975,-5.230980723$ $\mathrm{H},-2.695255074,-0.4656587655,-0.1188687941$ C, $-2.6570012421,-1.759202408,-1.8489573049$ $\mathrm{H},-2.1055303557,-1.8552392325,-2.7939333771$ $\mathrm{H},-2.4388455864,-2.6776982069,-1.2880999616$ C, $-4.157848862,-1.6950336477,-2.1470100656$ $\mathrm{H},-4.4042999728,-0.8097604809,-2.7455042955$ $\mathrm{H},-4.5015465135,-2.5771338705,-2.7000458613$ $\mathrm{H},-4.7416177533,-1.6341558512,-1.2209507553$

\section{$\mathrm{R}=\mathrm{CH}_{2} \mathrm{SiMe}_{3}, \mathrm{H}=-1108.638701$}

$\mathrm{Rh},-0.1177695136,-0.2168256573,0.2609466833$ $\mathrm{H}, 0.2546031311,-1.6632031839,0.6432056146$ $\mathrm{N}, 0.9019470823,-0.3979073953,-1.5734438519$ $\mathrm{N}, 0.3244760477,0.0954967325,-2.6912320942$ $\mathrm{N},-1.8611293686,-0.8319564741,-0.8798285389$ $\mathrm{N},-2.0745306572,-0.253005009,-2.0786199744$ $\mathrm{N},-0.5881691817,1.8318844963,-0.4157148898$ $\mathrm{N},-0.9876614469,1.988797107,-1.6939455481$ $\mathrm{N},-1.6133809486,-0.0763472497,2.9087618265$ $C, 2.0627483005,-0.9473509302,-1.9365523134$ C, $2.2506459016,-0.8087231239,-3.3172469543$ $\mathrm{H}, 3.0871008908,-1.1481901845,-3.909108771$ C, $1.1188587003,-0.1407511005,-3.7544973688$ $C,-2.8544778865,-1.699872848,-0.680425012$ $C,-3.7362770353,-1.687651038,-1.7702177696$ $\mathrm{H},-4.6303010526,-2.2769084376,-1.9084227363$ $C,-3.1964196202,-0.7524921608,-2.6376618855$ C, $-0.5990314878,3.0399453122,0.1482110903$ C, $-1.0133197049,4.0072993695,-0.7790809453$ $\mathrm{H},-1.1216259208,5.070766818,-0.6273891485$ C, $-1.2485204981,3.2899404731,-1.9401340483$ C, $1.6314950307,0.407016106,1.216276082$ $\mathrm{H}, 1.3972126805,1.3046841071,1.809022548$ C, $-1.0213940265,-0.131443537,1.8961763965$ C, $-2.261973867,-0.0703038838,4.1604534078$ 
$\mathrm{H},-1.6363547587,-0.570965254,4.905628852$ $\mathrm{H},-3.2202546168,-0.5937319861,4.0912161817$ $\mathrm{B},-1.0636175992,0.7718954353,-2.6380701655$ $\mathrm{H},-1.3959595503,1.1215650118,-3.7401355813$ $\mathrm{H},-3.5244666771,-0.4096840294,-3.6082407879$ $\mathrm{H},-2.8794815952,-2.2908390203,0.2242525499$ $\mathrm{H}, 0.8207273425,0.1830121584,-4.7410472202$ $\mathrm{H}, 2.6925842971,-1.4124869376,-1.1934765781$ $\mathrm{H},-1.5774697249,3.60835972,-2.9187593927$ $\mathrm{H},-0.3043419154,3.1556731044,1.1819393514$ $\mathrm{H},-2.4470322841,0.9579671892,4.4849450968$ $\mathrm{H}, 2.2891504516,0.7504733025,0.4055933473$ $\mathrm{Si}, 2.5954896397,-0.77219214,2.3285961369$ C, $1.5500300838,-1.4092657765,3.7870668281$ $\mathrm{H}, 2.1560440868,-2.0311723139,4.4550984276$ $\mathrm{H}, 1.1525354083,-0.5768813193,4.3787447825$ $\mathrm{H}, 0.7089139764,-2.0133538536,3.4326808671$ C, $3.2748679468,-2.2855819168,1.3961591151$ $\mathrm{H}, 4.0263482519,-1.9928517818,0.6540282245$ $\mathrm{H}, 3.7602258856,-2.9801147952,2.090783473$ $\mathrm{H}, 2.4740990525,-2.8275999096,0.8826351693$ C, $4.0903646469,0.1549252344,3.070530787$ $\mathrm{H}, 3.7662280907,1.0157973909,3.6658855016$ $\mathrm{H}, 4.6856438526,-0.4955933464,3.7213063837$ $\mathrm{H}, 4.7502922558,0.530768381,2.2808350443$

\section{$\mathrm{R}=i-\mathrm{Pr}, \mathrm{H}=-1064.240275$}

$\mathrm{Rh},-0.6990183891,-0.2187031379,-0.422367473$ $\mathrm{H},-1.1133282289,-0.4670881499,-1.887658771$ $\mathrm{N}, 1.1266808078,-1.2115281913,-0.8016375264$ $\mathrm{N}, 2.2615173521,-0.722685845,-0.2542544759$ $\mathrm{N}, 0.4886564095,1.5787108017,-0.9021956927$ $\mathrm{N}, 1.6981924528,1.7075618063,-0.3205963226$ $\mathrm{N}, 0.0268527761,0.0836272594,1.6363079492$ $\mathrm{N}, 1.3260879013,0.4043684338,1.8010777037$ $\mathrm{N},-3.3460680791,1.266743267,-0.2006149237$ C, $1.4765893849,-2.2287548203,-1.5912542707$ C, $2.8634307171,-2.4180537765,-1.5523827154$ $\mathrm{H}, 3.4459359434,-3.1620315399,-2.0742534983$ C, $3.3205791468,-1.4335609902,-0.6921479841$ C, $0.2972323116,2.6665359317,-1.6496611194$ C, $1.4023817421,3.5247905896,-1.5547728968$ $\mathrm{H}, 1.549159199,4.4769491467,-2.0422278718$ C, $2.2714507244,2.869621798,-0.6982596937$ C, $-0.5194727416,0.0322086345,2.8520421847$ C, $0.4405818088,0.3246183674,3.8315171477$ $\mathrm{H}, 0.3087362835,0.3600998596,4.9025112192$ C, $1.6021637941,0.5555308849,3.1135796941$ C, $-1.7518604864,-1.9607028883,0.1114027968$ $\mathrm{H},-2.4519212674,-1.6248904815,0.8920513924$ C, $-2.3235647643,0.6917090098,-0.2727711585$ C, $-4.5718432137,1.9612291896,-0.152883321$ $\mathrm{H},-4.4365507128,2.9324415462,0.3325174386$ $\mathrm{H},-5.3096455933,1.3846989066,0.413144199$ $\mathrm{B}, 2.2496180731,0.5790259725,0.5789719829$ $\mathrm{H}, 3.3657694585,0.845503009,0.9411575428$ $\mathrm{H}, 3.2518640381,3.1413972527,-0.334570658$ $\mathrm{H},-0.6144488318,2.7736171427,-2.2207194807$ $\mathrm{H}, 4.3186665748,-1.1839919309,-0.3628987683$ $\mathrm{H}, 0.718575293,-2.7693190066,-2.138961502$ $\mathrm{H}, 2.599516017,0.814581733,3.4381488498$ $\mathrm{H},-1.565171864,-0.2191544202,2.9635718553$ $\mathrm{H},-4.9541634196,2.1225901228,-1.165585455$ C, $-2.5854760229,-2.5714329532,-1.0148488324$
$\mathrm{H},-3.2998098163,-1.8564949195,-1.4356854117$ $\mathrm{H},-1.9532863741,-2.9192972654,-1.8404873059$ $\mathrm{H},-3.1555925569,-3.4427516952,-0.6548606887$ $C,-0.8632415059,-3.0248113105,0.7546086354$ $\mathrm{H},-0.2477029901,-2.6188412305,1.5622734083$ $\mathrm{H},-1.4859882307,-3.8269936461,1.1805618758$ $\mathrm{H},-0.1900184944,-3.4956618118,0.0320302179$

\section{$\mathrm{R}=t-\mathrm{Bu}, \mathrm{H}=-1103.506614$}

$\mathrm{Rh},-0.6187075334,-0.1011969083,-0.4625479942$ $\mathrm{H},-0.9811200434,-0.281197125,-1.9461835217$ $\mathrm{N}, 1.0756571494,-1.350647267,-0.7546989788$ $\mathrm{N}, 2.2403927349,-1.0131592784,-0.1567557591$ $\mathrm{N}, 0.8415752014,1.5048449793,-0.8943267023$ $\mathrm{N}, 2.0371329608,1.4683755922,-0.2744648691$ $\mathrm{N}, 0.0667005608,0.1527689918,1.6428128398$ $\mathrm{N}, 1.3949779753,0.2791974608,1.8443528786$ $\mathrm{N},-2.8596374478,1.9529994035,-0.4459273777$ C, $1.3278732953,-2.4081512628,-1.5300462671$ C, $2.674391744,-2.7768851885,-1.4298236917$ $\mathrm{H}, 3.1768164554,-3.5924824029,-1.9273086368$ C, $3.2165489242,-1.8569387501,-0.5487117227$ C, $0.8355522363,2.5913593207,-1.6681782701$ C, $2.0515425001,3.2800537255,-1.550601566$ $\mathrm{H}, 2.3524746393,4.1890565102,-2.0495330736$ C, $2.7872651816,2.524298384,-0.6526495069$ $C,-0.514661673,0.2613654282,2.8393939839$ C, $0.4478427959,0.4573162899,3.8396180349$ $\mathrm{H}, 0.2894328527,0.5739991413,4.9011777704$ C, $1.6516776627,0.4628720087,3.1566129129$ C, $-1.9510743264,-1.7315614881,-0.0714277133$ C, $-2.031852889,1.1189623394,-0.421569846$ $C,-3.897756635,2.9059095538,-0.43312766$ $\mathrm{H},-3.5721079446,3.8108496875,0.0885297468$ $\mathrm{H},-4.7754366399,2.4997941957,0.0795824612$ $B, 2.3793079084,0.2888580075,0.6616739915$ $\mathrm{H}, 3.5068794149,0.3972131243,1.0678882517$ $\mathrm{H}, 3.7849025107,2.6577494067,-0.2598681828$ $\mathrm{H},-0.0320351102,2.8178980135,-2.2719748261$ $\mathrm{H}, 4.2229476047,-1.7370417209,-0.1750970409$ $\mathrm{H}, 0.5350648275,-2.8518333049,-2.1129988191$ $\mathrm{H}, 2.6672039268,0.5837510258,3.5047962227$ $\mathrm{H},-1.5888047929,0.1873691382,2.9289344177$ $\mathrm{H},-4.1799623841,3.1716819413,-1.4564862149$ C, $-2.4014792533,-2.4017722133,-1.3744089341$ $\mathrm{H},-2.9708861093,-1.713204098,-2.0074613792$ $\mathrm{H},-1.561839703,-2.7735011889,-1.9692083426$ $\mathrm{H},-3.0497450955,-3.2672153215,-1.155280568$ C, $-1.2386078406,-2.7663269385,0.8073152881$ $\mathrm{H},-0.9178679194,-2.340026064,1.7622870551$ $\mathrm{H},-1.9268278413,-3.5979070262,1.0313829308$ $\mathrm{H},-0.3583368712,-3.1949321987,0.323636461$ C, $-3.2197351437,-1.2940701089,0.6732764761$ $\mathrm{H},-3.8534823289,-0.6422286492,0.0628002034$ $\mathrm{H},-3.8258935489,-2.1762713217,0.9360879989$ $\mathrm{H},-2.9988298571,-0.7711967005,1.6096135401$

\section{$\mathrm{R}=c-\mathrm{Pr}, \mathrm{H}=-1063.024228$}

$\mathrm{Rh}, 0.673526666,0.2102050195,-0.3868204154$ $\mathrm{H}, 1.139802286,0.5594700066,-1.8156749393$ $\mathrm{N},-1.050652158,1.3990988068,-0.6069882448$ $\mathrm{N},-2.2359472351,0.9207991398,-0.1673791766$ $\mathrm{N},-0.6057065042,-1.4289993794,-1.0524982764$ $\mathrm{N},-1.8412712239,-1.5259006154,-0.5211153331$ 
$\mathrm{N},-0.1130912132,-0.2225292213,1.6267346868$ $\mathrm{N},-1.4285532621,-0.4953963851,1.7403757549$ $\mathrm{N}, 3.2134705664,-1.4577056215,-0.1996093691$ C, $-1.2789285542,2.6018480152,-1.1362980975$ C, $-2.6398091957,2.9190549772,-1.0407570901$ $\mathrm{H},-3.1356017184,3.8171422042,-1.376771767$ $C,-3.2086072188,1.8201691656,-0.4182061738$ C, $-0.4737424818,-2.4319420119,-1.9220496399$ C, $-1.6451945426,-3.2015116242,-1.959039$ $\mathrm{H},-1.8485749107,-4.0742611164,-2.5611969212$ C, $-2.4894892686,-2.5839905662,-1.0511833887$ C, $0.4088227847,-0.293601204,2.8519915014$ C, $-0.5848069679,-0.6202403737,3.7863540651$ $\mathrm{H},-0.4784889734,-0.7487035573,4.8530763067$ C, $-1.7398024321,-0.7377413598,3.0309658383$ C, $2.2331107515,-0.8149041116,-0.2658591416$ C, $4.4112799561,-2.1956172506,-0.1138824578$ $\mathrm{H}, 4.2338663797,-3.1438334642,0.4022308391$ $\mathrm{H}, 5.163776916,-1.6268716223,0.4406345264$ $\mathrm{B},-2.3367613344,-0.4722783601,0.4931259395$ $\mathrm{H},-3.475979181,-0.7014052077,0.8050389298$ $\mathrm{H},-3.4999695013,-2.8160649457,-0.7474220307$ $\mathrm{H}, 0.4461057721,-2.5442208444,-2.4786013495$ $\mathrm{H},-4.2301885591,1.6122327001,-0.1350957475$ $\mathrm{H},-0.4572028175,3.1702555809,-1.5445805071$ $\mathrm{H},-2.7551936982,-0.9725199066,3.3158092357$ $\mathrm{H}, 1.4625221172,-0.1021710641,3.0007605277$ $\mathrm{H}, 4.7972453136,-2.4066343153,-1.1159005533$ C, $1.7119637223,1.8523331066,0.2572142566$ C, $3.1627225064,2.1863814744,0.0154936897$ C, $2.0838409161,3.0402371843,-0.5989075809$ $\mathrm{H}, 1.3591383938,2.1291124255,1.2530030029$ $\mathrm{H}, 3.7414371665,1.5605806525,-0.6592866828$ $\mathrm{H}, 3.7494874361,2.607975601,0.829761852$ $\mathrm{H}, 1.9335292726,4.0419868866,-0.2000823531$ $\mathrm{H}, 1.9534923684,2.9716763149,-1.6760743485$

\section{$\mathrm{R}=c-\mathrm{Bu}, \mathrm{H}=-1102.297170$}

$\mathrm{Rh}, 0.0957014844,-0.4195073871,0.5560143549$ $\mathrm{H}, 0.4201128725,-1.8691597285,0.9797339019$ $\mathrm{N}, 1.2596591391,-0.6506925573,-1.1942428191$ $\mathrm{N}, 0.8047818826,-0.1132966839,-2.3494932089$ $\mathrm{N},-1.5723495458,-0.9850763333,-0.7753484632$ $\mathrm{N},-1.6518915565,-0.3802538089,-1.9778140749$ $\mathrm{N},-0.2804464577,1.6366432953,-0.1492164601$ $\mathrm{N},-0.5372570972,1.8197019799,-1.4594048243$ $\mathrm{N},-1.6587330818,-0.3224339759,3.0409996044$ C, $2.3787627193,-1.3172707098,-1.4857075594$ C, $2.6681197857,-1.2070501653,-2.8511025602$ $\mathrm{H}, 3.5046164521,-1.6291513433,-3.3870990358$ C, $1.6371708533,-0.4354249481,-3.3599298565$ $C,-2.6094315659,-1.8191577095,-0.6888369528$ $C,-3.3834329867,-1.7586579322,-1.8568612207$ $\mathrm{H},-4.2798844995,-2.3132333049,-2.0905341283$ C, $-2.7319425719,-0.8295939075,-2.6509628455$ $C_{,}-0.3218583124,2.8381670921,0.4287435744$ C, $-0.6123389329,3.826833677,-0.5225892592$ $\mathrm{H},-0.7118356701,4.8906624606,-0.3674367661$ C, $-0.7404226384,3.1295963186,-1.7124370879$ C, $-0.9668502322,-0.3457070148,2.0918609419$ C, $-2.4546567572,-0.3027741477,4.2037529451$ $\mathrm{H},-3.3352357395,0.3275426746,4.0477203964$ $\mathrm{H},-1.8801532418,0.0951982765,5.0458363762$ $B,-0.5568378964,0.6148323242,-2.4198639785$
$\mathrm{H},-0.7654006951,0.9870464942,-3.5450365445$ $\mathrm{H},-2.953803664,-0.4602604493,-3.6417758258$ $\mathrm{H},-2.7420940141,-2.4223746123,0.1984230038$ $\mathrm{H}, 1.432357273,-0.0944906576,-4.3642833434$ $\mathrm{H}, 2.9127180253,-1.8380948167,-0.7062950554$ $\mathrm{H},-0.9579028077,3.4677578073,-2.7152445637$ $\mathrm{H},-0.1371829777,2.9333322228,1.4898457097$ $\mathrm{H},-2.7862563622,-1.3157387373,4.4519736969$ C, $1.732653833,0.1615051934,1.6755874431$ C, $1.8074898973,0.1103466997,3.2245222295$ C, $3.334857311,-0.0678267815,3.0983673087$ C, $3.1003257339,-0.5749271241,1.6575513287$ $\mathrm{H}, 1.9103134659,1.2004440936,1.3609354861$ $\mathrm{H}, 1.3488531002,-0.8034206686,3.620055342$ $\mathrm{H}, 1.4265730811,0.9672608152,3.7938327449$ $\mathrm{H}, 3.8372718465,-0.7377551983,3.8054538829$ $\mathrm{H}, 3.8529215471,0.8973662941,3.100795307$ $\mathrm{H}, 2.9746645956,-1.6642949212,1.6510336772$ $\mathrm{H}, 3.8299503042,-0.2954605542,0.8906308061$

\section{$\mathrm{R}=$ Allyl, $\mathrm{H}=-1063.034596$}

$\mathrm{Rh}, 0.7374158039,0.1193030692,-0.2609997259$ $\mathrm{H}, 1.3300022011,0.5827794558,-1.6064373754$ $\mathrm{N},-0.8379649465,1.4913082497,-0.4657713656$ $\mathrm{N},-2.0955206746,1.1013914691,-0.1625036332$ $\mathrm{N},-0.6283688978,-1.2867908755,-1.2103665933$ $\mathrm{N},-1.9044905238,-1.316382972,-0.7752572941$ $\mathrm{N},-0.2393898249,-0.4532171585,1.630474695$ $\mathrm{N},-1.5804366439,-0.5950461116,1.617201278$ $\mathrm{N}, 3.1203598681,-1.7776557991,-0.1646107158$ C, $-0.9011958321,2.7517189913,-0.8973934027$ $C,-2.2286588519,3.1988646388,-0.8717080903$ $\mathrm{H},-2.6056757442,4.1692361942,-1.1572710779$ C, $-2.9518958607,2.1155181058,-0.399934271$ C, $-0.523043687,-2.1964956345,-2.1802479142$ C, $-1.7542405159,-2.8356849694,-2.3829089812$ $\mathrm{H},-1.9910530385,-3.6118972664,-3.094981447$ C, $-2.6047657984,-2.2395766484,-1.4665279151$ C, $0.1696273559,-0.720280868,2.8714027073$ C, $-0.9246310184,-1.0440631035,3.6865106779$ $\mathrm{H},-0.9195654131,-1.3049650702,4.7341573114$ C, $-2.0191444113,-0.9497912754,2.8431082243$ C, $1.8827221304,1.5784411986,0.7057064825$ $\mathrm{H}, 2.5348622099,1.057609606,1.4195730365$ C, $2.1975554109,-1.0506763992,-0.1925730694$ C, $4.2245092987,-2.6544686399,-0.1693295223$ $\mathrm{H}, 3.9029908024,-3.6663572178,0.0947977911$ $\mathrm{H}, 4.9728871031,-2.3219921038,0.5561743207$ $\mathrm{B},-2.3789609847,-0.3370853608,0.3220985801$ $\mathrm{H},-3.5557010655,-0.4834341281,0.5252402818$ $\mathrm{H},-3.6525207477,-2.4014242001,-1.2586349692$ $\mathrm{H}, 0.4222689525,-2.3386333445,-2.6848175308$ $\mathrm{H},-4.009200088,1.9914877032,-0.2160490287$ $\mathrm{H}, 0.0069138206,3.2597780795,-1.1914809358$ $\mathrm{H},-3.0725747519,-1.1060794768,3.0250128966$ $\mathrm{H}, 1.2206528047,-0.6613925818,3.1181716847$ $\mathrm{H}, 4.6836755703,-2.6771354887,-1.1624179215$ $\mathrm{H}, 1.1664107677,2.1769553759,1.2804004376$ C, $2.6968442219,2.4386043812,-0.1871943391$ C, $2.560952156,3.7619111266,-0.3502845542$ $\mathrm{H}, 3.4767982609,1.9309557593,-0.758940642$ $\mathrm{H}, 3.1976066692,4.323614924,-1.028584267$ $\mathrm{H}, 1.8151893516,4.32830943,0.2046614057$

$\mathrm{R}=$ Benzyl, $\mathrm{H}=-1216.583531$ 
$\mathrm{Rh},-0.30617621,-0.1101193403,0.2902274527$ $\mathrm{H}, 0.076462015,-1.4865423544,0.8612573964$ $\mathrm{N}, 1.0673218827,-0.3816468022,-1.2789814245$ $\mathrm{N}, 0.7096239937,-0.0205677471,-2.5315167227$ $\mathrm{N},-1.7482074787,-0.9463433771,-1.1095787311$ $\mathrm{N},-1.7424965045,-0.4798806132,-2.3749006425$ $\mathrm{N},-0.7529518433,1.8343417732,-0.657110555$ $\mathrm{N},-0.8760770317,1.8568868856,-1.999541017$ $\mathrm{N},-2.2645570838,0.0579770773,2.6182938202$ C, $2.2810083467,-0.9311968139,-1.3532848338$ C, $2.7307039015,-0.9227357168,-2.679830794$ $\mathrm{H}, 3.6700991899,-1.2920254706,-3.0624488451$ C, $1.6973594615,-0.3375997653,-3.3928838163$ $C_{,}-2.715973972,-1.8610893755,-1.0322747441$ C, $-3.3579112114,-1.9949974411,-2.271381435$ $\mathrm{H},-4.1741380028,-2.6528548763,-2.5294812986$ C, $-2.7012807054,-1.0969176529,-3.0964697588$ C, $-0.9454204215,3.081635304,-0.2259684134$ $C_{,}-1.2001945873,3.9374516787,-1.3073644857$ $\mathrm{H},-1.3929485085,4.999504975,-1.2841155346$ C, $-1.1446488507,3.1115552226,-2.4177929853$ C, $1.1758958708,0.7736802617,1.4791005958$ $\mathrm{H}, 0.6741103144,1.46744308,2.1650591592$ C, $-1.5061737484,0.0010022593,1.7224542762$
$C,-3.1849012115,0.1017336143,3.6857919451$ $\mathrm{H},-4.1453431318,0.4942604207,3.3387381941$ $\mathrm{H},-2.806743114,0.7490785084,4.4826267315$ $\mathrm{B},-0.6917522484,0.5630617783,-2.8181611148$ $\mathrm{H},-0.8116252941,0.7955261061,-3.9923754665$ $\mathrm{H},-2.8424441654,-0.8555656777,-4.1400306148$ $\mathrm{H},-2.8943005573,-2.3791308243,-0.1004766822$ $\mathrm{H}, 1.5914849134,-0.1249764909,-4.4467464506$ $\mathrm{H}, 2.7591261116,-1.2965113697,-0.455754876$ $\mathrm{H},-1.2732959578,3.3239600453,-3.4692563753$ $\mathrm{H},-0.8855233486,3.3029787512,0.8306426666$ $\mathrm{H},-3.339399545,-0.9025369094,4.0920890228$ $\mathrm{H}, 1.7505203291,1.3782446464,0.7692246232$ C, $2.093578756,-0.1166338023,2.2558778417$ C, $1.6472642852,-0.8493247398,3.3694857566$ C, $2.5100138774,-1.6560287729,4.102920327$ C, $3.8559579223,-1.7609084717,3.7443743052$ C, $4.3225585855,-1.0374592973,2.6502061298$ C, $3.4529794568,-0.2240882293,1.9221012432$ $\mathrm{H}, 0.5989188504,-0.7886495286,3.652104143$ $\mathrm{H}, 2.1323146088,-2.2101925747,4.9591095377$ $\mathrm{H}, 4.5298444962,-2.3941265823,4.3149880113$ $\mathrm{H}, 5.3699578829,-1.0970017804,2.3638231459$ $\mathrm{H}, 3.8361275293,0.3553393822,1.0845736973$ 


\section{Cartesian coordinates for the Jones series $\mathrm{Rh}(\mathrm{H})(\mathrm{R})(\mathrm{Tp})\left(\mathrm{CNCH}_{3}\right)$ and enthalpies (au) at BP86}

\author{
Fragment $\operatorname{Rh}(\mathrm{Tp})(\mathrm{CNMe})(\mathrm{H})^{\bullet}: \mathrm{H}=-946.213599$
}

$\mathrm{Rh},-0.5508116692,-0.6643078881,-0.7523136617$ $\mathrm{H},-0.50096495,-2.2038670771,-1.0663506012$ $\mathrm{N},-1.0075023954,-1.1042373475,1.2632597091$ $\mathrm{N},-0.4271380385,-0.3591636406,2.2538370131$ $\mathrm{N}, 1.533804148,-0.5163644079,-0.0613053939$ $\mathrm{N}, 1.7549335326,0.1678791112,1.1004727361$ $\mathrm{N},-0.7297105907,1.4440168257,-0.1715435255$ $\mathrm{N},-0.1633846618,1.822908401,1.0136603851$ $\mathrm{N},-0.1427888209,-0.3076980602,-3.7735620955$ C, $-1.7848602445,-2.0286063789,1.867120946$ C, $-1.7178491709,-1.8807095476,3.2671021516$ $\mathrm{H},-2.230783224,-2.4690259527,4.0245219247$ C, $-0.842307399,-0.8117736752,3.4682964944$ $C, 2.7308715339,-0.9499620956,-0.5010878599$ C, $3.7519820473,-0.5436183479,0.3851896069$ $\mathrm{H}, 4.8195476695,-0.7388632107,0.3085038327$ C, $3.0874774199,0.1615525735,1.389361132$ C, $-1.2926530269,2.5481275448,-0.7040971611$ C, $-1.0942853759,3.6598879033,0.1420967133$ $\mathrm{H},-1.4297176431,4.6836031248,-0.0090666322$ C, $-0.3721057592,3.1530658168,1.2232369642$ $C_{,}-0.3209575478,-0.4390323043,-2.6007939709$ $C,-0.0033970232,-0.2277575824,-5.1793415042$ $\mathrm{H}, 0.7524089136,0.5301247993,-5.4495872965$ $\mathrm{H},-0.9627701611,0.0540734411,-5.6485369938$ B, $0.5693557778,0.7798785482,1.8980912172$ $\mathrm{H}, 0.9780859812,1.3046556282,2.9098183541$ $\mathrm{H}, 3.4569921721,0.6557912773,2.2861815174$ $\mathrm{H}, 2.7879682629,-1.5317234974,-1.41991554$ $\mathrm{H},-0.4821784484,-0.3400160835,4.3806594578$ $\mathrm{H},-2.3400359071,-2.7416663578,1.2601904653$ $\mathrm{H}, 0.0076133601,3.6338105785,2.1230457547$ $\mathrm{H},-1.8098916539,2.4806405478,-1.6603092521$ $\mathrm{H}, 0.3144869166,-1.2015046069,-5.5917291736$

$$
\mathrm{R}=\mathrm{Ph}, \mathrm{H}=-1177.788710
$$

$\mathrm{Rh}, 0.0542543828,-0.485359774,-0.4843126333$ $\mathrm{H},-0.2742219578,-1.9500880664,-0.8891325036$ $\mathrm{N},-0.7914053562,-0.8255662148,1.4144768157$ $\mathrm{N},-0.1406316608,-0.3681818292,2.5284132103$ $\mathrm{N}, 1.9407550873,-1.059546129,0.4589557695$ $\mathrm{N}, 2.2258654703,-0.5585190194,1.6973226814$ $\mathrm{N}, 0.5087982662,1.5449005477,0.2675710036$ $\mathrm{N}, 1.0088049862,1.6518896417,1.5351671349$ $\mathrm{N}, 1.2358420144,-0.1595303554,-3.2903930688$ C, $-1.9111556773,-1.4505587827,1.832233218$ $C_{,}-1.994254976,-1.4022986624,3.2383189277$ $\mathrm{H},-2.7757403583,-1.8140304868,3.8727762165$ C, $-0.8512972905,-0.707846008,3.6386339371$ C, $2.9638537867,-1.8693895066,0.1185447856$ C, $3.930507295,-1.8964454816,1.1458147467$ $\mathrm{H}, 4.864083117,-2.4542458432,1.1700606542$ C, $3.4193299401,-1.0509634423,2.1316376428$ C, $0.4457747722,2.7940692595,-0.2354650914$ C, $0.9101437186,3.7295783475,0.7138427285$ $\mathrm{H}, 0.9793372409,4.8101219863,0.6088153956$ C, $1.2571262884,2.9599745403,1.8252057991$ C, $0.7752715301,-0.2767780025,-2.2013387885$ C, $1.7212306396,0.0078911464,-4.6105918255$
$\mathrm{H}, 2.6863371254,0.5423892917,-4.5981337279$ $\mathrm{H}, 1.0009164719,0.5905739479,-5.2098626305$ $B, 1.2140991659,0.3833581945,2.4039866528$ $\mathrm{H}, 1.6237130153,0.6891657252,3.5013741995$ $\mathrm{H}, 3.8098379791,-0.7640240323,3.1063350747$ $\mathrm{H}, 2.9432396534,-2.3900990163,-0.837752336$ $\mathrm{H},-0.4885826232,-0.4279362416,4.6259170882$ $\mathrm{H},-2.5881234955,-1.882803077,1.0980129481$ $\mathrm{H}, 1.6586235785,3.2407046904,2.7973091971$ $\mathrm{H}, 0.0563903995,2.9491920103,-1.2407538616$ $\mathrm{H}, 1.8681503245,-0.9741475418,-5.0922101956$ $\mathrm{C},-1.7918797656,-0.0254527787,-1.2362561738$ C, $-2.3636961709,-0.6827529293,-2.353574573$ $C,-3.6502969942,-0.3581558754,-2.8203115228$ C, $-4.4074015696,0.6368541009,-2.1821113016$ $C,-3.8621289179,1.2988505726,-1.0706859794$ $C,-2.5749567124,0.9724968829,-0.6062193529$ $\mathrm{H},-1.800216031,-1.4717209504,-2.8642417197$ $\mathrm{H},-4.061929351,-0.8911162615,-3.6861038355$ $\mathrm{H},-5.4098516326,0.8909263731,-2.5438354408$ $\mathrm{H},-4.440235409,2.0759436135,-0.5559796447$ $\mathrm{H},-2.1768921327,1.5030259632,0.2648824705$

$$
\mathrm{R}=t \text {-Bu-vinyl, } \mathrm{H}=-1181.322748
$$

$\mathrm{Rh}, 0.2799079314,-0.393268929,-0.3235613062$ $\mathrm{H},-0.0845127321,-1.8441299458,-0.7484741265$ $\mathrm{N},-0.5784418327,-0.7428298514,1.5751577729$ $\mathrm{N}, 0.0763245812,-0.3144709308,2.6968525344$ $\mathrm{N}, 2.1627477985,-0.9906061161,0.6160895046$ $\mathrm{N}, 2.4450863244,-0.4988342159,1.8594755034$ $\mathrm{N}, 0.7303130455,1.6236322602,0.4583499785$ $\mathrm{N}, 1.2231029485,1.7146403424,1.7295581299$ $\mathrm{N}, 1.3898776415,-0.0937209308,-3.158528911$ $C_{,}-1.7141500044,-1.3465374573,1.9784208633$ $C,-1.8044358347,-1.3127637581,3.3849383004$ $\mathrm{H},-2.5977802615,-1.7149424302,4.0108902575$ C, $-0.648048787,-0.649520547,3.7998032733$ C, $3.1947417234,-1.7845177834,0.2658843168$ C, $4.1642053504,-1.8110664455,1.2907462523$ $\mathrm{H}, 5.1042045088,-2.3582804409,1.3075752972$ C, $3.6456676013,-0.981499366,2.285918228$ C, $0.6411402432,2.8812444129,-0.0182513199$ $C, 1.0809212804,3.8070089216,0.9523455481$ $\mathrm{H}, 1.1286521225,4.8906732059,0.8697686892$ C, $1.44061593,3.0215749465,2.0484363227$ C, $-1.5418509155,0.165206057,-1.0159798071$ $\mathrm{H},-1.8545999935,1.1364452093,-0.5950320684$ C, $-2.3879520246,-0.4852733865,-1.8430117348$ $\mathrm{H},-2.0828089024,-1.4559815476,-2.2665084728$ C, $0.9643532966,-0.1981697293,-2.0536717006$ C, $1.8369988485,0.0060490267,-4.4984222982$ $\mathrm{H}, 2.7999827007,0.542933954,-4.5425041445$ $\mathrm{H}, 1.0983220598,0.554822231,-5.1073156077$ $\mathrm{B}, 1.4333057872,0.4324846957,2.5798560747$ $\mathrm{H}, 1.845924604,0.7247396738,3.6799886838$ $\mathrm{H}, 4.0348383585,-0.7003176103,3.2628319778$ $\mathrm{H}, 3.1782885609,-2.2947611739,-0.6961381169$ $\mathrm{H},-0.2849142428,-0.3889300022,4.7922425449$ $\mathrm{H},-2.3934103624,-1.7527484126,1.2314610435$ $\mathrm{H}, 1.8312406434,3.2907288399,3.0282195978$ $\mathrm{H}, 0.2644580996,3.0490762051,-1.0266292453$ 
$\mathrm{H}, 1.9737945014,-0.9993678747,-4.9329897361$ C, $-3.7856002305,-0.0452032208,-2.2873192763$ C, $-4.8060863212,-1.1207531861,-1.8306340705$ $\mathrm{H},-4.5335204561,-2.1184870047,-2.2186283145$ $\mathrm{H},-4.8432956675,-1.1863324081,-0.7294143878$ $\mathrm{H},-5.8227863832,-0.8830652105,-2.1946712733$ C, $-4.1971346527,1.3229094852,-1.7089057128$ $\mathrm{H},-5.2107640296,1.5961546491,-2.0524146529$ $\mathrm{H},-4.2064872448,1.3084981516,-0.6053942469$ $\mathrm{H},-3.5048932283,2.1201707526,-2.0311594629$ $C,-3.8036624177,0.0296415753,-3.8365717613$ $\mathrm{H},-3.1066100801,0.8043745075,-4.2005664372$ $\mathrm{H},-3.5020674985,-0.9344886445,-4.283925107$ $\mathrm{H},-4.8150529869,0.2732424791,-4.2112684155$

\section{$\mathrm{R}=\mathrm{Me}, \mathrm{H}=-986.107996$}

$\mathrm{Rh},-0.5316808366,-0.4162332051,-0.7999227101$ $\mathrm{H},-0.8920418437,-1.8738067836,-1.21648815$ $\mathrm{N},-1.3691437855,-0.764420946,1.1064982964$ $\mathrm{N},-0.7229317897,-0.2932996634,2.216316086$ $\mathrm{N}, 1.3564591442,-0.9867638073,0.1500787056$ $\mathrm{N}, 1.6423178421,-0.4591252818,1.3776617357$ $\mathrm{N},-0.1044036852,1.6182608151,-0.0556433351$ $\mathrm{N}, 0.3919258041,1.7383051972,1.2116336066$ $\mathrm{N}, 0.5675250331,-0.1230346543,-3.6360122471$ $C_{,}-2.4658551539,-1.4230335788,1.5348075992$ C, $-2.5402293957,-1.3787412397,2.9417031257$ $\mathrm{H},-3.3034753265,-1.814699864,3.5822260151$ C, $-1.4133266448,-0.6537604715,3.3327519623$ C, $2.3912784532,-1.7842016961,-0.1831243073$ C, $3.3662124274,-1.7770217807,0.8373416407$ $\mathrm{H}, 4.3093187267,-2.3185220455,0.8646183549$ C, $2.8476132392,-0.9235081663,1.8118535283$ C, $-0.2066229341,2.8658721546,-0.5545574064$ C, $0.2276365637,3.814017665,0.3968907056$ $\mathrm{H}, 0.2646614295,4.8963339049,0.2936451899$ C, $0.5984373725,3.0529300681,1.5060763975$ C, $-2.3990669743,0.1213131529,-1.5517228528$ $\mathrm{H},-2.37586278,1.1737234446,-1.8884229959$ C, $0.149049467,-0.2293226074,-2.5266279406$ C, $1.0814279159,-0.0520243354,-4.9533024025$ $\mathrm{H}, 2.1072880478,0.3551260279,-4.9444186389$ $\mathrm{H}, 0.4497196152,0.6033685425,-5.5773752782$ $\mathrm{B}, 0.6214590303,0.4754980593,2.0815283839$ $\mathrm{H}, 1.0304271971,0.791743254,3.1764721166$ $\mathrm{H}, 3.2397201259,-0.613365858,2.7787947524$ $\mathrm{H}, 2.3719764501,-2.3221184378,-1.1298967071$ $\mathrm{H},-1.048826644,-0.3665053573,4.3172262066$ $\mathrm{H},-3.1305113038,-1.8895434338,0.8099651729$ $\mathrm{H}, 0.9899910374,3.3437043783,2.479293612$ $\mathrm{H},-0.5896134175,3.0138366493,-1.5635377253$ $\mathrm{H}, 1.1045244366,-1.0559637369,-5.4120535002$ $\mathrm{H},-3.134311875,0.0357588865,-0.7336615966$ $\mathrm{H},-2.7262073385,-0.510632243,-2.3937770286$

\section{$\mathrm{R}=\mathrm{Pe}, \mathrm{H}=-1143.240332$}

$\mathrm{Rh}, 0.2566003594,-0.2308201545,-0.3319351858$ $\mathrm{H}, 0.3523119706,-1.7484982926,-0.6687864668$ $\mathrm{N},-0.2436154201,-0.7168735102,1.6624598835$ $\mathrm{N}, 0.3326760995,-0.0163004997,2.6861465308$ $\mathrm{N}, 2.3209106221,-0.1355693913,0.4218500133$ $\mathrm{N}, 2.5406708937,0.5170790713,1.6019878257$ $\mathrm{N}, 0.09204634,1.8688691399,0.323423972$ $\mathrm{N}, 0.6502383029,2.2029064311,1.5251174488$
$\mathrm{N}, 0.9626797014,0.2306969741,-3.2702205489$ C, $-1.0494491652,-1.6442220963,2.219525004$ C, $-1.0013480685,-1.548187521,3.6249784019$ $\mathrm{H},-1.5357108357,-2.1544415946,4.3528385596$ C, $-0.1108803265,-0.5031025012,3.8774668489$ C, $3.5181456118,-0.5856872,-0.0040687423$ C, $4.5331416968,-0.2225630672,0.9070413953$ $\mathrm{H}, 5.5971404409,-0.4403828872,0.844338333$ C, $3.8664344771,0.4751836218,1.914875876$ C, $-0.4423958724,2.9965166535,-0.1857380974$ $C,-0.2317923082,4.081502669,0.692353693$ $\mathrm{H},-0.5439337373,5.1157202526,0.5643510755$ C, $0.4659980044,3.531176818,1.7681108324$ C, $-1.7687227546,-0.3091436953,-0.8600626397$ $\mathrm{H},-2.000436127,0.6084639097,-1.4372971275$ C, $0.6872307845,0.0590099686,-2.1243882922$ C, $1.3349586068,0.3759329452,-4.6285541852$ $\mathrm{H}, 2.0235552672,1.2299933769,-4.7492542249$ $\mathrm{H}, 0.4433497291,0.5534656188,-5.2543214791$ $\mathrm{B}, 1.349192014,1.1222644587,2.3911859195$ $\mathrm{H}, 1.7408749377,1.6087117373,3.4286606941$ $\mathrm{H}, 4.2304187256,0.9411719514,2.8288631595$ $\mathrm{H}, 3.5809124995,-1.1502254475,-0.9333870831$ $\mathrm{H}, 0.2407673073,-0.0674958142,4.8108215312$ $\mathrm{H},-1.6116934986,-2.3219369059,1.5793959441$ $\mathrm{H}, 0.8432086691,3.9794419534,2.6855364384$ $\mathrm{H},-0.9512813831,2.9649120474,-1.1483838131$ $\mathrm{H}, 1.8397793555,-0.5372736238,-4.9894650753$ $\mathrm{H},-2.3078887439,-0.2223813803,0.1027610016$ C, $-2.2694805748,-1.5381352833,-1.6316280423$ $\mathrm{H},-1.721437737,-1.634143093,-2.5898216336$ $\mathrm{H},-2.03581887,-2.4605397493,-1.0637222005$ $\mathrm{C},-3.7852316205,-1.5000385457,-1.9203596641$ $\mathrm{H},-4.0246875239,-0.5889238223,-2.5057721047$ $\mathrm{H},-4.3361943358,-1.3967246013,-0.9635800568$ C, $-4.3048721991,-2.7386399812,-2.6727454951$ $\mathrm{H},-3.7582980745,-2.8377865706,-3.6312204771$ $\mathrm{H},-4.0577042657,-3.6470220897,-2.0889582724$ C, $-5.816769594,-2.6992539523,-2.9445812993$ $\mathrm{H},-6.0908316196,-1.8199230415,-3.5546748957$ $\mathrm{H},-6.1585317916,-3.5994984257,-3.4841745468$ $\mathrm{H},-6.3898925657,-2.6368493497,-2.002302955$

\section{$\mathrm{R}=c-\mathrm{Pe}, \mathrm{H}=-1142.048311$}

$\mathrm{Rh},-0.3672449905,0.1749237318,-0.4782371511$ $\mathrm{H},-0.7773268503,0.1291856834,-1.9768856218$ $\mathrm{N}, 0.8475960367,-1.5288922756,-0.7813414542$ $\mathrm{N}, 2.0955882489,-1.5722960009,-0.2235116397$ $\mathrm{N}, 1.4828088493,1.3033579163,-0.9007959363$ $\mathrm{N}, 2.6463976899,0.8792243088,-0.3252230654$ $\mathrm{N}, 0.3804980491,0.1578608994,1.6196107301$ $\mathrm{N}, 1.7101294609,-0.1040843493,1.804382157$ $\mathrm{N},-2.1361715716,2.6610207122,-0.2886993969$ C, $0.6882184815,-2.6688338803,-1.4858673457$ C, $1.8437885109,-3.4690596525,-1.3838694134$ $\mathrm{H}, 2.0222615976,-4.4417177971,-1.8365299034$ C, $2.7123074149,-2.7335286062,-0.5757390612$ C, $1.7900706735,2.3761630469,-1.6574446109$ C, $3.1704229926,2.6584409343,-1.5735790007$ $\mathrm{H}, 3.7209929807,3.4559835917,-2.067850937$ C, $3.6769791059,1.679891028,-0.7171156577$ C, $-0.1290110161,0.4395902798,2.8363910933$ C, $0.873632165,0.3562111304,3.8259725869$ $\mathrm{H}, 0.7717090135,0.520067492,4.8965816915$ 
C, $2.0281781224,0.0087994616,3.1248226976$ $C_{,}-1.460105636,1.6823087618,-0.3471123892$ $C_{,}-2.969819301,3.8029952495,-0.2168651663$ $\mathrm{H},-2.4688261584,4.6093539506,0.3460138366$ $\mathrm{H},-3.917824533,3.5560159114,0.2920650035$ B, 2.6417721021, $-0.371063222,0.5956426324$ $\mathrm{H}, 3.7648540212,-0.6101723194,0.9803190964$ $\mathrm{H}, 4.687330294,1.4904183392,-0.3588949537$ $\mathrm{H}, 1.0061819847,2.8777393778,-2.2233186228$ $\mathrm{H}, 3.7203946476,-2.9449432921,-0.2241829322$ $\mathrm{H},-0.241761709,-2.8441733549,-2.0238682429$ $\mathrm{H}, 3.0486252749,-0.1669352223,3.4605967625$ $\mathrm{H},-1.1864509639,0.6781729265,2.935073802$ $\mathrm{H},-3.2026421204,4.1738527262,-1.2302244796$ $C,-2.0301137165,-1.0736578539,-0.1744622518$ $C_{,}-2.8401119428,-0.9208480224,1.1317721745$ C, $-4.1079335775,-1.7995278253,0.9138198854$ C, $-4.287547823,-1.9103293407,-0.6371052497$ C, $-3.1426310359,-1.0546564047,-1.2459606327$ $\mathrm{H},-1.577450215,-2.086478729,-0.1655096805$ $\mathrm{H},-3.1404297747,0.136706802,1.2712172966$ $\mathrm{H},-2.2735876402,-1.2218557624,2.0282244878$ $\mathrm{H},-3.9596728847,-2.8002508231,1.3562420107$ $\mathrm{H},-4.9962496326,-1.3671813152,1.4071339266$ $\mathrm{H},-4.1894446208,-2.9627043423,-0.9580491745$ $\mathrm{H},-5.2846993743,-1.5720598437,-0.9695347217$ $\mathrm{H},-2.804588334,-1.4269054509,-2.2290395855$ $\mathrm{H},-3.490098794,-0.0154586809,-1.4040161312$

\section{$\mathrm{R}=\mathrm{Cy}, \mathrm{H}=-1181.338951$}

$\mathrm{Rh},-0.1617332533,0.2386387263,-0.498043265$ $\mathrm{H},-0.5482949686,0.1488143551,-1.9982224395$ $\mathrm{N}, 0.6898276154,-1.6952574825,-0.6254826143$ $\mathrm{N}, 1.9032325342,-1.9363339707,-0.0423427051$ $\mathrm{N}, 1.8793766906,0.9346198148,-0.9903133611$ $\mathrm{N}, 2.9391689061,0.330608367,-0.3770440744$ $\mathrm{N}, 0.5972256714,0.2887233002,1.6071539941$ $\mathrm{N}, 1.8465509356,-0.2235801261,1.8270502714$ $\mathrm{N},-1.4141239967,3.0282698079,-0.5490699699$ C, $0.3036909256,-2.8418522541,-1.2238086761$ C, $1.2729885139,-3.8452251257,-1.0250636987$ $\mathrm{H}, 1.2507762955,-4.8725399585,-1.3813796531$ C, $2.2721423531,-3.2263003598,-0.2723374786$ C, $2.3898765332,1.8408275298,-1.8482700884$ C, $3.8002306485,1.8313505979,-1.7926398415$ $\mathrm{H}, 4.4960810585,2.4441732936,-2.3616589005$ C, $4.1071180104,0.8537900945,-0.8450462225$ C, $0.1961148705,0.8339220517,2.7745559769$ C, $1.1857340576,0.6701353964,3.7669749628$ $\mathrm{H}, 1.1509047473,0.9921930943,4.8054856531$ C, $2.2195683013,-0.0058864457,3.1198522315$ $C_{,}-0.9437037048,1.9345012003,-0.5143459872$ C, $-2.0045476311,4.3140312289,-0.5972217651$ $\mathrm{H},-1.3651566612,5.0499572021,-0.0798313386$ $\mathrm{H},-2.993685679,4.3021938842,-0.1071608638$ $\mathrm{B}, 2.6891147317,-0.7971724136,0.6598492725$ $\mathrm{H}, 3.7435270074,-1.2213182141,1.0782663421$ $\mathrm{H}, 5.0620540789,0.4905543926,-0.4691886927$ $\mathrm{H}, 1.7175287589,2.4403697002,-2.460500155$ $\mathrm{H}, 3.2170665954,-3.6017120974,0.1158633589$ $\mathrm{H},-0.6419836969,-2.8782736184,-1.7616530729$ $\mathrm{H}, 3.1893117802,-0.3489636535,3.4759628751$ $\mathrm{H},-0.7822238732,1.3066167147,2.8409042333$ $\mathrm{H},-2.1362599802,4.6379106866,-1.6443545391$
$C,-4.0405734363,-0.9945285936,1.4478907303$ $C_{,}-2.7144268548,-0.2361546679,1.1935900129$ $C,-2.061361148,-0.6386555052,-0.1417919348$ $C,-3.0673564926,-0.4646140278,-1.2996965703$ $C,-4.3922072374,-1.2227161816,-1.048997315$ C, $-5.0333641691,-0.807615453,0.2870889651$ $\mathrm{H},-2.9267923795,0.8537011512,1.1936539157$ $\mathrm{H},-2.0227353333,-0.4280664632,2.0310872886$ $\mathrm{H},-3.8198570568,-2.0747643607,1.5639585822$ $\mathrm{H},-4.4908520226,-0.6627710128,2.4031874434$ $\mathrm{H},-3.2943288141,0.6131386401,-1.4323953348$ $\mathrm{H},-2.6214873992,-0.8000336476,-2.2535923856$ $\mathrm{H},-5.0944110635,-1.052747265,-1.8875126143$ $\mathrm{H},-4.1878874677,-2.3125487766,-1.0267631679$ $\mathrm{H},-5.3315556782,0.2594895514,0.2309698803$ $\mathrm{H},-5.9605863067,-1.3821492924,0.4714684847$ $\mathrm{H},-1.8050789373,-1.7155098066,-0.0679549294$

\section{$\mathrm{R}=$ Methallyl, $\mathrm{H}=-1102.754457$}

$\mathrm{Rh}, 0.6077225932,-0.1071308868,-0.2051419466$ $\mathrm{H}, 1.3521612123,0.2221215835,-1.5325542901$ $\mathrm{N},-0.5693617933,1.6297509283,-0.4608794499$ $\mathrm{N},-1.9145625239,1.5628336533,-0.2142623304$ $\mathrm{N},-1.0300018307,-1.1231802245,-1.2392054818$ $\mathrm{N},-2.3077937622,-0.8367614408,-0.8500487651$ $\mathrm{N},-0.559181128,-0.4263138583,1.6379480953$ $\mathrm{N},-1.9084117536,-0.2213345058,1.5750473492$ $\mathrm{N}, 2.3848823936,-2.5920969364,-0.0543110355$ C, $-0.3056867142,2.8727354104,-0.9115086047$ C, $-1.4930730797,3.6319026875,-0.9544307454$ $\mathrm{H},-1.6095139332,4.6669468361,-1.2677417293$ C, $-2.488814612,2.7618743972,-0.5074973077$ $C,-1.1160381077,-2.0391073808,-2.2248730206$ C, $-2.4665526539,-2.355991017,-2.4836574936$ $\mathrm{H},-2.86031542,-3.0539490973,-3.2193399436$ C, $-3.1900046548,-1.5666214407,-1.5886123052$ $C,-0.2783670086,-0.796203315,2.9033633236$ $C,-1.4576795321,-0.8343132085,3.6779417575$ $\mathrm{H},-1.5588515065,-1.093925247,4.7295019957$ C, $-2.4702079023,-0.4607070649,2.7932269571$ C, $2.0449862,1.0224898377,0.8707607073$ $\mathrm{H}, 2.5462651118,0.3155760343,1.559271817$ C, $1.6981340111,-1.6207408212,-0.1067504451$ C, $3.185499164,-3.7602728966,-0.0674486098$ $\mathrm{H}, 2.5560566954,-4.6580890847,0.0585057612$ $\mathrm{H}, 3.9218726394,-3.7305212635,0.7539411265$ $B,-2.568422179,0.2327457372,0.2465843218$ $\mathrm{H},-3.7594260511,0.3843098744,0.4037758237$ $\mathrm{H},-4.2610396007,-1.4645298767,-1.4228940041$ $\mathrm{H},-0.206738215,-2.4094316492,-2.696106391$ $\mathrm{H},-3.5600382306,2.9016497573,-0.3742615663$ $\mathrm{H}, 0.7211488757,3.1416018666,-1.1583218649$ $\mathrm{H},-3.5431352473,-0.344976596,2.9358805808$ $\mathrm{H}, 0.7518525389,-1.0077745488,3.1874455529$ $\mathrm{H}, 3.730899296,-3.8423524066,-1.023776615$ $\mathrm{H}, 1.42893963,1.7120988423,1.4727242719$ C, $3.070480767,1.7738994475,0.0801602234$ C, $3.0904971527,3.1286721022,0.0287622876$ $\mathrm{H}, 3.8549727228,3.6718657017,-0.5384397798$ $\mathrm{H}, 2.3574943698,3.7329436096,0.5745972861$ C, $4.1334696506,0.9654447896,-0.6351015152$ $\mathrm{H}, 4.6708990442,0.3100282249,0.0766466261$ $\mathrm{H}, 3.6764784101,0.3046093586,-1.3931209148$ $\mathrm{H}, 4.8757685634,1.6103068258,-1.1332976806$ 


\section{$\mathrm{R}=$ Mesityl, $\mathrm{H}=-1295.647805$}

$\mathrm{Rh},-0.5961230476,0.0165736796,0.0592201982$ $\mathrm{H},-0.224989239,-1.3746922016,0.6447551997$ $\mathrm{N}, 0.8094035799,-0.2854146146,-1.4919436176$ $\mathrm{N}, 0.4714147358,0.0703606856,-2.770464454$ $\mathrm{N},-2.0310411711,-0.8236642446,-1.3616722122$ $\mathrm{N},-2.0003737014,-0.3652884104,-2.6484269639$ $\mathrm{N},-1.0089865362,1.9592207767,-0.9079332188$ $\mathrm{N},-1.1101986866,1.9771556365,-2.2700112529$ $\mathrm{N},-2.5848067057,0.2245680803,2.3740736771$ C, $2.03162655,-0.8532108124,-1.5457265274$ C, $2.5027010161,-0.8622336206,-2.8745351827$ $\mathrm{H}, 3.4514853958,-1.2473719733,-3.2413203238$ C, $1.4806434187,-0.2703685327,-3.6181900615$ C, $-3.0224032987,-1.7348136503,-1.2929325756$ C, $-3.6483020388,-1.8744188951,-2.5499664099$ $\mathrm{H},-4.4743806233,-2.5303782359,-2.8160558022$ C, $-2.9648519259,-0.9874303808,-3.3828965242$ C, $-1.1961959033,3.2256356394,-0.4856467801$ $C,-1.4228096453,4.0825001606,-1.584292651$ $\mathrm{H},-1.6050575888,5.1549071595,-1.5696218195$ C, $-1.3581621333,3.246366798,-2.69975432$ C, $0.9000469232,0.9088538884,1.2645998704$ $\mathrm{H}, 0.3799119795,1.5802865747,1.9716179907$ C, $-1.8098123335,0.1487017306,1.4730075075$ $C,-3.5359161906,0.2739078914,3.4223306502$ $\mathrm{H},-4.5083887336,0.6312770148,3.04183017$
$\mathrm{H},-3.1961815206,0.9606602859,4.2166551383$ $\mathrm{B},-0.9266556869,0.6696845299,-3.0834686583$ $\mathrm{H},-1.0263802009,0.8996694933,-4.2681580166$ $\mathrm{H},-3.0865904602,-0.754175803,-4.4392229131$ $\mathrm{H},-3.2219645246,-2.2425210583,-0.3503295593$ $\mathrm{H}, 1.3914744949,-0.0657768554,-4.6835505237$ $\mathrm{H}, 2.4964691772,-1.2087046833,-0.6271222525$ $\mathrm{H},-1.467328119,3.4553005234,-3.7625023151$ $\mathrm{H},-1.1515634903,3.451264796,0.5792392477$ $\mathrm{H},-3.6762475272,-0.7286773682,3.8626533335$ $\mathrm{H}, 1.4582284116,1.5393224737,0.5510055944$ C, $1.8402360167,0.0083805036,2.0139102362$ C, $1.4063569615,-0.7664743471,3.1169985431$ C, $2.2831348737,-1.5910060497,3.8368407246$ C, $3.6388893828,-1.6482154461,3.4429044998$ C, $4.1143685259,-0.8877870658,2.3624270625$ C, $3.2044407244,-0.0672135787,1.6611859035$ $\mathrm{H}, 0.3475247762,-0.7320992395,3.4018911443$ $\mathrm{H}, 4.3339244003,-2.2960575294,3.9926438391$ $\mathrm{H}, 3.5695091214,0.5434693067,0.8251345869$ C, $5.5787610416,-0.9181955239,1.9727762057$ $\mathrm{H}, 6.1104035379,-0.0193407631,2.3371561073$ $\mathrm{H}, 6.092261264,-1.7974049985,2.3963403302$ $\mathrm{H}, 5.7083761237,-0.9436664538,0.8768426284$ C, $1.7977682201,-2.401962633,5.0216371233$ $\mathrm{H}, 2.2187838621,-2.0232519544,5.9716333748$ $\mathrm{H}, 0.6990719565,-2.3699257722,5.1090319759$ $\mathrm{H}, 2.0994613856,-3.4610127898,4.9384697648$ 


\section{Cartesian coordinates for the Wolczanski series Ti(OSit-Bu $)_{2}(\mathrm{NHSi} t-\mathrm{Bu})(\mathrm{R})$ and en- thalpies (au) at B3PW91}

\author{
Fragment Ti(OSit-Bu $)_{2}(\mathrm{NHSi}-\mathrm{Bu})^{\bullet}, \mathrm{H}=-634.871513$
}

Ti, $0.0595072246,-0.3027986689,-0.0185683142$ $\mathrm{Si},-3.0118032441,-1.8074225987,0.0178755674$ Si, $3.42845659,-1.211479067,0.0125901418$ $\mathrm{N},-1.2430905888,-1.691347748,0.0514469917$ $0,1.7778623269,-0.8462607661,-0.0034538125$ C, $3.6117293052,-3.0843488973,0.1653689854$ $C, 4.214630434,-0.3452748034,1.4942479934$ C, $4.1868517275,-0.5955848734,-1.6024172794$ $0,-0.446870455,1.4322615259,-0.0387314337$ Si, $-0.4439959764,3.1229203953,-0.0212600239$ $C_{,}-1.6910951714,3.7279864647,-1.3020795209$ C, $-0.9324593528,3.6916460734,1.711267319$ C, $1.2915892207,3.7306691965,-0.4540360509$ $C,-3.6661354804,-2.3035828824,1.726121567$ $\mathrm{H},-3.2524863548,-3.267419727,2.0417737432$ $\mathrm{H},-3.3870937515,-1.5578587852,2.4774983431$ $\mathrm{H},-4.7581916815,-2.3929017253,1.7237180428$ $C,-3.707855685,-0.1230164525,-0.4757249553$ $\mathrm{H},-3.3913130806,0.6561665792,0.2238810976$ $\mathrm{H},-3.3662597871,0.1622259918,-1.4756263201$ $\mathrm{H},-4.8029102082,-0.1474840391,-0.4850079924$ C, $-3.5267566122,-3.1212677758,-1.2482733426$ $\mathrm{H},-4.6169435615,-3.2211480753,-1.2920693002$ $\mathrm{H},-3.1695730729,-2.8577817743,-2.248909271$ $\mathrm{H},-3.1149084705,-4.1036362427,-0.9920521095$ $\mathrm{H}, 5.2877893571,-0.5579071579,1.5504575116$ $\mathrm{H}, 4.0888451467,0.7397703142,1.4227977098$ $\mathrm{H}, 3.7545057658,-0.6791826543,2.4297057924$ $\mathrm{H}, 3.1322234703,-3.59298587,-0.6771118041$ $\mathrm{H}, 4.667161931,-3.3773735356,0.1803194119$ $\mathrm{H}, 3.1486285468,-3.4459101833,1.0891112594$ $\mathrm{H}, 3.703064606,-1.0669290432,-2.4637955605$ $\mathrm{H}, 4.0692436358,0.4884270037,-1.6987561833$ $\mathrm{H}, 5.257126311,-0.824757518,-1.6479962563$ $\mathrm{H}, 1.336076682,4.8252713267,-0.4616525263$ $\mathrm{H}, 1.5914098383,3.3740310709,-1.4447292332$ $\mathrm{H}, 2.0247652095,3.3684012044,0.2738081387$ $\mathrm{H},-1.417967237,3.3817310337,-2.3039832556$ $\mathrm{H},-2.6945208799,3.3539042747,-1.0771148183$ $\mathrm{H},-1.7332539977,4.8224411409,-1.3224219728$ $\mathrm{H},-1.9266177411,3.3190512567,1.9775504655$ $\mathrm{H},-0.9524423744,4.7850082195,1.7753795483$ $\mathrm{H},-0.221675004,3.322082969,2.4573211923$ $\mathrm{H},-0.8103365777,-2.6118556935,0.1380323978$

\section{$\mathrm{R}=\mathrm{Ph}, \mathrm{H}=-866.353140$}

Ti, $0.0980678572,-0.0818548838,-0.19052965$ Si, $-2.3063493775,-2.2756433465,-1.0759617829$ Si, $3.3034807295,-1.2403578165,0.3596019476$ $\mathrm{N},-0.7288360993,-1.4569007704,-1.1731232754$ $0,1.8063285831,-0.471030203,0.1603089888$ C, $3.6400473257,-2.2814140389,-1.178452807$ C, $3.2059599469,-2.3271319253,1.8985389892$ C, $4.6132943462,0.101939164,0.5573084378$ $0,-0.9008519357,0.3635330728,1.2229617446$ Si, $-1.2400888149,1.2262415811,2.6474628819$ $C_{,}-2.7705882318,2.2797778274,2.3304068455$ C, $-1.5534459823,-0.0352198448,4.0158119164$ C, $0.2480612109,2.3104975749,3.0577678658$
$C,-2.3146731626,-3.5143778462,0.3564718249$ $\mathrm{H},-1.5409399805,-4.2770353888,0.2193241991$ $\mathrm{H},-2.1190832499,-3.0013608134,1.3036397429$ $\mathrm{H},-3.2818175238,-4.023085991,0.4373888035$ C, $-3.6500440179,-0.9791720427,-0.8043066447$ $\mathrm{H},-3.4080814624,-0.3572050141,0.0628337315$ $\mathrm{H},-3.7443103106,-0.3240941555,-1.6759643522$ $\mathrm{H},-4.621203377,-1.453676524,-0.6268914833$ C, $-2.580568415,-3.1935522923,-2.7108804285$ $\mathrm{H},-3.540490173,-3.7214104333,-2.7018566113$ $\mathrm{H},-2.5853430991,-2.4983135053,-3.5565272352$ $\mathrm{H},-1.7961782025,-3.9379686654,-2.886889081$ $\mathrm{H}, 4.1531980031,-2.8502351301,2.0694773282$ $\mathrm{H}, 2.9858016293,-1.7257588477,2.7861685806$ $\mathrm{H}, 2.4171491094,-3.0787149526,1.7924292264$ $\mathrm{H}, 3.619736633,-1.6575541907,-2.0778336906$ $\mathrm{H}, 4.6234710912,-2.7605404695,-1.1203231071$ $\mathrm{H}, 2.8888668307,-3.0701347519,-1.2904571751$ $\mathrm{H}, 4.6377682109,0.7485723351,-0.325324628$ $\mathrm{H}, 4.403098318,0.725917631,1.4316544947$ $\mathrm{H}, 5.6087047991,-0.3371709789,0.6851571864$ $\mathrm{H}, 0.0763774649,2.8801450837,3.9776167421$ $\mathrm{H}, 0.4382189363,3.0215827959,2.2476031551$ $\mathrm{H}, 1.1471563651,1.7020365145,3.1978460038$ $\mathrm{H},-2.5957291364,2.9669690572,1.4967618723$ $\mathrm{H},-3.6324861386,1.6533625415,2.0804410818$ $\mathrm{H},-3.0278648663,2.8730435674,3.2147557722$ $\mathrm{H},-2.4008379002,-0.6804792763,3.7640040033$ $\mathrm{H},-1.7783484374,0.4642118509,4.9645598382$ $\mathrm{H},-0.674616733,-0.6702376482,4.1662988146$ $\mathrm{H},-0.0870854973,-1.9196245821,-1.8164305709$ C, $0.1820604854,1.586209557,-1.4467781007$ C, $1.172676351,2.5614842444,-1.2243426972$ C, $1.2297281695,3.7325436684,-1.9823918435$ C, $0.2919914802,3.9554170358,-2.99012749$ $C_{,}-0.6969943143,3.0032424091,-3.2360300588$ C, $-0.7473374117,1.8354751527,-2.4731070003$ $\mathrm{H}, 1.9227059998,2.4048037909,-0.4505196649$ $\mathrm{H}, 2.0073696865,4.4686129026,-1.7905756551$ $\mathrm{H}, 0.3348343287,4.8647833391,-3.5844406557$ $\mathrm{H},-1.4270745233,3.1697272457,-4.0250000049$ $\mathrm{H},-1.5220223355,1.1022448261,-2.6863528075$

$$
\mathrm{R}=\text { Vinyl, } \mathrm{H}=-712.803025
$$

$\mathrm{Ti},-0.0413969296,-0.2289249271,0.6180982891$ $\mathrm{Si}, 2.5553655883,-2.1595729565,-0.3998173787$ $\mathrm{Si},-3.2971396723,-0.6264054394,-0.4705665197$ $\mathrm{N}, 0.9025515226,-1.784943618,0.1342110233$ $0,-1.7802715184,-0.4231537055,0.258631738$ $C,-3.8527142749,-2.4023918471,-0.1578048926$ $C_{,}-3.1128676428,-0.2956541253,-2.3193130997$ $C,-4.4949853744,0.5999224337,0.315976363$ $0,0.7494645272,1.248433295,-0.006152515$ $\mathrm{Si}, 0.896234581,2.9083755881,-0.3311885514$ C, $2.2988354595,3.582588064,0.7335950075$ C, $1.2898597377,3.1002052091,-2.1664776141$ C, $-0.7359080031,3.7507884264,0.1015048862$ C, $2.6170488727,-2.2893035177,-2.2888040378$ $\mathrm{H}, 1.9388851739,-3.0697422801,-2.6501214144$ $\mathrm{H}, 2.3151409057,-1.3429431247,-2.7490312895$ $\mathrm{H}, 3.6262311921,-2.5323914729,-2.6398219722$ 
C, $3.702280867,-0.7831567375,0.1924660724$ $\mathrm{H}, 3.3479947889,0.1907171516,-0.1574682074$ $\mathrm{H}, 3.7448828552,-0.7560922281,1.2859389868$ $\mathrm{H}, 4.7195919068,-0.9347339825,-0.1840003202$ C, $3.0580713903,-3.8199994552,0.3612042469$ $\mathrm{H}, 4.0737462177,-4.0973181303,0.0584190346$ $\mathrm{H}, 3.030635023,-3.7707450519,1.4543475323$ $\mathrm{H}, 2.3870451508,-4.6250026266,0.0417240516$ $\mathrm{H},-4.0646177898,-0.4430552282,-2.8413826152$ $\mathrm{H},-2.7842885271,0.7325565107,-2.500841721$ $\mathrm{H},-2.3746613066,-0.9711733648,-2.76328751$ $\mathrm{H},-3.8927944885,-2.612435962,0.9156455821$ $\mathrm{H},-4.8491956365,-2.5822731886,-0.5757893879$ $\mathrm{H},-3.1611718548,-3.1153634404,-0.6181848731$ $\mathrm{H},-4.5889115367,0.4207906938,1.3915431806$ $\mathrm{H},-4.1501503679,1.6287470399,0.173509968$ $\mathrm{H},-5.4918731833,0.5117227907,-0.1297347101$ $\mathrm{H},-0.6797634537,4.8311877153,-0.0712997408$ $\mathrm{H},-0.9848304937,3.5873233335,1.1548585373$ $\mathrm{H},-1.5540756417,3.3533216533,-0.5073067513$ $\mathrm{H}, 2.0878227745,3.4201728157,1.7951558291$ $\mathrm{H}, 3.2456298895,3.087798297,0.4960051547$ $\mathrm{H}, 2.4292923399,4.6584589062,0.573376773$ $\mathrm{H}, 2.2204165331,2.5832734946,-2.4212089115$ $\mathrm{H}, 1.406245236,4.1564561275,-2.4327475089$ $\mathrm{H}, 0.4893969693,2.6816047981,-2.784632382$ $\mathrm{H}, 0.3152327985,-2.6169145827,0.1919208674$ C, $0.1052769253,-0.1282051628,2.6839898802$ C, $-0.9446340328,-0.122487121,3.5200772889$ $\mathrm{H}, 1.1011083472,-0.0673292445,3.1385941275$ $\mathrm{H},-0.8381531889,-0.0600365981,4.6058973575$ $\mathrm{H},-1.9695134169,-0.1852615079,3.1559787161$

\section{$\mathrm{R}=\mathrm{Me}, \mathrm{H}=-674.752427$}

$$
\begin{array}{rrr}
-0.02409 & -0.29985 & -0.63561 \\
2.93426 & -1.64065 & 0.28476 \\
0.33988 & 3.00025 & 0.18445 \\
1.18787 & -1.61281 & -0.03433 \\
0.50143 & 1.36485 & -0.23056 \\
1.88524 & 3.90023 & -0.41547 \\
0.17723 & 3.1212 & \multicolumn{1}{c}{2.061} \\
-1.20089 & 3.68527 & -0.66391 \\
-1.6871 & -0.75036 & -0.17286 \\
-3.26073 & -1.17372 & 0.28903 \\
-3.49103 & -3.0148 & -0.05571 \\
-3.44828 & -0.80867 & 2.13085 \\
-4.46958 & -0.14578 & -0.73038 \\
3.74 & -0.24728 & -0.70084 \\
3.63661 & -0.41975 & -1.77675 \\
3.27411 & 0.71414 & -0.46491 \\
4.80813 & -0.17526 & -0.46935 \\
3.26964 & -1.40333 & 2.13466 \\
2.87311 & -0.44208 & 2.47703 \\
2.79124 & -2.19238 & 2.72457 \\
4.34349 & -1.42581 & 2.35132 \\
3.61096 & -3.32417 & -0.26179 \\
4.6905 & -3.38919 & -0.08695 \\
3.13731 & -4.14248 & 0.29179 \\
3.42924 & -3.48812 & -1.32865 \\
0.09098 & 4.16578 & 2.37978 \\
-0.71155 & 2.58672 & 2.41115 \\
1.05067 & 2.68724 & 2.55761 \\
2.00278 & 3.78883 & -1.49788 \\
1.82726 & 4.97051 & -0.18907
\end{array}
$$

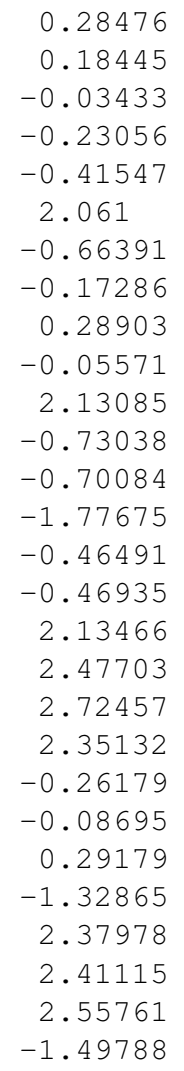

$\begin{array}{rcc}2.78357 & 3.50209 & 0.06648 \\ -1.11759 & 3.59492 & -1.75153 \\ -2.09555 & 3.14074 & -0.34552 \\ -1.34516 & 4.74385 & -0.42173 \\ -5.50612 & -0.39614 & -0.47939 \\ -4.326 & -0.32759 & -1.80002 \\ -4.3264 & 0.92348 & -0.54586 \\ -3.33357 & -3.23327 & -1.1166 \\ -2.78192 & -3.61412 & 0.52432 \\ -4.50267 & -3.33996 & 0.21051 \\ -2.71591 & -1.3736 & 2.71617 \\ -4.44831 & -1.08231 & 2.48455 \\ -3.29736 & 0.2559 & 2.3356 \\ 0.01029 & -0.40966 & -2.7161 \\ 1.01236 & -0.17795 & -3.09655 \\ -0.26749 & -1.41481 & -3.05554 \\ -0.70106 & 0.31343 & -3.13634 \\ 0.73622 & -2.5027 & 0.17366\end{array}$

\section{$\mathrm{R}=\mathrm{Et}, \mathrm{H}=-714.019436$}

Ti, $0.0911834556,0.61242099,-0.1638576628$ $\mathrm{Si}, 3.3892185777,-0.1401365945,-0.2158359406$ Si, $-0.9036754444,-1.0847412457,2.6610138581$ $\mathrm{N}, 1.7993881689,0.2650167413,-0.8913205156$ $0,-0.1790830901,-0.1983797387,1.4130229826$ C, $-0.3474818522,-0.3347685807,4.3003135511$ C, $-0.3319485955,-2.8779184067,2.5169999301$ $C,-2.7797822831,-0.9724104635,2.4803701276$ $0,-1.1604773991,0.3934619892,-1.420913865$ Si, $-2.3181354198,-0.0266284674,-2.5831544167$ C, $-1.7730419794,0.7107378685,-4.2326500729$ $C,-2.3979615606,-1.9096699231,-2.6841685534$ $C,-3.9800015816,0.6865009934,-2.0470595285$ C, $3.426233908,0.4104537926,1.5898398629$ $\mathrm{H}, 3.3491552456,1.4995070116,1.6683401846$ $\mathrm{H}, 2.5933834766,-0.0287111631,2.1469905371$ $\mathrm{H}, 4.360226628,0.1006586596,2.0708150187$ C, $3.7004490765,-2.0061888461,-0.329439958$ $\mathrm{H}, 2.9422997121,-2.5585994964,0.2347932671$ $\mathrm{H}, 3.6585418272,-2.3478883008,-1.3691796846$ $\mathrm{H}, 4.6850250235,-2.2715897233,0.0716799448$ $\mathrm{C}, 4.7152535668,0.7725269427,-1.216002305$ $\mathrm{H}, 5.7177862065,0.5447906604,-0.8374945168$ $\mathrm{H}, 4.6876014922,0.4823025577,-2.2721602727$ $\mathrm{H}, 4.5684592157,1.855731411,-1.1596567715$ $\mathrm{H},-0.7699064667,-3.4931415462,3.3106184366$ $\mathrm{H},-0.6284916634,-3.3065466722,1.5544008622$ $\mathrm{H}, 0.7572714014,-2.9477718506,2.5980387615$ $\mathrm{H},-0.6441167782,0.716565366,4.367592264$ $\mathrm{H},-0.7944709633,-0.8680710642,5.1464406852$ $\mathrm{H}, 0.7406814153,-0.3861986411,4.405422003$ $\mathrm{H},-3.1174547679,0.0670054821,2.5399170225$ $\mathrm{H},-3.1039299957,-1.3808659765,1.5179834446$ $\mathrm{H},-3.282383175,-1.5372781063,3.2731971994$ $\mathrm{H},-4.762427653,0.4457664221,-2.7750454461$ $\mathrm{H},-3.9254802869,1.7758472361,-1.9571411759$ $\mathrm{H},-4.2841359442,0.280865373,-1.0771244348$ $\mathrm{H},-1.6701262513,1.797690312,-4.1553193991$ $\mathrm{H},-0.8082110428,0.2992080892,-4.5460232406$ $\mathrm{H},-2.5028794176,0.4946556643,-5.020471259$ $\mathrm{H},-1.4255111212,-2.3275928787,-2.963356346$ $\mathrm{H},-3.1319118665,-2.231477688,-3.4310765439$ $\mathrm{H},-2.6875063141,-2.339503091,-1.7200075165$ C, $0.1018503123,2.6584294067,0.251890702$ 
$\mathrm{H}, 0.9485507968,2.8342417754,0.9311972981$ $\mathrm{H}, 0.3445331014,3.1690286226,-0.6915144332$ $\mathrm{H}, 1.8034334825,0.2935084924,-1.9104384965$ C, $-1.2006212208,3.1941940357,0.8477234793$ $\mathrm{H},-1.1538280231,4.2741964458,1.0468056883$ $\mathrm{H},-2.0503874753,3.0297711637,0.1751187886$ $\mathrm{H},-1.440453843,2.70462587,1.7986649565$

\section{$\mathrm{R}=\mathrm{Pr}, \mathrm{H}=-753.292122$}

Ti, $0.0154595183,-0.2772433082,0.3974576419$ Si, $-2.992561268,-1.7274712411,-0.1703438948$ $\mathrm{Si},-0.4815260924,2.8554920047,-0.930758493$ $\mathrm{N},-1.2356293358,-1.652614142,0.0675518365$ $0,-0.5188913586,1.3215366708,-0.2124855079$ C, $-1.7042177156,3.9548211752,-0.0057521272$ C, $-0.9840660351,2.6663438122,-2.7402276678$ C, $1.2686891324,3.5502898187,-0.7952896601$ $0,1.6531655003,-0.8188090239,-0.0714896582$ $\mathrm{Si}, 3.1020673129,-1.3506910048,-0.7681407183$ C, $3.0997970832,-3.2381022148,-0.7453870603$ C, $3.1820039169,-0.7057632968,-2.5402105533$ $C, 4.5281524365,-0.6689440086,0.2603804179$ $C,-3.7547836625,-0.1404929561,0.5107230862$ $\mathrm{H},-3.6155864375,-0.0723569102,1.5942709707$ $\mathrm{H},-3.2917135958,0.7392546394,0.0544779172$ $\mathrm{H},-4.8298384237,-0.1081751974,0.3036273466$ C, $-3.4004683531,-1.9017498706,-2.0130422959$ $\mathrm{H},-3.0146290932,-1.0460942837,-2.5764399173$ $\mathrm{H},-2.9496559831,-2.8083061057,-2.430867678$ $\mathrm{H},-4.4822484348,-1.958328751,-2.178273122$ $C,-3.6606890281,-3.2368752031,0.7606389305$ $\mathrm{H},-4.7468021425,-3.3213518343,0.6444366729$ $\mathrm{H},-3.2155737317,-4.1655805191,0.3867841996$ $\mathrm{H},-3.4384547349,-3.162754903,1.8298826279$ $\mathrm{H},-0.9866357831,3.6360154586,-3.2499722907$ $\mathrm{H},-0.2901512757,2.0069451978,-3.2711978134$ $\mathrm{H},-1.9882505691,2.2389313121,-2.8228678429$ $\mathrm{H},-1.4376011919,4.0255663495,1.0534301172$ $\mathrm{H},-1.7117595137,4.9681950199,-0.4218945101$ $\mathrm{H},-2.7207012145,3.5548013289,-0.0724561169$ $\mathrm{H}, 1.5747180573,3.6347521544,0.2521487511$ $\mathrm{H}, 1.9872992859,2.9032847963,-1.3080334922$ $\mathrm{H}, 1.3293090803,4.5466502276,-1.2468008235$ $\mathrm{H}, 5.4937573259,-0.9916950595,-0.1439329596$ $\mathrm{H}, 4.4609478233,-1.0171631869,1.2957460912$ $\mathrm{H}, 4.5134649646,0.4254241208,0.2691486189$ $\mathrm{H}, 3.0132087489,-3.6149436097,0.2787060705$ $\mathrm{H}, 2.2620031395,-3.6351061359,-1.3277381546$ $\mathrm{H}, 4.0256832371,-3.6363554758,-1.1745319641$ $\mathrm{H}, 2.3316236968,-1.0683569745,-3.1264564912$ $\mathrm{H}, 4.1010788998,-1.0360295524,-3.0365384493$ $\mathrm{H}, 3.1650692521,0.3884870669,-2.5572336529$ C, $0.0666019697,-0.1018787964,2.4781909034$ $\mathrm{H},-0.9646843363,0.1075278609,2.8017458394$ $\mathrm{H}, 0.32138467,-1.0990320086,2.8699314539$ $\mathrm{H},-0.7932204707,-2.568009529,-0.0111112619$ $C, 1.0312423141,0.9552396383,3.0197514453$ $\mathrm{H}, 2.0485907013,0.7481766813,2.6605007743$ $\mathrm{H}, 0.7643557923,1.9412011406,2.6152135649$ C, $1.0541901722,1.0396205543,4.5493743226$ $\mathrm{H}, 1.7551425546,1.805032361,4.9034044748$ $\mathrm{H}, 0.0613048148,1.2860566281,4.9426622286$ $\mathrm{H}, 1.3532349839,0.0814997549,4.9896042097$

$\mathrm{R}=c-\mathrm{Pr}, \mathrm{H}=-752.071620$
Ti, $0.0754571236,0.1102271961,0.541743477$ Si, $-1.3676408689,3.1138613453,-0.06070261$ Si, $3.2700048829,-0.8240252016,-0.3530446737$ $\mathrm{N},-0.0791423335,1.986221379,0.404432734$ $0,1.7836197767,-0.3872293238,0.3329465533$ C, $4.5931037232,0.3159320064,0.3621446404$ C, $3.1483887482,-0.6136349381,-2.2249230523$ C, 3.6213592918,-2.6212511823,0.1002775977 $0,-1.1473522414,-0.727439365,-0.4629792866$ $\mathrm{Si},-2.0008292171,-1.966938465,-1.2413889665$ C, $-3.5863969497,-2.2830968737,-0.2701085917$ $C,-2.3887134504,-1.3849395617,-2.9943769579$ C, $-0.9244074518,-3.5174238006,-1.2761851098$ C, $-3.0364676177,2.3061048829,0.2983026342$ $\mathrm{H},-3.1156141357,1.3430792366,-0.2147192185$ $\mathrm{H},-3.1622213233,2.1307726958,1.371407166$ $\mathrm{H},-3.8597486142,2.9432161456,-0.0424338925$ C, $-1.161249395,4.6966877661,0.9609050622$ $\mathrm{H},-1.2309577074,4.4806502104,2.0317776517$ $\mathrm{H},-0.1900326,5.1678521536,0.7731153771$ $\mathrm{H},-1.9367516199,5.4290327936,0.7110845088$ C, $-1.2473354273,3.5357529972,-1.9040477923$ $\mathrm{H},-2.0351574728,4.2351369677,-2.2056732045$ $\mathrm{H},-0.2810909562,3.9945067385,-2.1391436659$ $\mathrm{H},-1.3454171874,2.6302784992,-2.5115579155$ $\mathrm{H}, 4.1012894242,-0.8522793212,-2.7098696255$ $\mathrm{H}, 2.3824106869,-1.2741531942,-2.6433820951$ $\mathrm{H}, 2.8852481599,0.4170842616,-2.4834524789$ $\mathrm{H}, 4.6393869014,0.2235536929,1.4518081646$ $\mathrm{H}, 5.581537664,0.0688909013,-0.0403879422$ $\mathrm{H}, 4.3821900772,1.3621400027,0.1185457136$ $\mathrm{H}, 3.6712204163,-2.7464321526,1.1864614073$ $\mathrm{H}, 2.8359763307,-3.2808990167,-0.2816907817$ $\mathrm{H}, 4.5762995189,-2.9521964365,-0.3224339011$ $\mathrm{H},-1.444970731,-4.3422463468,-1.7749884953$ $\mathrm{H},-0.6754628051,-3.8397037229,-0.2601544774$ $\mathrm{H}, 0.0102889362,-3.3335557394,-1.8152178063$ $\mathrm{H},-3.3550757385,-2.5829194942,0.7567532928$ $\mathrm{H},-4.2071810938,-1.3827566143,-0.2284355351$ $\mathrm{H},-4.1776015965,-3.0809264692,-0.7328620113$ $\mathrm{H},-3.0048876067,-0.4805234241,-2.9769919984$ $\mathrm{H},-2.9337920036,-2.1544717449,-3.5519034217$ $\mathrm{H},-1.4689449536,-1.1574129454,-3.5424814058$ $\mathrm{H}, 0.7853099915,2.46358139,0.6579126267$ C, $-0.3735103161,-0.347512073,2.5002959728$ C, $0.1472234583,-1.6195913585,3.1456615544$ C, $0.6707858464,-0.2887469893,3.5968393427$ $\mathrm{H},-1.3770953412,-0.0560212321,2.8184828011$ $\mathrm{H}, 0.8140275024,-2.2614525723,2.574018556$ $\mathrm{H},-0.5150596159,-2.1633250888,3.8161069732$ $\mathrm{H}, 0.3658997606,0.0761627856,4.575298908$ $\mathrm{H}, 1.6916071526,-0.0261141688,3.3290786614$

\section{$\mathrm{R}=c-\mathrm{Pe}, \mathrm{H}=-830.643738$}

Ti, $-0.0469460992,0.2019135567,0.116382314$ Si, 3.1777595515,-0.5194515972,-0.7106838052 $\mathrm{Si},-1.2949474256,-0.841502497,3.1689680107$ $\mathrm{N}, 1.8359706702,0.0951883871,0.27554935$ $0,-0.8354772898,-0.1877338401,1.677185204$ C, $0.0634115369,-0.4612657444,4.4240877988$ $C_{,}-1.5020559789,-2.7062620606,2.9634447625$ C, $-2.9221780418,-0.0345327976,3.6776665893$ $0,-0.6764277502,-0.6519762845,-1.331351075$ Si, $-1.8710181456,-1.4117782643,-2.2643163136$ C, $-1.5596078253,-0.9682010219,-4.0711612074$ 
$C,-1.7289229859,-3.2742204416,-1.9934569265$ $C_{,}-3.5628296604,-0.7827749191,-1.7101718728$ C, $2.6964414461,-0.3949864785,-2.5306180398$ $\mathrm{H}, 1.7358877612,-0.8876810023,-2.7060692736$ $\mathrm{H}, 2.6023921214,0.6493696268,-2.8435666496$ $\mathrm{H}, 3.4510608935,-0.8720393081,-3.1652175366$ C, $4.7158527789,0.5279157877,-0.3511192857$ $\mathrm{H}, 4.5363296102,1.5802276953,-0.5928272909$ $\mathrm{H}, 4.9996952367,0.4680485319,0.7055226759$ $\mathrm{H}, 5.5720959733,0.1850408859,-0.9422713751$ C, $3.5431102141,-2.3267593266,-0.2680405867$ $\mathrm{H}, 4.3802964674,-2.720877161,-0.8550548988$ $\mathrm{H}, 3.8005200037,-2.4275891351,0.7918332195$ $\mathrm{H}, 2.6676080746,-2.9551855971,-0.4614710019$ $\mathrm{H},-1.7742724935,-3.1789025991,3.9134823737$ $\mathrm{H},-2.2869530588,-2.9352431546,2.2356998129$ $\mathrm{H},-0.5703571526,-3.1618986252,2.6131577669$ $\mathrm{H}, 0.2167372567,0.6186322482,4.5165933227$ $\mathrm{H},-0.1967609901,-0.8514211817,5.4140846362$ $\mathrm{H}, 1.0125187321,-0.91667052,4.1231102662$ $\mathrm{H},-2.8058781292,1.0497961012,3.7691391149$ $\mathrm{H},-3.7022054168,-0.2293972221,2.9350038656$ $\mathrm{H},-3.2687903259,-0.4214295043,4.6421445195$ $\mathrm{H},-4.3654210779,-1.2579992952,-2.2848186589$ $\mathrm{H},-3.6409895973,0.2997208855,-1.8529027927$ $\mathrm{H},-3.7324778742,-0.998645332,-0.650510903$ $\mathrm{H},-1.6128147715,0.1149993204,-4.2178784948$ $\mathrm{H},-0.5698306128,-1.305344666,-4.3939992237$ $\mathrm{H},-2.3056479016,-1.4358824798,-4.7229817605$ $\mathrm{H},-0.7378396983,-3.6356067524,-2.2853134554$ $\mathrm{H},-2.4747770648,-3.8163107104,-2.5848967133$ $\mathrm{H},-1.8844412021,-3.5260928291,-0.9396698531$ $\mathrm{H}, 2.1373713827,0.329049692,1.2221625209$ C, $-0.4887705732,2.2164404546,-0.1710179784$ C, $-0.0417630905,3.1690334754,0.9533879393$ $C,-0.048428521,4.5494685686,0.280691046$ C, $0.5541736081,4.2676565207,-1.1011657633$ C, $0.0652360301,2.8429767038,-1.4782094757$ $\mathrm{H},-1.5916276,2.2220157313,-0.2063023536$ $\mathrm{H}, 0.9820846968,2.9386050086,1.282886788$ $\mathrm{H},-0.6867987918,3.1108060342,1.8363876226$ $\mathrm{H},-1.0847735183,4.8972205607,0.1774831819$ $\mathrm{H}, 0.5021978906,5.3143543761,0.8404293589$ $\mathrm{H}, 0.2764275111,5.0189208073,-1.848074422$ $\mathrm{H}, 1.6485727673,4.2791526614,-1.0295059422$ $\mathrm{H},-0.7131938105,2.8749159045,-2.2487729113$ $\mathrm{H}, 0.893185174,2.2639257377,-1.9043974092$

\section{$\mathrm{R}=c-\mathrm{Bu}, \mathrm{H}=-791.343003$}

Ti, $-0.0960942693,0.4008816832,-0.0395524614$ $\mathrm{Si}, 3.2284874936,-0.293913736,-0.2111500248$ $\mathrm{Si},-1.0933061727,-1.5600145599,2.5726777396$ $\mathrm{N}, 1.6599552588,0.3467951968,-0.7422943673$ $0,-0.2795737032,-0.5955906675,1.4407704055$ $C_{,}-0.4755157621,-1.0787139983,4.288598682$ C, $-0.6926363431,-3.3656310365,2.1969676257$ C, $-2.9469794314,-1.2376159965,2.4173700367$ $0,-1.302140517,0.1407956339,-1.3368836837$ Si, $-2.2732042522,-0.2939760634,-2.6523181469$ C, $-1.4591130391,0.3587750975,-4.2258139199$ C, $-2.3970421061,-2.176922052,-2.7020500013$ C, $-3.9719645298,0.4870825961,-2.4045224831$ $\mathrm{C}, 3.3266747582,-0.1826929585,1.670226366$ $\mathrm{H}, 3.3323709092,0.8607470056,2.0000401169$
$\mathrm{H}, 2.4689940111,-0.6798530678,2.1324990778$ $\mathrm{H}, 4.2406212409,-0.6604896707,2.0392666276$ C, $3.4202503437,-2.0990277455,-0.757428167$ $\mathrm{H}, 2.6282215062,-2.7180916561,-0.3236034959$ $\mathrm{H}, 3.3563327175,-2.1886302492,-1.8471631317$ $\mathrm{H}, 4.385194009,-2.5119615637,-0.4425387393$ C, $4.5978647991,0.7460930207,-1.0083354321$ $\mathrm{H}, 5.5881233387,0.3789877599,-0.7170573782$ $\mathrm{H}, 4.5417271619,0.7106781144,-2.1019350642$ $\mathrm{H}, 4.5222502287,1.7936894558,-0.7000715141$ $\mathrm{H},-1.1925740627,-4.0351595137,2.9053801113$ $\mathrm{H},-1.0187429272,-3.6360201389,1.1876476685$ $\mathrm{H}, 0.3847351505,-3.5464885603,2.2647408264$ $\mathrm{H},-0.6678020606,-0.0195974223,4.4864102923$ $\mathrm{H},-0.9782651687,-1.6659260945,5.0648061945$ $\mathrm{H}, 0.6015530946,-1.2499912875,4.3797947738$ $\mathrm{H},-3.177119841,-0.1875073312,2.6238966927$ $\mathrm{H},-3.2985579422,-1.4696720745,1.4071510792$ $\mathrm{H},-3.5146540828,-1.8532691534,3.1236944058$ $\mathrm{H},-4.6420138377,0.2441295825,-3.2364066568$ $\mathrm{H},-3.8910085587,1.5766871014,-2.340731053$ $\mathrm{H},-4.4355066409,0.1276534302,-1.4804523984$ $\mathrm{H},-1.3484547599,1.4469778578,-4.1841994037$ $\mathrm{H},-0.4658672713,-0.080732936,-4.3636518075$ $\mathrm{H},-2.0595674819,0.1142995629,-5.1088401866$ $\mathrm{H},-1.4049574078,-2.6289921233,-2.8008593412$ $\mathrm{H},-3.0060939983,-2.5082749917,-3.5501993989$ $\mathrm{H},-2.8562497869,-2.5622903558,-1.7862601946$ $\mathrm{H}, 1.6788274155,0.6291512823,-1.722583319$ C, $-0.4568908102,2.3592803955,0.5595429853$ C, $0.5551675483,3.0171810241,1.5355862121$ C, $1.1319927861,3.8188996583,0.3462033967$ $C,-0.1334647766,3.4745456084,-0.4718915928$ $\mathrm{H},-1.5016740434,2.3257705781,0.9077979272$ $\mathrm{H}, 1.2487216408,2.3685412791,2.0827468614$ $\mathrm{H}, 0.0591981269,3.6671653176,2.2683866315$ $\mathrm{H}, 2.0219593077,3.3380014004,-0.0689296195$ $\mathrm{H}, 1.3498590837,4.878684371,0.5121772239$ $\mathrm{H}, 0.0140451177,3.1910157028,-1.5214979689$ $\mathrm{H},-0.8732628973,4.2853045985,-0.4499910916$

\section{$\mathrm{R}=\mathrm{Cy}, \mathrm{H}=-869.923656$}

Ti, $-0.0938875693,-0.0593744438,-0.088269499$ $\mathrm{Si},-0.5565152626,-2.178049241,2.5375370953$ $\mathrm{Si},-0.9838168936,-0.7761386229,-3.3718979547$ $\mathrm{N},-0.9570146016,-1.3013978288,1.0470144045$ $0,-0.5638681234,-0.3341180931,-1.7935390178$ $C,-2.7449703795,-1.4558887814,-3.3424957886$ C, $0.226333781,-2.0980834514,-3.9632752498$ $C,-0.8803688037,0.7654844057,-4.4542423578$ $0,1.6630939776,0.1079662942,0.2401158885$ $\mathrm{Si}, 3.3002023602,0.3223763912,-0.1435142179$ C, $4.1797844274,0.9332944478,1.40957441$ C, $4.0006556547,-1.340647701,-0.6973872191$ C, $3.4316140562,1.5998446254,-1.5272891377$ C, $0.7607620985,-1.2049984148,3.4739739825$ $\mathrm{H}, 1.6236102804,-1.0077956601,2.8314477782$ $\mathrm{H}, 0.368839515,-0.2424624235,3.8164310569$ $\mathrm{H}, 1.1045996132,-1.7641295667,4.3507983315$ C, $-2.1308533482,-2.3418651684,3.5801645381$ $\mathrm{H},-2.5329125342,-1.3559883664,3.8341340999$ $\mathrm{H},-2.9088295404,-2.8963722745,3.0438055724$ $\mathrm{H},-1.9289953355,-2.8771195862,4.5145616826$ C, $0.0955113502,-3.9098713871,2.1242131324$ 
$\mathrm{H}, 0.3367941296,-4.4722279194,3.0331974937$ $\mathrm{H},-0.6465743718,-4.486020992,1.5610961677$ $\mathrm{H}, 1.0022499033,-3.8444670308,1.5140371835$ $\mathrm{H},-0.022998259,-2.4313600324,-4.9766266217$ $\mathrm{H}, 1.2511375219,-1.7138494014,-3.9777595227$ $\mathrm{H}, 0.199758037,-2.9712633544,-3.3036498749$ $\mathrm{H},-3.4467978965,-0.7053720476,-2.9652439957$ $\mathrm{H},-3.0700613397,-1.7465273773,-4.3474656004$ $\mathrm{H},-2.8124243597,-2.3393600479,-2.699374874$ $\mathrm{H},-1.5623127485,1.5404556997,-4.0906034075$ $\mathrm{H}, 0.1335315994,1.177709982,-4.4491954478$ $\mathrm{H},-1.1481380363,0.5372998471,-5.49164512$ $\mathrm{H}, 4.4773423364,1.7755078372,-1.8024359892$ $\mathrm{H}, 2.9992242044,2.5557453875,-1.2149397541$ $\mathrm{H}, 2.8985836412,1.2627434155,-2.4219302212$ $\mathrm{H}, 3.7490276833,1.8784095033,1.7545563514$ $\mathrm{H}, 4.0923457409,0.2053591726,2.2220682833$ $\mathrm{H}, 5.2455569759,1.0973888125,1.2162848517$ $\mathrm{H}, 3.9063848072,-2.0862454864,0.0983967487$ $\mathrm{H}, 5.0618381849,-1.2538328638,-0.9552419529$ $\mathrm{H}, 3.4681650727,-1.7155180199,-1.5771685731$ $\mathrm{H},-1.8327775136,-1.6278208783,0.6372747148$ $C_{,}-3.0151865629,3.2712925672,0.3579291701$ $C_{,}-2.4403918846,1.8715976004,0.0910938049$ $C,-0.9310800102,1.8061851241,0.359071722$ C, $-0.605694585,2.3025670425,1.7732374602$ C, $-1.1771206876,3.7036907231,2.0386825529$ C, $-2.6832354717,3.7511451535,1.7724790018$ $\mathrm{H},-2.955917793,1.1511682638,0.7448451269$ $\mathrm{H},-2.6642430205,1.571327711,-0.9400915488$ $\mathrm{H},-2.5926124051,3.9771668831,-0.3720123944$ $\mathrm{H},-4.1021231307,3.2713517977,0.2013089316$ $\mathrm{H},-1.0345571356,1.604694934,2.5077818731$ $\mathrm{H}, 0.4780166089,2.3049546535,1.9436060357$ $\mathrm{H},-0.9631310535,4.0117870631,3.0707343897$ $\mathrm{H},-0.6708726706,4.4277526853,1.3835909296$ $\mathrm{H},-3.1973514716,3.1062573088,2.5006863596$ $\mathrm{H},-3.0678172136,4.7665229678,1.9312629265$ $\mathrm{H},-0.4174767866,2.4622462643,-0.3704378583$

\section{$\mathrm{R}=$ Benzyl, $\mathrm{H}=--905.634055$}

Ti, $-0.5902047175,0.0111063308,0.2517180808$ $\mathrm{Si},-1.7207288954,0.5647161025,3.3833690195$ $\mathrm{Si}, 2.7400685907,0.6822998641,0.6483953954$ $\mathrm{N},-1.8063346409,0.484171261,1.6101526831$ $0,1.1022173827,0.2456900555,0.7790167627$ C, $2.8776418996,2.5269656296,1.0282220413$ C, $3.3331268925,0.3059109194,-1.0991928825$ C, $3.6964904457,-0.330235187,1.9209276143$ $0,-1.11084456,0.7798177173,-1.2728240679$ $\mathrm{Si},-1.525159426,1.7525037331,-2.5987882342$ C, $-2.8706246975,0.8553211153,-3.5707310023$ C, $-2.1626391435,3.4000435712,-1.9348606191$ C, $0.0119878187,2.0038418836,-3.6610484815$ C, $-0.4614416804,-0.711660393,3.9739449571$ $\mathrm{H},-0.8008649718,-1.7264664577,3.7432988656$ $\mathrm{H}, 0.5058855791,-0.5568901632,3.4864459579$ $\mathrm{H},-0.3127082335,-0.6417592194,5.0567953023$ C, $-1.1987700399,2.2990199689,3.9367258843$ $\mathrm{H},-0.2190566714,2.553467866,3.5201145526$ $\mathrm{H},-1.9151675753,3.052012402,3.5914967879$ $\mathrm{H},-1.134719316,2.3703370481,5.0282378376$ $C,-3.441128718,0.1737799891,4.0737212531$ $\mathrm{H},-3.4400052224,0.2084082867,5.1686392638$
$\mathrm{H},-4.1850293198,0.8971962165,3.7219713513$ $\mathrm{H},-3.7683693982,-0.8247079612,3.766877665$ $\mathrm{H}, 4.3991828216,0.534801116,-1.2070182769$ $\mathrm{H}, 3.1816735025,-0.7515702039,-1.3357914078$ $\mathrm{H}, 2.7834889233,0.8999036287,-1.8358236259$ $\mathrm{H}, 2.5051667071,2.749014418,2.0331098863$ $\mathrm{H}, 3.9200413984,2.8596061888,0.9733461822$ $\mathrm{H}, 2.2973474674,3.118873321,0.3131375181$ $\mathrm{H}, 3.3331676285,-0.1313581394,2.9339943332$ $\mathrm{H}, 3.5894529942,-1.4012442869,1.7239745834$ $\mathrm{H}, 4.7638982035,-0.0855441044,1.8913473087$ $\mathrm{H},-0.2240056189,2.5883086379,-4.5570690599$ $\mathrm{H}, 0.4212198461,1.0407008402,-3.9810354389$ $\mathrm{H}, 0.7897087221,2.5380906875,-3.1063598908$ $\mathrm{H},-2.5001514053,-0.098505591,-3.9585741369$ $\mathrm{H},-3.7403578316,0.6502603614,-2.9385861517$ $\mathrm{H},-3.2056932986,1.4580985381,-4.4219284883$ $\mathrm{H},-3.0514114236,3.2550252601,-1.3124798937$ $\mathrm{H},-2.4326738198,4.0737041574,-2.755438255$ $\mathrm{H},-1.3999428235,3.8966156537,-1.326717561$ C, $-0.8806908865,-2.0667999174,0.004955838$ $\mathrm{H},-0.8109082023,-2.5014568614,1.0092389139$ $\mathrm{H},-1.9161550456,-2.169810798,-0.3441424012$ $\mathrm{H},-2.6961193154,0.8195606842,1.2432499077$ C, $0.097779847,-2.6687656325,-0.9377173342$ C, $-0.1294548875,-2.6768487415,-2.3258254193$ C, $0.7998320831,-3.2182294197,-3.2104133794$ C, $1.9888819483,-3.7711295852,-2.7351425809$ C, $2.2305224478,-3.7789115079,-1.3608323107$ C, $1.3006075028,-3.2356859817,-0.4781472308$ $\mathrm{H},-1.0572276581,-2.2583224776,-2.7093294073$ $\mathrm{H}, 0.5902604248,-3.2153197836,-4.2774333652$ $\mathrm{H}, 2.7124557221,-4.1977693366,-3.4241020122$ $\mathrm{H}, 3.1459517778,-4.2189978223,-0.9725109766$ $\mathrm{H}, 1.4982803574,-3.2548384531,0.5914713991$

\section{$\mathrm{R}=\mathrm{CH}_{2} \mathrm{SiMe}_{3}, \mathrm{H}=-797.690594$}

Ti, $-0.1220542076,0.2142554454,-0.3644313052$ $\mathrm{Si},-3.3340532161,0.6834829924,-1.3067223654$ Si, $-0.7268817282,-1.0593954592,2.7433407893$ $\mathrm{N},-1.6244397674,1.1007423097,-1.0890070437$ $0,-0.5531924878,-0.7540580715,1.083929949$ C, $-2.0086839046,-2.4287262777,2.9455475653$ C, $-1.30478207,0.5264454773,3.5899812741$ C, $0.9418824798,-1.6048117016,3.435224494$ $0,1.1670256076,1.4232505774,-0.1343225635$ Si,2.2591492111,2.6920277151,0.1344955897 C, $3.2983999268,2.9070746205,-1.4245639825$ C, $1.26055761,4.2541912954,0.4902283341$ C, $3.3383200443,2.2351655546,1.6119537385$ $C,-3.5161312868,-1.1850251264,-1.1054808724$ $\mathrm{H},-3.0106392635,-1.7158863679,-1.9183511308$ $\mathrm{H},-3.0824809641,-1.5222278932,-0.1591536541$ $\mathrm{H},-4.572317811,-1.4748095185,-1.1180494618$ $C,-4.4031912806,1.5818205363,-0.0252171358$ $\mathrm{H},-4.1181845529,1.289570458,0.9903846481$ $\mathrm{H},-4.2838877165,2.6675555378,-0.1063880293$ $\mathrm{H},-5.4656574358,1.3500028961,-0.1599503668$ $C,-3.8712349617,1.2196058851,-3.0431338467$ $\mathrm{H},-4.9257293491,0.9808951373,-3.2201637782$ $\mathrm{H},-3.7496361031,2.2998332006,-3.1805331381$ $\mathrm{H},-3.2747518255,0.7128421072,-3.8083357599$ $\mathrm{H},-1.4580563856,0.3629002786,4.6624253522$ $\mathrm{H},-0.5643789301,1.3242033498,3.4731227416$ 
$\mathrm{H},-2.2495424868,0.8759808018,3.1624671055$ $\mathrm{H},-1.7055720488,-3.3290285345,2.4021696261$ $\mathrm{H},-2.1298099048,-2.6952775622,4.0012282642$ $\mathrm{H},-2.984881411,-2.1140453679,2.5637489552$ $\mathrm{H}, 1.2914326616,-2.5183971804,2.9447336099$ $\mathrm{H}, 1.6989623351,-0.8284951283,3.2863701405$ $\mathrm{H}, 0.8694975209,-1.8045119622,4.5100564435$ $\mathrm{H}, 4.0702437347,3.0225442482,1.8226512493$ $\mathrm{H}, 3.8845527132,1.3067419945,1.4195540434$ $\mathrm{H}, 2.7290402185,2.0928050186,2.5100957122$ $\mathrm{H}, 3.8604214587,1.9952011418,-1.647938229$ $\mathrm{H}, 2.6648108349,3.1349366546,-2.2876284572$ $\mathrm{H}, 4.0154789245,3.7266458496,-1.3054982467$ $\mathrm{H}, 0.6326882787,4.522722065,-0.3654257463$ $\mathrm{H}, 1.9236033641,5.1009584362,0.6988080704$ $\mathrm{H}, 0.6088994719,4.1133529923,1.3582525445$ C, $0.5389110048,-1.0907538312,-1.8454377175$ $\mathrm{H},-0.3558735029,-1.6001346046,-2.2341043316$ $\mathrm{H}, 0.9018135869,-0.4324488523,-2.6505453877$ $\mathrm{H},-1.3963018078,2.0282356067,-1.444285943$ Si, $1.8479123448,-2.3954745745,-1.4517227497$ C, $3.2838616145,-1.6523175901,-0.4526978746$ $\mathrm{H}, 4.0492981659,-2.4073122355,-0.2419545061$ $\mathrm{H}, 3.7612262211,-0.8360079267,-1.0052126834$ $\mathrm{H}, 2.931843421,-1.2507202415,0.5027448297$ C, $1.0590340351,-3.8111778122,-0.4638322385$ $\mathrm{H}, 1.8026293596,-4.5615069939,-0.1745206298$ $\mathrm{H}, 0.5850853715,-3.4274918529,0.444462161$ $\mathrm{H}, 0.2874967235,-4.3149265598,-1.0562721773$ C, $2.5680464488,-3.1115110836,-3.0630258984$ $\mathrm{H}, 3.0499175902,-2.3295695286,-3.6600312436$ $\mathrm{H}, 3.3170809176,-3.8842574768,-2.8561917326$ $\mathrm{H}, 1.7815740829,-3.5620771107,-3.6778146609$

\section{$\mathrm{R}=$ Methallyl, $\mathrm{H}=-791.359113$}

Ti, $0.1415751688,-0.5421935333,-0.0216841766$ $\mathrm{Si},-0.4861422853,-1.1138037339,-3.296009406$ $\mathrm{Si},-1.9665223611,2.0884280313,0.502765634$ $\mathrm{N}, 0.4899610459,-0.9925608716,-1.8179403291$ $0,-1.2274140984,0.6100671929,0.1167459222$ C, $-0.9878507139,3.475277037,-0.3241329422$ C, $-1.9618281204,2.2951701881,2.3770423835$ $C,-3.7325652613,2.0350354232,-0.1594613487$ $0,1.6968402256,-0.0956886875,0.7317006593$ $\mathrm{Si}, 3.0946061769,0.540480513,1.449087921$ C, $4.2013242176,-0.9078330339,1.9348878833$ C, $3.9521066932,1.6402830681,0.1771215621$ C, $2.5847761382,1.5365902991,2.9669441738$ C, $-2.297849748,-1.2901444917,-2.7972523586$ $\mathrm{H},-2.4708517163,-2.2359622877,-2.2741894627$ $\mathrm{H},-2.597487854,-0.4749361493,-2.1320902479$ $\mathrm{H},-2.9462892958,-1.267818693,-3.679619784$ C, $-0.2550111134,0.4346326379,-4.3632654159$ $\mathrm{H},-0.57863527,1.3288182063,-3.821120289$ $\mathrm{H}, 0.79747213,0.5702948315,-4.6341391406$ $\mathrm{H},-0.8349576797,0.3707731768,-5.2907733486$ C, $0.0882716382,-2.6336648716,-4.2707178708$ $\mathrm{H},-0.4962239145,-2.7538329969,-5.1895151228$ $\mathrm{H}, 1.1417805429,-2.5478033118,-4.5589191157$ $\mathrm{H},-0.025758059,-3.544559924,-3.6745833007$ $\mathrm{H},-2.4506651447,3.2314646376,2.6681671264$ $\mathrm{H},-2.4919126176,1.4694050874,2.8607811082$ $\mathrm{H},-0.9389494947,2.3109595934,2.765414315$ $\mathrm{H},-0.9722244376,3.34977002,-1.4112136366$
$\mathrm{H},-1.4307603577,4.4525404782,-0.1027239169$ $\mathrm{H}, 0.0477610703,3.486244373,0.0305132847$ $\mathrm{H},-3.7416193902,1.9119677043,-1.24693268$ $\mathrm{H},-4.2909764187,1.2032337135,0.2810820756$ $\mathrm{H},-4.2645103067,2.9625855573,0.0789035689$ $\mathrm{H}, 3.4626473237,1.9195033953,3.4987581784$ $\mathrm{H}, 2.0105830158,0.9132426677,3.6591908278$ $\mathrm{H}, 1.9633179562,2.3919155492,2.6836096166$ $\mathrm{H}, 3.6991663313,-1.5554792977,2.6600768045$ $\mathrm{H}, 4.4585456574,-1.5139703452,1.0604373083$ $\mathrm{H}, 5.1341502961,-0.5533024101,2.386771199$ $\mathrm{H}, 4.2320858126,1.066215059,-0.7118878875$ $\mathrm{H}, 4.8635687674,2.0826479524,0.593913191$ $\mathrm{H}, 3.2953887681,2.4563721138,-0.1404203322$ C, $-0.4470138315,-2.3605452013,0.8676095999$ $\mathrm{H},-1.1496471699,-2.8674601584,0.1962110077$ $\mathrm{H}, 0.4838603003,-2.9409448539,0.9062975442$ $\mathrm{H}, 1.4775899409,-1.1839563297,-1.9828447194$ C, $-1.0220156684,-2.1006666452,2.2138223284$ $C_{,}, 0.2716867407,-1.9790391367,3.3244929712$ C, $-2.5209755417,-1.9536483276,2.2748426983$ $\mathrm{H}, 0.8076859148,-2.1040004601,3.2969295722$ $\mathrm{H},-3.0097668575,-2.8953969745,1.9941296609$ $\mathrm{H},-2.8646671073,-1.6767072271,3.2754265572$ $\mathrm{H},-2.8654640814,-1.1935241449,1.5643655074$ $\mathrm{H},-0.7192998218,-1.7672917823,4.2916359953$

\section{$\mathrm{R}=i-\mathrm{Pr}, \mathrm{H}=-753.289732$}

Ti, $-0.2514082544,0.4667033464,0.0409378264$ Si, $-1.6676997734,-0.437214828,-2.9034702457$ $\mathrm{Si}, 2.9891854564,-0.5848973108,-0.5303145327$ $\mathrm{N},-1.608034523,-0.0379031608,-1.1762055263$ $0,1.3886221533,-0.0347765568,-0.488921673$ C, $3.9604178049,0.5450370409,-1.6879971681$ C, $2.985558035,-2.3588854525,-1.1751961827$ C, $3.7038069605,-0.5177306552,1.2161737303$ $0,-0.7434837356,0.0552470694,1.7124929976$ $\mathrm{Si},-1.0730672286,-0.6634914289,3.209796563$ C, $-2.9462628362,-0.6942482634,3.4395850547$ $C_{,}-0.37898778,-2.4191080113,3.1974157875$ C, $-0.2514699354,0.3745362803,4.5536590212$ $C,-0.2234107424,0.4230160539,-3.7633132587$ $\mathrm{H},-0.3269384179,1.511185779,-3.7058303867$ $\mathrm{H}, 0.7255895698,0.1474867238,-3.2937276464$ $\mathrm{H},-0.1755102545,0.1411098705,-4.8205847317$ C, $-1.5431421766,-2.3112870821,-3.1568217891$ $\mathrm{H},-0.6047559612,-2.692502947,-2.7414029898$ $\mathrm{H},-2.3667296774,-2.8310552998,-2.6556595369$ $\mathrm{H},-1.5770675689,-2.5742819554,-4.2199997588$ $C,-3.3188995943,0.1772772378,-3.6023777676$ $\mathrm{H},-3.4031278067,-0.051594285,-4.6704432703$ $\mathrm{H},-4.1663377867,-0.2977585684,-3.0956963557$ $\mathrm{H},-3.4162105613,1.2606011755,-3.479596842$ $\mathrm{H}, 4.002842233,-2.7637943416,-1.2111756115$ $\mathrm{H}, 2.3858006959,-3.0077270695,-0.5290630712$ $\mathrm{H}, 2.5675574102,-2.40634617,-2.1855834686$ $\mathrm{H}, 3.9335855317,1.5807701515,-1.3352603497$ $\mathrm{H}, 5.0093546322,0.2348620751,-1.7488035456$ $\mathrm{H}, 3.5421924278,0.5219149459,-2.6991150325$ $\mathrm{H}, 3.6840833965,0.5035471293,1.6093842573$ $\mathrm{H}, 3.1312766732,-1.1552290197,1.897154788$ $\mathrm{H}, 4.7434137576,-0.8632489184,1.2249872762$ $\mathrm{H},-0.4409106902,-0.048052114,5.546434824$ $\mathrm{H},-0.6371990555,1.3987164979,4.5428780217$ 
$\mathrm{H}, 0.8320900062,0.4186866827,4.4059384766$

$\mathrm{H},-3.3601442837,0.3185166365,3.4072187116$

$\mathrm{H},-3.4268853867,-1.2838267393,2.6522193096$

$\mathrm{H},-3.2152461586,-1.1389445497,4.4039677585$

$\mathrm{H},-0.8310677072,-3.0111669342,2.3952089948$

$\mathrm{H},-0.5801884495,-2.9266416519,4.1472259774$

$\mathrm{H}, 0.704643505,-2.4099548499,3.0437030755$

C, $-0.2217343267,2.5606536609,-0.0210156819$ $\mathrm{H}, 0.0583453274,2.775675199,-1.0655517786$

$\mathrm{H},-2.5087719819,-0.1656159905,-0.7155211461$

C, $0.8641261808,3.1059856813,0.9092541041$

$\mathrm{H}, 0.9374922608,4.2028559027,0.8424635012$

$\mathrm{H}, 0.6503950957,2.8652494732,1.9580865371$

$\mathrm{H}, 1.8539018111,2.700273086,0.6724929853$

C, $-1.5870681581,3.1843630401,0.2673375538$

$\mathrm{H},-1.9409187073,2.9279129859,1.2739725052$

$\mathrm{H},-1.5465706366,4.2840034441,0.2154117217$

$\mathrm{H},-2.3473262772,2.8492508147,-0.4458826965$

\section{$\mathrm{R}=t-\mathrm{Bu}, \mathrm{H}=-792.561483$}

$\begin{array}{lrrr}\text { Ti } & 0.05926 & -0.22244 & 0.44427 \\ \text { Si } & -2.76342 & -1.69144 & -0.80278 \\ \text { Si } & -0.45671 & 3.03849 & -0.51403 \\ \text { N } & -1.12058 & -1.58226 & -0.14134 \\ \text { O } & -0.49633 & 1.43099 & 0.01996 \\ \text { C } & -1.68911 & 4.01981 & 0.52387 \\ \text { C } & -0.94612 & 3.06535 & -2.33723 \\ \text { C } & 1.29083 & 3.71778 & -0.29147 \\ \text { O } & 1.7452 & -0.65002 & 0.00791 \\ \text { Si } & 3.17369 & -1.04355 & -0.81004 \\ \text { C } & 3.32986 & -2.92483 & -0.84511 \\ \text { C } & 3.07214 & -0.35831 & -2.56646 \\ \text { C } & 4.61562 & -0.27036 & 0.12897 \\ \text { C } & -3.72475 & -0.14607 & -0.30421 \\ \text { H } & -3.86873 & -0.10215 & 0.7795 \\ \text { H } & -3.18916 & 0.75707 & -0.60996 \\ \mathrm{H} & -4.71145 & -0.13816 & -0.77963 \\ \mathrm{C} & -2.70136 & -1.82495 & -2.69347 \\ \mathrm{H} & -2.2182 & -0.94348 & -3.12738\end{array}$

$\begin{array}{llrl}\mathrm{H} & -2.13357 & -2.70731 & -3.00796 \\ \mathrm{H} & -3.70763 & -1.90529 & -3.11983 \\ \mathrm{C} & -3.5953 & -3.24421 & -0.1031 \\ \mathrm{H} & -4.61191 & -3.35589 & -0.49598 \\ \mathrm{H} & -3.03731 & -4.14891 & -0.36882 \\ \mathrm{H} & -3.65729 & -3.19525 & 0.98863 \\ \mathrm{H} & -0.94195 & 4.08888 & -2.72785 \\ \mathrm{H} & -0.24822 & 2.47228 & -2.93676 \\ \mathrm{H} & -1.95026 & 2.65415 & -2.4803 \\ \mathrm{H} & -1.41489 & 3.99347 & 1.58314 \\ \mathrm{H} & -1.71974 & 5.06846 & 0.20846 \\ \mathrm{H} & -2.69776 & 3.60679 & 0.42522 \\ \mathrm{H} & 1.59115 & 3.69554 & 0.76076 \\ \mathrm{H} & 2.01494 & 3.12899 & -0.86325 \\ \mathrm{H} & 1.35011 & 4.75539 & -0.63804 \\ \mathrm{H} & 5.56851 & -0.49087 & -0.3644 \\ \mathrm{H} & 4.66722 & -0.65637 & 1.15183 \\ \mathrm{H} & 4.50613 & 0.81732 & 0.18281 \\ \mathrm{H} & 3.33677 & -3.33526 & 0.16954 \\ \mathrm{H} & 2.49439 & -3.37679 & -1.38959 \\ \mathrm{H} & 4.25791 & -3.23163 & -1.33982 \\ \mathrm{H} & 2.19958 & -0.76152 & -3.09053 \\ \mathrm{H} & 3.96531 & -0.6246 & -3.1423 \\ \mathrm{H} & 2.98985 & 0.73313 & -2.55886 \\ \mathrm{C} & 0.02922 & -0.32321 & 2.54895 \\ \mathrm{H} & -0.63389 & -2.47821 & -0.19229 \\ \mathrm{C} & 1.0348 & 0.71265 & 3.06441 \\ \mathrm{H} & 1.06377 & 0.70711 & 4.1673 \\ \mathrm{H} & 2.05249 & 0.50858 & 2.71264 \\ \mathrm{H} & 0.76998 & 1.73087 & 2.7562 \\ \mathrm{C} & 0.44142 & -1.72684 & 3.00022 \\ \mathrm{H} & 1.4357 & -2.00201 & 2.62995 \\ \mathrm{H} & 0.47763 & -1.78479 & 4.10156 \\ \mathrm{H} & -0.26981 & -2.48779 & 2.65951 \\ \mathrm{C} & -1.37545 & 0.00535 & 3.05977 \\ \mathrm{H} & -1.39786 & -0.00015 & 4.16307 \\ \mathrm{H} & -1.71075 & 0.99687 & 2.73473 \\ \mathrm{H} & -2.11218 & -0.72881 & 2.71615 \\ & & & \end{array}$




\section{Cartesian coordinates for the Wolczanski series Ti(OSit-Bu $)_{2}(\mathrm{NHSi} t-\mathrm{Bu})(\mathrm{R})$ and en- thalpies (au) at BP86}

\author{
Fragment Ti(OSit-Bu $)_{2}(\mathrm{NHSi} t-\mathrm{Bu})^{\bullet}, \mathrm{H}=-635.097500$
}

Ti, $0.0537523039,-0.3278088425,-0.0430610227$ $\mathrm{Si},-3.0614672119,-1.7770536704,0.0056805249$ Si, $3.443056313,-1.2251030331,0.0075777538$ $\mathrm{N},-1.2763107254,-1.6966070224,0.0210835299$ $0,1.7660720455,-0.9038179402,-0.0293639333$ C, $3.6693232266,-3.0861251729,0.2983132235$ C, $4.2064686493,-0.2213250531,1.4243963596$ C, $4.1751020236,-0.7097863525,-1.6644249264$ $0,-0.4484761788,1.4130670369,-0.0395365203$ $\mathrm{Si},-0.4052690238,3.1198648605,0.0056363413$ C, $-1.6769999921,3.7656910919,-1.2443787006$ C, $-0.8478922298,3.6635326943,1.7683999337$ C, $1.3426856673,3.6946995892,-0.4599292217$ $C,-3.7041531444,-2.2514665778,1.7350001545$ $\mathrm{H},-3.3021230274,-3.2285937919,2.0538905006$ $\mathrm{H},-3.3969020714,-1.5013842719,2.4832247248$ $\mathrm{H},-4.8061756947,-2.3197983573,1.7500329695$ $\mathrm{C},-3.7229992459,-0.0715888596,-0.49634955$ $\mathrm{H},-3.3600064337,0.7120876216,0.1887726807$ $\mathrm{H},-3.3931527444,0.1904830543,-1.5155321927$ $\mathrm{H},-4.8266070412,-0.0595342446,-0.4790205434$ C, $-3.6128238746,-3.0964434221,-1.2537805782$ $\mathrm{H},-4.713542616,-3.1747828121,-1.2914760208$ $\mathrm{H},-3.253895668,-2.8467703724,-2.2663971615$ $\mathrm{H},-3.2180054961,-4.093390557,-0.9910932498$ $\mathrm{H}, 5.2910027054,-0.4102780915,1.5072408921$ $\mathrm{H}, 4.0621944557,0.8599748389,1.2615435829$ $\mathrm{H}, 3.7400532128,-0.4864728047,2.3880664596$ $\mathrm{H}, 3.2009318368,-3.6694766045,-0.5122653405$ $\mathrm{H}, 4.7394147657,-3.3550548668,0.3351229202$ $\mathrm{H}, 3.2091576638,-3.3948474674,1.2520406369$ $\mathrm{H}, 3.6976417815,-1.2660970326,-2.4884375787$ $\mathrm{H}, 4.0205523196,0.3670881742,-1.845523035$ $\mathrm{H}, 5.2603709503,-0.9089148749,-1.7025495717$ $\mathrm{H}, 1.415450599,4.7963219881,-0.4556138945$ $\mathrm{H}, 1.6139854031,3.340232999,-1.4686758934$ $\mathrm{H}, 2.0879197432,3.3024255187,0.2524728522$ $\mathrm{H},-1.4279796658,3.431725175,-2.265617067$ $\mathrm{H},-2.687616162,3.3963728015,-1.0033451283$ $\mathrm{H},-1.7074391789,4.8691996328,-1.2450147626$ $\mathrm{H},-1.8510240419,3.3017618636,2.049740592$ $\mathrm{H},-0.8451898554,4.7638512494,1.8575329794$ $\mathrm{H},-0.1245485674,3.2607976986,2.4972348336$ $\mathrm{H},-0.8543572749,-2.6339173696,0.096601388$

\section{$\mathrm{R}=\mathrm{Ph}, \mathrm{H}=-866.672062$}

Ti, $0.1085135537,-0.0890031208,-0.1299415401$ Si, $-2.2890642433,-2.3097967227,-1.0789668286$ $\mathrm{Si}, 3.3265153797,-1.2642260343,0.3692707124$ $\mathrm{N},-0.6906339458,-1.5010775053,-1.1105844968$ $0,1.8274095301,-0.4472544738,0.2650111895$ C, $3.6423291223,-2.1356846897,-1.2862450841$ C, $3.2060832964,-2.5201682519,1.7850055155$ C, $4.6620722776,0.0352895616,0.7154352843$ $0,-0.9297153636,0.3980782555,1.258653031$ $\mathrm{Si},-1.2604946541,1.3300316911,2.6594247954$ $C_{,}-2.8038542027,2.3684045574,2.2996701499$ C, $-1.5624783553,0.1173315741,4.086337646$ C, $0.2385050366,2.4366603538,3.0115913547$
$C,-2.3072654386,-3.6722840471,0.2509391389$ $\mathrm{H},-1.5370209532,-4.435813448,0.0470825989$ $\mathrm{H},-2.100629714,-3.2407529079,1.245046467$ $\mathrm{H},-3.2861659844,-4.1816657041,0.2952124773$ $C,-3.6043576547,-1.0027578471,-0.6878275303$ $\mathrm{H},-3.3139173742,-0.432224318,0.2103133534$ $\mathrm{H},-3.7135618771,-0.2896802449,-1.5221228426$ $\mathrm{H},-4.5871678855,-1.4690478546,-0.5016600888$ C, $-2.5841054868,-3.089366855,-2.7923584911$ $\mathrm{H},-3.5579889775,-3.6084907572,-2.8248162225$ $\mathrm{H},-2.5822509777,-2.3223691139,-3.5851509315$ $\mathrm{H},-1.8053459192,-3.8330621336,-3.036400529$ $\mathrm{H}, 4.1482485104,-3.0843760766,1.8984104423$ $\mathrm{H}, 2.994373476,-2.0147368845,2.7421584058$ $\mathrm{H}, 2.3959545345,-3.2444174855,1.5952872222$ $\mathrm{H}, 3.6271588267,-1.4107098638,-2.1174939443$ $\mathrm{H}, 4.6256159379,-2.6377418727,-1.2898825127$ $\mathrm{H}, 2.8739800235,-2.9028097529,-1.4834607519$ $\mathrm{H}, 4.7037700041,0.780252166,-0.0966036679$ $\mathrm{H}, 4.4569278494,0.5681484455,1.6589843899$ $\mathrm{H}, 5.6580733981,-0.4335915806,0.7991251248$ $\mathrm{H}, 0.0677307044,3.0663807963,3.902242046$ $\mathrm{H}, 0.4375297437,3.1031429218,2.1554958629$ $\mathrm{H}, 1.141253563,1.8289124014,3.1916804346$ $\mathrm{H},-2.6299900665,3.0314579057,1.4358247174$ $\mathrm{H},-3.669241791,1.7252271548,2.0677864483$ $\mathrm{H},-3.0698542061,2.9970157995,3.1674552647$ $\mathrm{H},-2.4046313826,-0.5570515621,3.8574275927$ $\mathrm{H},-1.8016088396,0.6544874074,5.0207669612$ $\mathrm{H},-0.6688471229,-0.5028902424,4.2691218366$ $\mathrm{H},-0.0187486045,-1.9802584973,-1.7266610949$ C, $0.1796335922,1.547552728,-1.447165024$ C, $1.1277763329,2.58017224,-1.2271517573$ C, $1.1593438869,3.7363423147,-2.0274732309$ C, $0.2370298092,3.8881891372,-3.0763197367$ C, $-0.710224738,2.8799137429,-3.3208467138$ C, $-0.734213389,1.7269321686,-2.5164268535$ $\mathrm{H}, 1.8652479088,2.4803541042,-0.4195764109$ $\mathrm{H}, 1.9054476342,4.5169097928,-1.8359551666$ $\mathrm{H}, 0.260086377,4.7864887284,-3.7035615385$ $\mathrm{H},-1.4281666591,2.9912144507,-4.1422540158$ $\mathrm{H},-1.475709336,0.9474339637,-2.7295198459$

$$
\mathrm{R}=\text { Vinyl, } \mathrm{H}=-713.065723
$$

Ti, $-0.051083118,-0.1454259492,0.6662607811$ Si, $2.4617254764,-2.1859524614,-0.3551272025$ $\mathrm{Si},-3.2590886314,-0.6837098148,-0.5167046674$ $\mathrm{N}, 0.8217018845,-1.7742446825,0.2360326549$ $0,-1.7907194681,-0.1849323872,0.2052392723$ C, $-3.4813905097,-2.5394945466,-0.1892870136$ C, $-3.1446977787,-0.3342389943,-2.377184241$ C, $-4.6569972048,0.3181922731,0.2802172189$ $0,0.9095169063,1.2641302103,0.0820631391$ $\mathrm{Si}, 1.0062904855,2.9188851799,-0.3593955857$ C, $2.5364486994,3.6393573698,0.4955865719$ C, $1.1782177798,3.0024307766,-2.2466343583$ C, $-0.5755969573,3.7878601362,0.2206546356$ C, $2.5272379756,-2.0253367964,-2.250843114$ $\mathrm{H}, 1.8084777858,-2.7112170993,-2.7310800433$ $\mathrm{H}, 2.2691553184,-0.9972652662,-2.5565496009$ $\mathrm{H}, 3.532882968,-2.257037069,-2.6437752747$ 
C, $3.6959145078,-0.9874824739,0.4425700983$ $\mathrm{H}, 3.3789883501,0.0539551513,0.2664700604$ $\mathrm{H}, 3.7477161169,-1.1486814239,1.5324737694$ $\mathrm{H}, 4.709708658,-1.1176472333,0.0261445912$ C, $2.8374517503,-3.9829075028,0.1519308183$ $\mathrm{H}, 3.8439750256,-4.2825016809,-0.1885336448$ $\mathrm{H}, 2.8015778641,-4.0999484856,1.2481743151$ $\mathrm{H}, 2.1141905233,-4.6895781593,-0.2911652202$ $\mathrm{H},-4.0647296849,-0.6516895869,-2.8982890438$ $\mathrm{H},-3.0019897709,0.7429478219,-2.56598513$ $\mathrm{H},-2.2947342234,-0.8755066169,-2.8260302291$ $\mathrm{H},-3.4738402193,-2.7507849138,0.8932447678$ $\mathrm{H},-4.4392899558,-2.9032922181,-0.6002640026$ $\mathrm{H},-2.6714222707,-3.1225800462,-0.6598225137$ $\mathrm{H},-4.7050025891,0.1344393737,1.3665396401$ $\mathrm{H},-4.5036959197,1.3991007296,0.1243963481$ $\mathrm{H},-5.6350762804,0.046819221,-0.1539586085$ $\mathrm{H},-0.5586797531,4.8618432641,-0.0348186832$ $\mathrm{H},-0.690194982,3.6968624568,1.3139034934$ $\mathrm{H},-1.4640009505,3.3378144619,-0.2532636576$ $\mathrm{H}, 2.4510602194,3.5470083909,1.5911989088$ $\mathrm{H}, 3.450366753,3.1097851189,0.1785678758$ $\mathrm{H}, 2.6606006786,4.7085805638,0.2503933379$ $\mathrm{H}, 2.0902337233,2.4816135831,-2.5833429455$ $\mathrm{H}, 1.2382140614,4.0493231269,-2.5921554952$ $\mathrm{H}, 0.3127602169,2.5307768598,-2.7416337247$ $\mathrm{H}, 0.2115811125,-2.5981601918,0.3254911534$ C, $-0.0257036754,-0.0613988729,2.7503444274$ C, $-1.1192793183,0.1118407967,3.5284090705$ $\mathrm{H}, 0.9461243835,-0.1458667843,3.2711848693$ $\mathrm{H},-1.0720563418,0.1699695608,4.6280113416$ $\mathrm{H},-2.125979158,0.2043645207,3.0986879803$

\section{$\mathrm{R}=\mathrm{Me}, \mathrm{H}=-674.998283$}

$\mathrm{Ti},-0.111099871,0.0904789332,0.6845167003$ $\mathrm{Si},-0.0723181387,3.4104142408,-0.0311528511$ $\mathrm{Si}, 2.6727139332,-1.1421081299,-0.8758582936$ $\mathrm{N},-0.8058560883,1.8266554583,0.3549513813$ $0,1.4876412248,-0.1749083483,-0.109648734$ C, $4.3533596737,-0.31393302,-0.587195516$ C, $2.264904409,-1.2260461948,-2.7266275421$ C, $2.6277967653,-2.869992364,-0.0947212001$ $0,-1.3782099218,-1.1507271967,0.4070891519$ $\mathrm{Si},-2.6096902292,-2.3002232909,0.1212416935$ C, $-4.2072431629,-1.6447010846,0.9051885289$ $C_{,}-2.8052984212,-2.4922987856,-1.7553982347$ $C,-2.083549554,-3.9348406464,0.9237033494$ C, $1.7391080951,3.3708347796,0.5288503474$ $\mathrm{H}, 1.8101634721,3.2786821471,1.6255567184$ $\mathrm{H}, 2.2650544003,2.5117550634,0.0804148826$ $\mathrm{H}, 2.2649649977,4.2933579502,0.2281568651$ C, $-0.1717858762,3.7422873618,-1.9040912625$ $\mathrm{H}, 0.3685125906,2.9607473577,-2.4643928974$ $\mathrm{H},-1.2191190703,3.742892605,-2.2516630807$ $\mathrm{H}, 0.2712524472,4.71951086,-2.164654468$ C, $-1.0395019863,4.7605537265,0.9016522211$ $\mathrm{H},-0.6224235301,5.7614803159,0.6944154893$ $\mathrm{H},-2.1017641242,4.7756748754,0.6015292482$ $\mathrm{H},-0.9967077306,4.5918760407,1.9906445534$ $\mathrm{H}, 3.01784793,-1.8229426932,-3.2702454096$ $\mathrm{H}, 1.278790976,-1.691530869,-2.891582731$ $\mathrm{H}, 2.2441577841,-0.2176355165,-3.1722617839$ $\mathrm{H}, 4.5666706101,-0.2286420234,0.4912770884$ $\mathrm{H}, 5.1668377729,-0.8987034194,-1.0506443736$
$\mathrm{H}, 4.3754039801,0.6997784715,-1.0206377787$ $\mathrm{H}, 2.8469149098,-2.8153071562,0.9846693258$ $\mathrm{H}, 1.6317740761,-3.3277284594,-0.2175072743$ $\mathrm{H}, 3.3708787586,-3.5401989088,-0.5606759655$ $\mathrm{H},-2.8540177973,-4.7123280104,0.7804882864$ $\mathrm{H},-1.9264067801,-3.8053896068,2.0073801066$ $\mathrm{H},-1.1423791966,-4.3052627394,0.4843109546$ $\mathrm{H},-4.0741237708,-1.4837963713,1.987987175$ $\mathrm{H},-4.5067485651,-0.685453146,0.4505508941$ $\mathrm{H},-5.037991863,-2.3582689146,0.7665696146$ $\mathrm{H},-3.0601550248,-1.5273968434,-2.2246676917$ $\mathrm{H},-3.6071226894,-3.211222671,-1.9977954383$ $\mathrm{H},-1.8714440497,-2.8583652998,-2.213633029$ C, $0.2701883345,0.0284295572,2.7486178337$ $\mathrm{H}, 1.0231968995,0.7889845078,3.0240994409$ $\mathrm{H},-0.652760306,0.225729795,3.3236496247$ $\mathrm{H}, 0.656425688,-0.96966034,3.0295724205$ $\mathrm{H},-1.8336910155,1.8624031616,0.3864399807$

\section{$\mathrm{R}=\mathrm{Et}, \mathrm{H}=-714.276950$}

Ti, $0.0277041331,-0.3305504421,0.5524484478$ Si, $-2.9748615225,-1.6802950175,-0.3096974115$ $\mathrm{Si},-0.441916367,2.9561937871,-0.4241838034$ $\mathrm{N},-1.213181697,-1.656942663,-0.0141645162$ $0,-0.504348209,1.3463205853,0.1457364864$ C, $-1.6251957876,3.9861660431,0.6408802621$ C, $-0.9921788302,2.9563304145,-2.2400690624$ C, $1.3381681679,3.5943559282,-0.2655828842$ $0,1.700612152,-0.8135966674,0.1017598541$ $\mathrm{Si}, 3.2449542132,-1.1831127308,-0.5290675691$ C, $3.5856899288,-3.0149051077,-0.1748165787$ C, $3.2174687026,-0.8468378385,-2.3958552366$ C, $4.515135711,-0.0756568979,0.3393759715$ $\mathrm{C},-3.7465943472,-0.2266220058,0.634306268$ $\mathrm{H},-3.6430965418,-0.3650968311,1.723724161$ $\mathrm{H},-3.2484692111,0.71931794,0.3639575703$ $\mathrm{H},-4.8212679487,-0.1300280112,0.4021775413$ $C,-3.3360630509,-1.5158973055,-2.1730829598$ $\mathrm{H},-2.9359483516,-0.5647521803,-2.5630199732$ $\mathrm{H},-2.8658776393,-2.3365541913,-2.7418426442$ $\mathrm{H},-4.4208984519,-1.5415877661,-2.3779846128$ $C,-3.6701763076,-3.3380872278,0.3210085963$ $\mathrm{H},-4.7587705076,-3.4018889078,0.1482343834$ $\mathrm{H},-3.2018778402,-4.1939605449,-0.1958270322$ $\mathrm{H},-3.4885304077,-3.4542896953,1.4026273144$ $\mathrm{H},-0.9907440072,3.9799078391,-2.6537733035$ $\mathrm{H},-0.3171422098,2.3396112472,-2.8569404354$ $\mathrm{H},-2.0125540485,2.5505301098,-2.3416849306$ $\mathrm{H},-1.3277976313,3.9531322345,1.702279791$ $\mathrm{H},-1.6284191167,5.0425964793,0.3203142997$ $\mathrm{H},-2.6573881454,3.6052458999,0.565285589$ $\mathrm{H}, 1.6717886766,3.5779879277,0.7854569252$ $\mathrm{H}, 2.0321351445,2.9713910177,-0.8546216392$ $\mathrm{H}, 1.4192261501,4.6325219947,-0.6324220589$ $\mathrm{H}, 5.5365042714,-0.2833136543,-0.0245961085$ $\mathrm{H}, 4.5019559999,-0.2428136715,1.4293301743$ $\mathrm{H}, 4.2987132331,0.9899682303,0.1554541424$ $\mathrm{H}, 3.5334746479,-3.2193934898,0.9076356319$ $\mathrm{H}, 2.8469277575,-3.6592489891,-0.6805762052$ $\mathrm{H}, 4.5893195269,-3.3079482942,-0.5292024037$ $\mathrm{H}, 2.4572364909,-1.4683785157,-2.8981380843$ $\mathrm{H}, 4.1970564087,-1.0716856516,-2.8527278315$ $\mathrm{H}, 2.982712193,0.2112036667,-2.600841492$ C, $-0.0254876046,-0.4326397764,2.6544225556$ 
$\mathrm{H},-1.0796108349,-0.2560143128,2.9487099246$ $\mathrm{H}, 0.2088645995,-1.4807268516,2.9293751354$ $\mathrm{H},-0.7643447217,-2.5591153067,-0.2236064531$ C, $0.9198224602,0.5513301493,3.3645430805$ $\mathrm{H}, 0.8616274596,0.473864365,4.4688411942$ $\mathrm{H}, 1.9744086498,0.3748623049,3.0859066367$ $\mathrm{H}, 0.6854530269,1.5993498344,3.1043127332$

\section{$\mathrm{R}=\mathrm{Pr}, \mathrm{H}=-753.561165$}

Ti, $0.0092376613,-0.2899091824,0.393660398$ $\mathrm{Si},-3.0209760371,-1.7480382898,-0.1608153612$ $\mathrm{Si},-0.4680710604,2.8648617474,-0.9325633379$ $\mathrm{N},-1.2508957439,-1.6780354496,0.0730639855$ $0,-0.5475394671,1.3161228031,-0.212820285$ C, $-1.6899326171,3.9837980893,-0.0105504153$ C, $-0.9542654028,2.6860991399,-2.7577498126$ C, $1.303432358,3.5252470337,-0.7703977754$ $0,1.6610846679,-0.8316883186,-0.0689706527$ $\mathrm{Si}, 3.1259954557,-1.3401875896,-0.7868281942$ C, $3.1360663272,-3.2371958699,-0.7967326321$ C, $3.1917963957,-0.6544036289,-2.5542580258$ C, $4.5516852724,-0.6594778017,0.2603617858$ $C,-3.7663130491,-0.1322195578,0.4968727446$ $\mathrm{H},-3.6284132744,-0.0503119648,1.5882050012$ $\mathrm{H},-3.2806996702,0.7394397877,0.0278867883$ $\mathrm{H},-4.8480868345,-0.0825711872,0.2836553009$ $C,-3.4347782307,-1.9544497407,-2.0092826468$ $\mathrm{H},-3.040195044,-1.1054857616,-2.5927880706$ $\mathrm{H},-2.9886845785,-2.8783542306,-2.4161421575$ $\mathrm{H},-4.5254701758,-2.0056523886,-2.1746291255$ $C,-3.6990094818,-3.2413851288,0.8083565858$ $\mathrm{H},-4.7943375107,-3.323401441,0.6961801579$ $\mathrm{H},-3.2573927869,-4.1886127105,0.4525880902$ $\mathrm{H},-3.4726122657,-3.144641839,1.8835008661$ $\mathrm{H},-0.9349908948,3.6636386188,-3.2704979797$ $\mathrm{H},-0.2598655097,2.0112945174,-3.2859936874$ $\mathrm{H},-1.9717740115,2.2720339317,-2.8546451433$ $\mathrm{H},-1.4295706542,4.0493968953,1.0591179837$ $\mathrm{H},-1.6822233126,5.0063155339,-0.4267782749$ $\mathrm{H},-2.7186932479,3.5938746758,-0.086567559$ $\mathrm{H}, 1.599560981,3.6009896219,0.2893029017$ $\mathrm{H}, 2.0201624425,2.8583046189,-1.278406417$ $\mathrm{H}, 1.3941991339,4.5284589074,-1.2224040743$ $\mathrm{H}, 5.5287308874,-0.9721532508,-0.1475515754$ $\mathrm{H}, 4.4832859665,-1.0250737376,1.2984873193$ $\mathrm{H}, 4.5300528975,0.4428556652,0.2848634954$ $\mathrm{H}, 3.0624356877,-3.6351292933,0.229325719$ $\mathrm{H}, 2.2879305912,-3.632940639,-1.380731279$ $\mathrm{H}, 4.0664141746,-3.6263546847,-1.2459252761$ $\mathrm{H}, 2.3427294134,-1.0248398123,-3.1529500993$ $\mathrm{H}, 4.1244556909,-0.9560570929,-3.0621789567$ $\mathrm{H}, 3.151306825,0.4478286938,-2.5481880754$ C, $0.0578148105,-0.1067341171,2.4912134869$ $\mathrm{H},-0.9841577991,0.0957884456,2.8154829764$ $\mathrm{H}, 0.3244208331,-1.1075060046,2.8910897082$ $\mathrm{H},-0.8075746932,-2.6041472098,-0.0014247538$ C, $1.0174001731,0.9686829723,3.0310426514$ $\mathrm{H}, 2.0452820167,0.7677938113,2.6704125657$ $\mathrm{H}, 0.7390715608,1.9578598882,2.6170999943$ C, $1.0403180524,1.0639085235,4.5705764441$ $\mathrm{H}, 1.7389688631,1.8442627563,4.9239027337$ $\mathrm{H}, 0.0370314777,1.3040873657,4.9655052811$ $\mathrm{H}, 1.3508182122,0.1036621611,5.0197286306$

$\mathrm{R}=c-\mathrm{Pr}, \mathrm{H}=-752.342904$
$\mathrm{Ti}, 0.0531088111,0.0721543702,0.5628948017$ $\mathrm{Si},-1.2738823154,3.1567041718,-0.0788351394$ Si, $3.2676999142,-0.7788719706,-0.3999055985$ $\mathrm{N},-0.0515441677,1.9684677863,0.4560967714$ $0,1.7572059875,-0.4777979951,0.3416487348$ C, $4.4875668623,0.537459079,0.2155396995$ C, $3.0430557396,-0.6628361616,-2.2800941848$ C, $3.8317097215,-2.5147003068,0.1133967982$ $0,-1.2107145894,-0.72053808,-0.4506719996$ $\mathrm{Si},-2.0695678077,-1.9972966242,-1.1973272569$ $C,-3.6589319222,-2.2823078662,-0.20454452$ $C_{,}-2.4622107023,-1.4605062026,-2.9741839353$ C, $-0.9818771288,-3.551986749,-1.1886919675$ C, $-2.9791174603,2.3345682795,0.0343589289$ $\mathrm{H},-2.9719468309,1.3733908369,-0.5057241205$ $\mathrm{H},-3.2480697337,2.1325160841,1.0848389804$ $\mathrm{H},-3.7627134521,2.9757339106,-0.4045626108$ $\mathrm{C},-1.1768955987,4.6763246393,1.0659440431$ $\mathrm{H},-1.3809704895,4.394061938,2.1124268189$ $\mathrm{H},-0.1783809038,5.1459839392,1.0290463977$ $\mathrm{H},-1.9142981285,5.4423087013,0.7685478773$ C, $-0.9277899077,3.6970418455,-1.8726880302$ $\mathrm{H},-1.6715061399,4.4342449936,-2.2235070389$ $\mathrm{H}, 0.0712610527,4.1574741282,-1.9622475513$ $\mathrm{H},-0.9604003576,2.8282317008,-2.5517739405$ $\mathrm{H}, 4.0014042349,-0.8168890577,-2.8061027191$ $\mathrm{H}, 2.3324118003,-1.4261156699,-2.6391174279$ $\mathrm{H}, 2.6524903477,0.328520072,-2.5656649982$ $\mathrm{H}, 4.588803394,0.498086757,1.313077722$ $\mathrm{H}, 5.4890339855,0.3856674588,-0.2235992413$ $\mathrm{H}, 4.1480614671,1.5500735253,-0.0607151397$ $\mathrm{H}, 3.9335795559,-2.5873609958,1.2090613923$ $\mathrm{H}, 3.1052634046,-3.2776239689,-0.212760713$ $\mathrm{H}, 4.8092440803,-2.7617787089,-0.3362151263$ $\mathrm{H},-1.493480511,-4.3945468114,-1.6859500365$ $\mathrm{H},-0.7452207127,-3.8590768377,-0.1562203688$ $\mathrm{H},-0.0319555008,-3.371080115,-1.7191089988$ $\mathrm{H},-3.4204572319,-2.5463276243,0.8392642916$ $\mathrm{H},-4.2851512188,-1.3747374133,-0.1918386442$ $\mathrm{H},-4.2566856247,-3.1031020138,-0.6379209183$ $\mathrm{H},-3.0853255022,-0.5506010991,-2.9824002086$ $\mathrm{H},-3.0104184172,-2.2522082358,-3.5140419096$ $\mathrm{H},-1.537118602,-1.2446722821,-3.5345536368$ $\mathrm{H}, 0.8217580073,2.4160784502,0.7682554746$ C, $-0.3932087964,-0.3938029491,2.5345930984$ C, $0.0591367332,-1.7225215968,3.1484958021$ C, $0.674224753,-0.4279919307,3.6286226608$ $\mathrm{H},-1.379792089,-0.0476322803,2.8807735982$ $\mathrm{H}, 0.6833461637,-2.3939785795,2.5470018068$ $\mathrm{H},-0.6324512479,-2.2466907432,3.8190181125$ $\mathrm{H}, 0.4025386988,-0.0667608452,4.6276942789$ $\mathrm{H}, 1.7141598114,-0.2211098221,3.3513032492$

\section{$\mathrm{R}=c-\mathrm{Pe}, \mathrm{H}=-830.939045$}

Ti, $-0.1120643989,0.1904103315,-0.0568822593$ $\mathrm{Si}, 3.2416837691,-0.5687564818,-0.3066048548$ Si, $-1.1600869035,-1.4384823995,2.7623190921$ $\mathrm{N}, 1.6406464108,0.0550956067,-0.799108306$ $0,-0.2702976723,-0.6403333061,1.5363305446$ C, $-0.0231153185,-1.6039812971,4.2704991719$ $C_{,}-1.6774331536,-3.1442999105,2.1125945204$ $C_{1},-2.6819701345,-0.3835968005,3.1734075237$ $0,-1.3901438683,-0.1664722107,-1.2814550833$ Si, $-2.2240315439,-0.7461137432,-2.653729815$ C, $-1.4767061281,0.0720109855,-4.1950433022$ 
$C,-2.009830096,-2.6295407639,-2.7221619809$ $C,-4.0491365849,-0.2697205596,-2.4600618605$ C, $3.0121712856,-1.7369272272,1.1688886533$ $\mathrm{H}, 2.4922210969,-1.2266171489,1.9957795664$ $\mathrm{H}, 2.4111317214,-2.6160955911,0.8833452543$ $\mathrm{H}, 3.9891275696,-2.0922902908,1.5397814573$ C, $4.0089033132,-1.5033541391,-1.7801611913$ $\mathrm{H}, 3.3798519814,-2.3605020831,-2.0745527958$ $\mathrm{H}, 4.1211045068,-0.8453633857,-2.6597397197$ $\mathrm{H}, 5.0117881405,-1.8889387859,-1.5258765747$ C, $4.3866743941,0.8731361649,0.183590748$ $\mathrm{H}, 5.3857811248,0.5090690759,0.4814698776$ $\mathrm{H}, 4.520128778,1.5816657239,-0.6517805537$ $\mathrm{H}, 3.9604733951,1.4330820956,1.0331906177$ $\mathrm{H},-2.2476645055,-3.7032551573,2.8750352815$ $\mathrm{H},-2.3135280764,-3.0452698603,1.2170264012$ $\mathrm{H},-0.7941684562,-3.7455664817,1.8400107736$ $\mathrm{H}, 0.2960237259,-0.6121241379,4.6318426433$ $\mathrm{H},-0.5393910265,-2.1184983538,5.099638453$ $\mathrm{H}, 0.8808029543,-2.1840395424,4.0208023714$ $\mathrm{H},-2.3808132275,0.6190587356,3.5202975816$ $\mathrm{H},-3.3253241378,-0.258422247,2.2860596807$ $\mathrm{H},-3.2873504619,-0.8517620581,3.9691694289$ $\mathrm{H},-4.6440572871,-0.6112665276,-3.324968846$ $\mathrm{H},-4.1595547542,0.8247540206,-2.3814302006$ $\mathrm{H},-4.4807474169,-0.7213630792,-1.5511256029$ $\mathrm{H},-1.5537462807,1.1704058451,-4.1320183185$ $\mathrm{H},-0.4111628242,-0.1918385086,-4.3058854501$ $\mathrm{H},-2.0007125442,-0.2534655657,-5.1106478795$ $\mathrm{H},-0.9418761615,-2.8973947964,-2.7894903842$ $\mathrm{H},-2.5259351175,-3.0590544946,-3.5984989733$ $\mathrm{H},-2.423804555,-3.1049452661,-1.8172071196$ $\mathrm{H}, 1.6593987884,0.423010684,-1.7615093253$ C, $-0.3933570427,2.2307734582,0.347232471$ C, $0.5417366087,2.8478518131,1.4212920865$ C, $0.5915119162,4.3508394397,1.0611871507$ C, $0.6693839923,4.3322109749,-0.4789404785$ C, $-0.2653374007,3.1610911044,-0.9071285102$ $\mathrm{H},-1.440173276,2.2625681677,0.7186648844$ $\mathrm{H}, 1.5650262142,2.4292143771,1.3479456412$ $\mathrm{H}, 0.1876038124,2.6561411194,2.4492363021$ $\mathrm{H},-0.3444825391,4.8431506859,1.3883717891$ $\mathrm{H}, 1.4336894349,4.890012192,1.5310060152$ $\mathrm{H}, 0.3855821418,5.293239415,-0.9422282643$ $\mathrm{H}, 1.7089125024,4.1167806217,-0.7885351066$ $\mathrm{H},-1.2604789215,3.5366485372,-1.2081245629$ $\mathrm{H}, 0.1438594946,2.6469969456,-1.7978421088$

\section{$\mathrm{R}=c-\mathrm{Bu}, \mathrm{H}=-791.626223$}

Ti, $0.0687907952,-0.1943377457,0.3557244367$ Si, $-2.7388694243,-1.2035949031,-1.3188371127$ $\mathrm{Si},-0.0771045526,3.2207595359,0.0289104359$ $\mathrm{N},-1.153363201,-1.371998779,-0.5106956432$ $0,-0.3810444647,1.5417601136,0.1456081704$ $C,-1.457548953,4.1165538469,0.9695875061$ C, $-0.0944703092,3.6893220796,-1.8088780176$ C, $1.6151426282,3.5837844331,0.8058645698$ $0,1.7715596881,-0.6551812305,-0.0110836806$ $\mathrm{Si}, 3.2830950668,-1.1245038342,-0.6499194655$ C, 3.2273138577, $-2.9926277206,-0.9771526143$ C, $3.5676299885,-0.1695170581,-2.2641446799$ C, $4.6108626035,-0.7026042839,0.6351707702$ $C,-3.6879953451,0.2116544735,-0.4869109043$ $\mathrm{H},-3.9286718458,-0.0394845874,0.5595711238$
$\mathrm{H},-3.0846348521,1.1342484553,-0.4848376718$ $\mathrm{H},-4.6344075935,0.4171777351,-1.0161343594$ C, $-2.497028851,-0.8231240824,-3.1700141419$ $\mathrm{H},-1.9292624701,0.1136632669,-3.3004884635$ $\mathrm{H},-1.9351450418,-1.6298907306,-3.6713053461$ $\mathrm{H},-3.4646058832,-0.7118852329,-3.6903608965$ C, $-3.6849517643,-2.8463121452,-1.1308357725$ $\mathrm{H},-4.6717603802,-2.7935294829,-1.6229056556$ $\mathrm{H},-3.1318151578,-3.6858950891,-1.5866060209$ $\mathrm{H},-3.8490496277,-3.0858915099,-0.066811704$ $\mathrm{H}, 0.0917168355,4.7687286854,-1.9455830609$ $\mathrm{H}, 0.6824263165,3.1366881999,-2.3632000124$ $\mathrm{H},-1.0707775875,3.4531123497,-2.2639410442$ $\mathrm{H},-1.4757789066,3.8052131711,2.0272103361$ $\mathrm{H},-1.3127817874,5.2104113274,0.9378599831$ $\mathrm{H},-2.4440840886,3.8914180851,0.5314201357$ $\mathrm{H}, 1.6261317086,3.2974210777,1.8708782598$ $\mathrm{H}, 2.4103629223,3.018073348,0.2922958229$ $\mathrm{H}, 1.8628465131,4.6575351069,0.7396209027$ $\mathrm{H}, 5.6143721769,-0.9940665755,0.2795075849$ $\mathrm{H}, 4.4178294094,-1.2310980689,1.583490453$ $\mathrm{H}, 4.6255313558,0.3797053659,0.8458412144$ $\mathrm{H}, 3.0196311402,-3.5479920738,-0.0472970766$ $\mathrm{H}, 2.4405806826,-3.2417214334,-1.7091755434$ $\mathrm{H}, 4.1894576432,-3.3536133437,-1.3802779246$ $\mathrm{H}, 2.7661396571,-0.3820864537,-2.9913734911$ $\mathrm{H}, 4.5301379286,-0.4495732014,-2.7264208737$ $\mathrm{H}, 3.5830496753,0.9177772813,-2.0805355208$ $\mathrm{H},-0.736033265,-2.2992510549,-0.6738325994$ $C_{,}-0.0907299307,-0.5628276588,2.4122332464$ C, $-1.5112395639,-0.4941820466,3.0618231205$ C, $-1.5313078448,-2.0506561887,3.102135022$ C, $0.0083111162,-2.0546800081,2.8716763457$ $\mathrm{H}, 0.6695742753,0.1210906276,2.8461830665$ $\mathrm{H},-2.3219524855,0.0041447819,2.5003660581$ $\mathrm{H},-1.4869694653,-0.0531298288,4.0768104006$ $\mathrm{H},-2.070392691,-2.4705125628,2.2374905252$ $\mathrm{H},-1.9073579012,-2.5298883363,4.0218093127$ $\mathrm{H}, 0.4074263172,-2.8007985256,2.1595255771$ $\mathrm{H}, 0.5706685461,-2.1652630057,3.818461776$

\section{$\mathrm{R}=\mathrm{Cy}, \mathrm{H}=-870.229148$}

Ti, $-0.0819120181,-0.0196308904,-0.121977865$ Si, $-0.5828091526,-2.2168361632,2.4558063325$ Si, $-1.0141560561,-0.7969002031,-3.3914796899$ $\mathrm{N},-0.9772785362,-1.289368551,0.9793839746$ $0,-0.5456703157,-0.2272463981,-1.8514578144$ C, $-2.7832595175,-1.4712903845,-3.2586064601$ C, $0.189304583,-2.1709138713,-3.9038623949$ C, $-0.9345764208,0.6656679144,-4.5945838006$ $0,1.6817877139,0.121077752,0.2443124035$ $\mathrm{Si}, 3.3405249367,0.3426207401,-0.109702082$ C, $4.1460963844,1.1253406516,1.4180154529$ C, $4.095803352,-1.3599072162,-0.4707809738$ C, $3.4922997009,1.4859158999,-1.6159343179$ C, $0.7337596067,-1.2524615983,3.4212202939$ $\mathrm{H}, 1.5766320491,-0.9935516403,2.7593568905$ $\mathrm{H}, 0.3202094586,-0.3130415007,3.8242128291$ $\mathrm{H}, 1.1219927685,-1.8481156835,4.2652463501$ C, $-2.1706846277,-2.416669209,3.4893190449$ $\mathrm{H},-2.5749689952,-1.4331685455,3.7821617833$ $\mathrm{H},-2.9543412221,-2.9535733283,2.9267823686$ $\mathrm{H},-1.9719353342,-2.9911026212,4.4110244459$ C, $0.085349204,-3.9422357087,1.9959796884$ 
$\mathrm{H}, 0.3294422607,-4.5305657381,2.8982290753$ $\mathrm{H},-0.6551035333,-4.5145571545,1.4108407693$ $\mathrm{H}, 1.0017996633,-3.855726394,1.3879448147$ $\mathrm{H},-0.0752626174,-2.5833520874,-4.8931163724$ $\mathrm{H}, 1.2222179943,-1.7885138058,-3.9609859121$ $\mathrm{H}, 0.1706053819,-2.9975456164,-3.1737519977$ $\mathrm{H},-3.4776398516,-0.6894477452,-2.9078253063$ $\mathrm{H},-3.1445818749,-1.8316930563,-4.2376107847$ $\mathrm{H},-2.831745309,-2.3160789191,-2.5506186092$ $\mathrm{H},-1.6003153868,1.4806588514,-4.264742283$ $\mathrm{H}, 0.090418784,1.0680186003,-4.6555621393$ $\mathrm{H},-1.2429498597,0.3630901,-5.6104393882$ $\mathrm{H}, 4.5501284417,1.6434058005,-1.8898795489$ $\mathrm{H}, 3.0448048312,2.4721641841,-1.4071503962$ $\mathrm{H}, 2.9746811798,1.0550020841,-2.4892956244$ $\mathrm{H}, 3.6973692212,2.1078105482,1.6406771976$ $\mathrm{H}, 4.014210744,0.4831791032,2.3048792126$ $\mathrm{H}, 5.2291579707,1.2725634562,1.2627390676$ $\mathrm{H}, 3.9812595586,-2.0299540993,0.3976910908$ $\mathrm{H}, 5.1734077399,-1.2765402265,-0.6958209288$ $\mathrm{H}, 3.6032969445,-1.835740455,-1.3353429144$ $\mathrm{H},-1.8647983036,-1.5962255696,0.5556577579$ $C,-3.0069314165,3.331474069,0.4445852532$ $C_{,}-2.4343648394,1.9275072395,0.1345445505$ C, $-0.9157809875,1.8464060272,0.4009551001$ $C_{,}-0.5869274343,2.2976567766,1.8393702249$ $C_{,}-1.1572953652,3.7017850428,2.1538228596$ $C_{,}-2.6727762539,3.7648213071,1.8846227786$ $\mathrm{H},-2.9547727857,1.1877200249,0.7780231778$ $\mathrm{H},-2.6639463547,1.6517667145,-0.9116900898$ $\mathrm{H},-2.5721478251,4.0622649809,-0.266748864$ $\mathrm{H},-4.1019833145,3.3449222862,0.282729517$ $\mathrm{H},-1.0239615526,1.5706478107,2.5547452271$ $\mathrm{H}, 0.5049953006,2.2892463074,2.0133344382$ $\mathrm{H},-0.9410252463,3.9785149806,3.2035808567$ $\mathrm{H},-0.6436688752,4.4498176034,1.5167163777$ $\mathrm{H},-3.1937597572,3.0900251114,2.5942287824$ $\mathrm{H},-3.0584525082,4.7826100295,2.0814095299$ $\mathrm{H},-0.3951268219,2.5289113485,-0.3133895526$

\section{$\mathrm{R}=$ Benzyl, $\mathrm{H}=-\mathbf{9 0 5 . 9 6 4 6 8 5}$}

Ti, $-0.4630930583,0.2158509005,-0.4022434606$ $\mathrm{Si},-3.7314319783,-0.6475233062,-0.801507191$ Si, $0.5522033594,-1.8209378605,2.1474246127$ $\mathrm{N},-2.2843416156,0.401896897,-0.9036673629$ $0,-0.2495184294,-1.0994389698,0.8098154206$ C, $0.6994105478,-3.6737574675,1.7796705431$ C, $-0.5245661716,-1.512977342,3.679694898$ C, $2.260559015,-1.0266837386,2.3305475822$ $0,0.2173334365,1.8394068782,-0.0364155472$ Si, $0.5506993117,3.4184640636,0.5325493191$ C, $0.4363007906,4.5932843813,-0.952132809$ C, $-0.7468079247,3.851975673,1.8461305885$ C, $2.2971811965,3.4233670871,1.267033451$ C, $-3.1419415427,-2.4489550904,-0.754080047$ $\mathrm{H},-2.6975237918,-2.744153668,-1.7195679053$ $\mathrm{H},-2.3752059809,-2.5774492064,0.0279138866$ $\mathrm{H},-3.9781928522,-3.135881911,-0.5379112612$ C, $-4.7190974895,-0.2354648739,0.7731131585$ $\mathrm{H},-4.0950892117,-0.3937923381,1.6688903639$ $\mathrm{H},-5.0458427996,0.8186917664,0.7721826147$ $\mathrm{H},-5.6194423377,-0.8681396488,0.8656726941$ $C,-4.8109739691,-0.3234674012,-2.3366107275$ $\mathrm{H},-5.7225837869,-0.9459830833,-2.3169672503$
$\mathrm{H},-5.1319259234,0.7315859338,-2.3890561853$ $\mathrm{H},-4.2586831593,-0.5572475959,-3.2622981248$ $\mathrm{H},-0.0787997324,-1.9762827913,4.5772182795$ $\mathrm{H},-0.63358916,-0.4325560818,3.8739004599$ $\mathrm{H},-1.5331818137,-1.93795306,3.543524126$ $\mathrm{H}, 1.306091389,-3.8344728376,0.8733423231$ $\mathrm{H}, 1.1869505037,-4.2040342106,2.6162827816$ $\mathrm{H},-0.2915162928,-4.1299017812,1.617856461$ $\mathrm{H}, 2.8439637368,-1.1523659803,1.4029346367$ $\mathrm{H}, 2.1731438255,0.0530002359,2.5378514794$ $\mathrm{H}, 2.8249176107,-1.4882003059,3.1598505312$ $\mathrm{H}, 2.584624886,4.4356615737,1.6010063142$ $\mathrm{H}, 3.0351537974,3.0854909292,0.5209196292$ $\mathrm{H}, 2.3620078208,2.748044551,2.1365384434$ $\mathrm{H}, 1.1625052091,4.3100961191,-1.7323624324$ $\mathrm{H},-0.5713722449,4.5705629768,-1.400402697$ $\mathrm{H}, 0.6497284033,5.6335614404,-0.6506081121$ $\mathrm{H},-1.7631982975,3.8283643374,1.4183518043$ $\mathrm{H},-0.5736844811,4.8615462401,2.2580437288$ $\mathrm{H},-0.7130136631,3.133446322,2.6822469277$ C, $0.5001520424,-0.428673387,-2.1936112114$ $\mathrm{H},-0.099150979,-1.2702997222,-2.5840447164$ $\mathrm{H}, 0.4016220719,0.4290917902,-2.8850231496$ $\mathrm{H},-2.5063125452,1.3462237762,-1.248293753$ C, $1.9190545512,-0.8041886862,-1.9363176171$ C, $2.9475511578,0.17129351,-1.8856241163$ C, $4.2764559979,-0.1808157041,-1.6086094683$ C, $4.6242715492,-1.5217238087,-1.3714441963$ C, $3.6213452936,-2.5058560612,-1.4232958059$ C, $2.2931381957,-2.152323336,-1.7006509733$ $\mathrm{H}, 2.6906933888,1.2204596628,-2.0735563763$ $\mathrm{H}, 5.0483092977,0.5968914684,-1.5843207426$ $\mathrm{H}, 5.6624059559,-1.7972197059,-1.1590236474$ $\mathrm{H}, 3.8774986412,-3.5583611755,-1.2563927211$ $\mathrm{H}, 1.521821909,-2.9304084619,-1.7482835898$

\section{$\mathrm{R}=\mathrm{CH}_{2} \mathrm{SiMe}_{3}, \mathrm{H}=-797.972916$}

Ti, $-0.1146993515,0.2152754962,-0.400637679$ $\mathrm{Si},-3.348732812,0.6921495506,-1.3343336446$ $\mathrm{Si},-0.7333768489,-1.0438286327,2.7296023998$ $\mathrm{N},-1.6192518556,1.0992631391,-1.1597858728$ $0,-0.5851299579,-0.7560504201,1.0484615372$ C, $-1.9857307448,-2.4493124851,2.9546696848$ C, $-1.3467931492,0.5499632545,3.5572719711$ C, $0.9666159037,-1.5400307164,3.4080587664$ $0,1.1875616766,1.4278193633,-0.1716446397$ $\mathrm{Si}, 2.2747916448,2.7087872784,0.1519556135$ C, $3.1954422835,3.0985308401,-1.4586011761$ C, $1.258016446,4.2008852679,0.7339254817$ C, $3.4761337701,2.1388733944,1.5025593826$ C, $-3.5342279476,-1.1808579545,-1.0980104572$ $\mathrm{H},-3.0257135061,-1.7301099833,-1.9082614478$ $\mathrm{H},-3.0900936098,-1.5004697657,-0.1405806748$ $\mathrm{H},-4.597705501,-1.4761330634,-1.1001441974$ $C,-4.3868474212,1.6229524415,-0.0356281552$ $\mathrm{H},-4.0755462445,1.3439403043,0.9852287519$ $\mathrm{H},-4.2631927673,2.7149556773,-0.1373464816$ $\mathrm{H},-5.4621788812,1.3938792556,-0.1394302335$ C, $-3.9197418832,1.209857862,-3.0766191226$ $\mathrm{H},-4.990081291,0.9841863584,-3.2267078903$ $\mathrm{H},-3.7837273136,2.2935072288,-3.2388572415$ $\mathrm{H},-3.3461165997,0.6758972513,-3.8527313088$ $\mathrm{H},-1.5155521309,0.3965963105,4.6375502465$ $\mathrm{H},-0.6103776167,1.3628500262,3.4408267087$ 
$\mathrm{H},-2.2966416984,0.8846273763,3.1077704323$ $\mathrm{H},-1.654224634,-3.3583967988,2.4257478038$ $\mathrm{H},-2.1081784279,-2.7021260269,4.0223470619$ $\mathrm{H},-2.9748963815,-2.1656965723,2.5578852488$ $\mathrm{H}, 1.3298733574,-2.4631815972,2.9268703038$ $\mathrm{H}, 1.7093731189,-0.7450790641,3.2260910929$ $\mathrm{H}, 0.9200305089,-1.7188252791,4.4966013871$ $\mathrm{H}, 4.2168146062,2.9250213471,1.7309607663$ $\mathrm{H}, 4.0223564833,1.2360355816,1.1829723892$ $\mathrm{H}, 2.9363189392,1.9014069281,2.4346891532$ $\mathrm{H}, 3.7524766252,2.2156049594,-1.8136938048$ $\mathrm{H}, 2.4923081855,3.4001666063,-2.2530549639$ $\mathrm{H}, 3.9174718309,3.9207535344,-1.3125485152$ $\mathrm{H}, 0.5296623523,4.5084192689,-0.035237321$ $\mathrm{H}, 1.9112096169,5.0651566011,0.9466692241$ $\mathrm{H}, 0.7002434889,3.961940941,1.654860482$ C, $0.5622355965,-1.118667709,-1.8729671997$
$\mathrm{H},-0.337258758,-1.6250820318,-2.2786233613$ $\mathrm{H}, 0.9565891647,-0.4701692734,-2.6830292052$ $\mathrm{H},-1.3820316339,2.0159660844,-1.5616476621$ $\mathrm{Si}, 1.8502563672,-2.4345860435,-1.4134585286$ C, $3.2636843274,-1.6738852805,-0.3770877509$ $\mathrm{H}, 4.0197215601,-2.4318260456,-0.1070622819$ $\mathrm{H}, 3.7743696344,-0.8730273137,-0.9395406597$ $\mathrm{H}, 2.8736107118,-1.2330252098,0.5560610935$ C, $1.0072390353,-3.8241222255,-0.4131031241$ $\mathrm{H}, 1.7342513977,-4.5792527416,-0.0673700179$ $\mathrm{H}, 0.4955456724,-3.4052963653,0.4694745174$ $\mathrm{H}, 0.2492309707,-4.3423904642,-1.0259166553$ C, $2.6217792625,-3.2032070208,-2.9882706869$ $\mathrm{H}, 3.1345375826,-2.4370722077,-3.5954695222$ $\mathrm{H}, 3.3620075374,-3.98292221,-2.7353147031$ $\mathrm{H}, 1.8462388331,-3.6670722132,-3.6219510919$ 


\section{Cartesian coordinates for the chloride complexes $\mathbf{R h}(\mathrm{Cl})(\mathrm{R})(\mathrm{Tp})\left(\mathrm{CNCH}_{3}\right)$ and en- thalpie at B3PW91}

\author{
$\mathrm{R}=\mathrm{Et}, \mathrm{H}=-1039.450151$
}

$\mathrm{Rh}, 0.3917865092,-0.2051034244,0.5441872912$ $\mathrm{N}, 1.2090586576,-0.4476799312,-1.3678221988$ $\mathrm{N}, 0.5352617043,0.0480648999,-2.428737944$ $\mathrm{N},-1.4809909391,-0.8808840974,-0.433907291$ $\mathrm{N},-1.8028203193,-0.2983233325,-1.6068360681$ $\mathrm{N},-0.1147600221,1.6994364496,-0.0242324762$ $\mathrm{N},-0.6475708982,1.9045979533,-1.2454396848$ $\mathrm{N},-0.9340391556,0.0046670991,3.2943034581$ C, $2.2899627274,-1.074855224,-1.8316704906$ C, $2.3311637274,-0.9789005651,-3.2285784258$ $\mathrm{H}, 3.0788698947,-1.3795773828,-3.8959848464$ C, $1.194916306,-0.2609083888,-3.5635037111$ C, $-2.4125532203,-1.7974630892,-0.1778888906$ $C,-3.370504701,-1.8117853313,-1.2034337565$ $\mathrm{H},-4.2442286539,-2.4405392158,-1.2870466689$ C, $-2.9380172638,-0.8437199901,-2.0941915209$ $C_{,}-0.0460043353,2.8826921617,0.5927720578$ C, $-0.5480524175,3.8837461761,-0.2462174022$ $\mathrm{H},-0.6270194752,4.9404383213,-0.0408516715$ C, $-0.9160105702,3.2153687905,-1.4033119044$ C, $2.2433243798,0.447723371,1.210763854$ $\mathrm{H}, 2.4283342184,1.3937659702,0.687411903$ C, $-0.4035304091,-0.0480952043,2.2537338$ $C,-1.5194676201,-0.0259044525,4.5780826783$ $\mathrm{H},-2.5157039964,0.4234466195,4.5458662827$ $\mathrm{H},-0.8977934613,0.5315052143,5.2844569273$ $\mathrm{B},-0.8374068908,0.7260513742,-2.2317110739$ $\mathrm{H},-1.2644524259,1.1312760718,-3.2796103753$ $\mathrm{H},-3.3488125536,-0.5088942157,-3.0358332929$ $\mathrm{H},-2.3324975307,-2.4085224344,0.7101851409$ $\mathrm{H}, 0.8060420649,0.0489603921,-4.5225694454$ $\mathrm{H}, 2.9587000071,-1.5737490973,-1.1458150187$ $\mathrm{H},-1.3452637651,3.5756779708,-2.3266198186$ $\mathrm{H}, 0.3623454226,2.9503958247,1.5905360814$ $\mathrm{H},-1.6036375221,-1.0617137976,4.918256122$ $\mathrm{H}, 2.9388061259,-0.2990495607,0.816353339$ C, $2.4751625787,0.6016070993,2.7056386178$ $\mathrm{H}, 1.8358543834,1.3719339664,3.154798322$ $\mathrm{H}, 2.2929089801,-0.3413192611,3.2314823714$ $\mathrm{H}, 3.5153624338,0.8936079326,2.9086787793$ $\mathrm{Cl}, 0.993383477,-2.4114371467,1.1465864195$

$$
\mathrm{R}=i \text { - } \mathrm{Pr}, \mathrm{H}=-1078.714625
$$

$\mathrm{Rh},-0.6002923272,-0.1921120091,-0.1658799797$ $\mathrm{N}, 1.1480477192,-1.2943146996,-0.5299826864$ $\mathrm{N}, 2.3292203478,-0.8145146781,-0.0825632421$ $\mathrm{N}, 0.6483006482,1.4783406321,-0.9456061639$ $\mathrm{N}, 1.8824523069,1.6180258163,-0.4204315271$ $\mathrm{N}, 0.1464238956,0.2833251634,1.6949464787$ $\mathrm{N}, 1.4582036543,0.5714564822,1.8185990295$ $\mathrm{N},-3.1316232984,1.5003052481,0.132639938$ C, $1.4034425106,-2.3853601548,-1.251635305$ C, $2.7814742624,-2.6359412112,-1.2646303592$ $\mathrm{H}, 3.3005894573,-3.4467029162,-1.7527536803$ C, $3.3300990235,-1.6089668161,-0.5146342767$ C, $0.4921229669,2.4422198132,-1.8512527635$ C, $1.6487191677,3.2346841597,-1.9174792505$ $\mathrm{H}, 1.8334147357,4.0879498441,-2.5527820383$ C, $2.5090588368,2.6691646254,-0.9912710453$
C, $-0.411283618,0.4211877105,2.9019697749$ C, $0.5595554296,0.8023723917,3.8341607472$ $\mathrm{H}, 0.4259568668,0.986912504,4.8891886866$ C, $1.7315008534,0.8858285769,3.0997701191$ C, $-1.6606198063,-1.780480802,0.7432761111$ $\mathrm{H},-2.1586047976,-1.2833585719,1.5883669371$ $C_{,}-2.164306062,0.8491709923,0.0401087831$ C, $-4.3132728945,2.2729924233,0.1118386037$ $\mathrm{H},-4.0823762967,3.3135891412,0.3555980155$ $\mathrm{H},-5.0268691305,1.8848582173,0.8436883667$ $\mathrm{B}, 2.3964696471,0.5720468583,0.5879867217$ $\mathrm{H}, 3.5204183767,0.824438217,0.9312795346$ $\mathrm{H}, 3.51691304,2.9311871575,-0.7028495354$ $\mathrm{H},-0.425504875,2.4992387215,-2.4196656967$ $\mathrm{H}, 4.3555149695,-1.3807714152,-0.2626387036$ $\mathrm{H}, 0.5918726411,-2.9130576307,-1.7305802638$ $\mathrm{H}, 2.7380150624,1.1454649922,3.3930914982$ $\mathrm{H},-1.465548071,0.2316039464,3.0403193977$ $\mathrm{H},-4.7621694647,2.2277375024,-0.8841857822$ C, $-2.7442054358,-2.422957212,-0.1134706093$ $\mathrm{H},-3.4621849549,-1.694788828,-0.5006049093$ $\mathrm{H},-2.3241386788,-2.9435291834,-0.9784207504$ $\mathrm{H},-3.2990143623,-3.1608740917,0.4867569769$ C, $-0.734930782,-2.8418837975,1.3301967268$ $\mathrm{H}, 0.0546322477,-2.4147078548,1.9549168576$ $\mathrm{H},-1.3183354666,-3.5329344222,1.9571986577$ $\mathrm{H},-0.2557378486,-3.4438384424,0.5532593069$ $\mathrm{Cl},-1.3327847406,-0.6437742773,-2.3705537484$

$$
\mathrm{R}=t \text {-Bu-vinyl, } \mathrm{H}=-1195.330324
$$

$\mathrm{Rh}, 0.3173015543,-0.2675479185,0.0744578086$ $\mathrm{N},-1.6558330801,-0.5490117396,0.6859183227$ $\mathrm{N},-2.0580868018,-0.0353283214,1.8680287466$ $\mathrm{N}, 0.8172981469,-0.8137097182,2.1424128502$ $\mathrm{N}, 0.0994734432,-0.2315651864,3.1250994252$ $\mathrm{N}, 0.1264608474,1.6677027134,0.743225636$ $\mathrm{N},-0.4763592479,1.8967714027,1.9272125172$ $\mathrm{N}, 3.2069794187,0.0579444675,-0.8921306616$ $\mathrm{C},-2.6867673947,-1.2006043995,0.1496078502$ C, $-3.7956942332,-1.1077300847,1.0012717957$ $\mathrm{H},-4.7790554412,-1.5272599213,0.8525921311$ C, $-3.3494946638,-0.3611168209,2.0799351187$ C, $1.6741252644,-1.6536711027,2.720818371$ C, $1.5189315393,-1.6158493963,4.1146161177$ H, $2.0625121482,-2.1792341795,4.8580488797$ C, $0.5020757242,-0.699677617,4.3255493291$ C, $0.4951635904,2.8481797991,0.2375875507$ C, $0.125368458,3.8731711274,1.1155375091$ $\mathrm{H}, 0.280083973,4.9345467652,0.9948402702$ C, $-0.4898660271,3.2212887968,2.1729166543$ $C,-0.3330557167,0.3263175821,-1.7435313541$ $\mathrm{H},-1.0053599334,1.1838831719,-1.6644843965$ C, $-0.0657642992,-0.2020312184,-2.9408165989$ $\mathrm{H}, 0.5884077407,-1.0740292215,-2.9972727888$ C, $2.1057901654,-0.0486653375,-0.5175371435$ C, $4.526879702,0.0886907672,-1.3918327822$ $\mathrm{H}, 5.1904021167,0.5627780067,-0.6636645835$ $\mathrm{H}, 4.5569109585,0.6546738382,-2.3267439078$ $\mathrm{B},-1.0524925821,0.7228491811,2.7601391324$ $\mathrm{H},-1.5906780231,1.143431295,3.7487886193$ $\mathrm{H}, 0.0343164014,-0.352342211,5.2354743765$ 
$\mathrm{H}, 2.3327591193,-2.2531864715,2.1085316555$ $\mathrm{H},-3.8530276279,-0.0407276839,2.9804384245$ $\mathrm{H},-2.5647231638,-1.7010011837,-0.7997696259$ $\mathrm{H},-0.9380474008,3.6021073541,3.0788577727$ $\mathrm{H}, 0.9887607431,2.8933440437,-0.722329125$ $\mathrm{H}, 4.8702684123,-0.9321253205,-1.5801717613$ C, $-0.6136282022,0.2434787285,-4.2883379619$ $C,-1.4035862773,-0.9315096458,-4.8966256253$ $\mathrm{H},-0.7799106393,-1.82913722,-4.9716131016$ $\mathrm{H},-2.2712497114,-1.1808140469,-4.2771186153$ $\mathrm{H},-1.761424985,-0.683180556,-5.9034344064$ C, $-1.5269738938,1.468828679,-4.1895682597$ $\mathrm{H},-1.8977180459,1.7484308492,-5.1821734836$ $\mathrm{H},-2.3954370453,1.2709744706,-3.5524112742$ $\mathrm{H},-0.9951182439,2.332906605,-3.7761937772$ C, $0.576431707,0.5711533465,-5.2096811756$ $\mathrm{H}, 1.1536864393,1.4158944525,-4.8176087541$ $\mathrm{H}, 1.2525486145,-0.2867535784,-5.2994708098$ $\mathrm{H}, 0.231588601,0.83335945,-6.2172853169$ $\mathrm{Cl}, 0.4878974246,-2.5283689899,-0.5820557458$

\section{$\mathrm{R}=$ Me-vinyl, $\mathrm{H}=-1077.513197$}

$\mathrm{Rh},-0.0172234589,-0.2216972279,0.5154311174$ $\mathrm{N}, 1.4957374012,-0.5489191741,-0.8790445163$ $\mathrm{N}, 1.3619079899,-0.049818346,-2.1263446634$ $\mathrm{N},-1.365106616,-0.7683407201,-1.1303956969$ $\mathrm{N},-1.1292617816,-0.2067710653,-2.3340831204$ $\mathrm{N},-0.0977698905,1.705553207,-0.197142068$ $\mathrm{N},-0.0591699588,1.9151791888,-1.5285588934$ $\mathrm{N},-2.1941593994,0.1830897845,2.6341997858$ C, $2.6473663744,-1.2164690075,-0.8249748565$ C, $3.2872888635,-1.1500922108,-2.0698558094$ $\mathrm{H}, 4.2336981787,-1.5873230918,-2.3495468529$ C, $2.4341487964,-0.4017104118,-2.8649575544$ $C_{,}-2.4008484079,-1.5934794411,-1.2730124036$ C, $-2.8589069347,-1.5665087117,-2.5987956378$ $\mathrm{H},-3.6783232008,-2.1225684797,-3.0288397579$ $C,-2.0161617725,-0.6729858637,-3.2386950134$ $\mathrm{C},-0.1944361614,2.8962809608,0.401215854$ C, $-0.2225672845,3.9081700968,-0.564894523$ $\mathrm{H},-0.2936284478,4.9731788029,-0.4044533906$ C, $-0.1324554993,3.2375969081,-1.7747513432$ C, $1.361371543,0.3599564926,1.8684020189$ $\mathrm{H}, 1.9573892743,1.2070354928,1.511174165$ C, $1.6376368506,-0.1549533013,3.0703205954$ $\mathrm{H}, 1.0707532731,-1.0142239072,3.4286116639$ $C_{,}-1.3684022909,0.0439276342,1.8199122453$ $C,-3.1607520544,0.2591617144,3.6601340062$ $\mathrm{H},-4.0581185268,0.7625811452,3.2906173086$ $\mathrm{H},-2.7584083134,0.8195810456,4.5083577489$ $B, 0.0843968718,0.7246412295,-2.5118413417$ $\mathrm{H}, 0.1540398392,1.1288233813,-3.6412932837$ $\mathrm{H},-1.9789943919,-0.3406452979,-4.2661206109$ $\mathrm{H},-2.7418935697,-2.1752327505,-0.4284510859$ $\mathrm{H}, 2.5091739593,-0.0970857551,-3.8987371016$ $\mathrm{H}, 2.9350696851,-1.7075486616,0.0930618278$ $\mathrm{H},-0.1114864438,3.6039712367,-2.7906187901$ $\mathrm{H},-0.2262052895,2.9576277764,1.4793808705$ $\mathrm{H},-3.4243120561,-0.7493317344,3.9901146514$ C, $2.7312776956,0.343560036,3.9741392405$ $\mathrm{H}, 3.2636894128,1.1925550558,3.5324758321$ $\mathrm{H}, 2.335244866,0.6619835878,4.947694819$ $\mathrm{H}, 3.4660558785,-0.4456296988,4.1803704311$ $\mathrm{Cl}, 0.067454391,-2.4752422667,1.2138512515$

\section{$\mathrm{R}=$ vinyl, $\mathrm{H}=-1038.236170$}

$\mathrm{Rh},-0.3639111604,-0.1937701813,-0.5765659257$ $\mathrm{N},-1.1783296233,-0.5287987494,1.3118319401$ $\mathrm{N},-0.538576716,-0.0491843781,2.3998372266$ $\mathrm{N}, 1.5331453663,-0.774199822,0.3672100027$ $\mathrm{N}, 1.8170153644,-0.2282822133,1.5678061929$ $\mathrm{N}, 0.0268427937,1.7221807884,0.0580083156$ $\mathrm{N}, 0.5386369246,1.9129987014,1.2907516205$ $\mathrm{N}, 0.7690655361,0.211610219,-3.3959045726$ C, $-2.2573608214,-1.1904842946,1.727871127$ C, $-2.3284997608,-1.1398387583,3.1263539596$ $\mathrm{H},-3.0812475206,-1.5765244329,3.7648996299$ C, $-1.2161995887,-0.4076440629,3.5094448173$ C, $2.5261331607,-1.6096993116,0.0664854212$ C, $3.4854126949,-1.6060361313,1.0900671094$ $\mathrm{H}, 4.4014085146,-2.1750288566,1.1425527162$ C, $2.989218114,-0.7152311462,2.0271080841$ C, $-0.1081308792,2.9202094111,-0.5182887616$ C, $0.3287422961,3.9175498278,0.3605647635$ $\mathrm{H}, 0.3463088565,4.9837890869,0.1937081106$ C, $0.7284654608,3.2307424188,1.4964290754$ C, $-2.1636054145,0.418583806,-1.2413483405$ $\mathrm{H},-2.5452502861,1.2655024225,-0.6629924064$ C, $-2.9093176913,-0.0730192416,-2.2325914757$ $\mathrm{H},-2.5986364552,-0.9299457203,-2.8243083693$ C, $0.3443316839,0.0743790955,-2.3167800207$ C, $1.2325249588,0.2781689058,-4.7276942355$ $\mathrm{H}, 2.2276333454,0.7300545356,-4.7543724836$ $\mathrm{H}, 0.5472144348,0.8815233883,-5.3290527686$ B, $0.7947543452,0.7101155165,2.2356288814$ $\mathrm{H}, 1.1990016155,1.099703442,3.2980464216$ $\mathrm{H}, 3.3789023433,-0.3976029735,2.9834981266$ $\mathrm{H}, 2.4864521152,-2.1823219273,-0.8492678163$ $\mathrm{H},-0.856909528,-0.1181897311,4.486303289$ $\mathrm{H},-2.9020460986,-1.6670669111,1.0038930372$ $\mathrm{H}, 1.1299676606,3.5826557143,2.4353489752$ $\mathrm{H},-0.5162474917,2.9970295057,-1.5157846308$ $\mathrm{H}, 1.2839874755,-0.7302972835,-5.1469161453$ $\mathrm{H},-3.8870074273,0.3537775,-2.4602404806$ $\mathrm{Cl},-0.7585990507,-2.4351960067,-1.200029834$

\section{$\mathrm{R}=c-\mathrm{Pr}, \mathrm{H}=-1077.499725$}

$\mathrm{Rh}, 0.1694778732,-0.1776240964,0.5500044046$ $\mathrm{N}, 1.341841632,-0.4433604111,-1.1617538758$ $\mathrm{N}, 0.8755208263,0.0131944533,-2.3451969827$ $\mathrm{N},-1.4766793874,-0.8691192601,-0.7370204839$ $\mathrm{N},-1.5757226117,-0.3201181871,-1.9645855008$ $\mathrm{N},-0.2181404159,1.7148294748,-0.1453714231$ $\mathrm{N},-0.5067974046,1.8912760888,-1.4501201808$ $\mathrm{N},-1.5530666346,0.1480408141,3.0609261492$ C, $2.510519925,-1.040764684,-1.3902521035$ C, $2.820628437,-0.969657949,-2.7546390022$ $\mathrm{H}, 3.6958311846,-1.3572192157,-3.2535309139$ C, $1.753188801,-0.293615683,-3.3219754356$ C, $-2.4520037114,-1.7692328271,-0.624096253$ C, $-3.2115937358,-1.8060798608,-1.8032706577$ $\mathrm{H},-4.0652235207,-2.4293913111,-2.0233133428$ $C,-2.6139548534,-0.8694972809,-2.6300744079$ C, $-0.2844833875,2.9087362798,0.4515874893$ $C,-0.6245539726,3.8880539368,-0.4882055278$ $\mathrm{H},-0.7529278744,4.9468470988,-0.3224406486$ C, $-0.7542701409,3.1951116745,-1.6816627455$ $C_{,},-0.8959942978,0.0390393657,2.1007019879$ C, $-2.3093597735,0.1768558991,4.2525728646$ 
$\mathrm{H},-3.3043600119,0.5838475151,4.0534176183$ $\mathrm{H},-1.8086305496,0.8027314389,4.9962787496$ $\mathrm{B},-0.5079364051,0.6883444187,-2.4276324821$ $\mathrm{H},-0.7328529259,1.0668657962,-3.5458590283$ $\mathrm{H},-2.8459863757,-0.559477351,-3.6387952151$ $\mathrm{H},-2.5391411513,-2.3533918706,0.2810658784$ $\mathrm{H}, 1.5522621956,-0.0110952785,-4.3451512998$ $\mathrm{H}, 3.0517586042,-1.4888988417,-0.5713591345$ $\mathrm{H},-1.0006128812,3.5338530939,-2.6772394623$ $\mathrm{H},-0.0781849599,2.9974298133,1.5084093457$ $\mathrm{H},-2.4084823706,-0.83785497,4.6476907641$ C, $1.7870967072,0.5511367487,1.5822218094$ C, $2.131567054,0.2705142254,3.0205041766$ C, $3.0286426932,-0.2292991681,1.9155911087$ $\mathrm{H}, 1.9845095206,1.575081831,1.2626079879$ $\mathrm{H}, 1.5641238943,-0.4930341526,3.5460449611$ $\mathrm{H}, 2.4756327266,1.0892869273,3.6500350051$ $\mathrm{H}, 3.9921189361,0.2540991897,1.7660207886$ $\mathrm{H}, 3.0267549622,-1.3034378783,1.7569260704$ $\mathrm{Cl}, 0.5638422256,-2.3950520272,1.2779407262$

\section{$\mathrm{R}=\mathrm{Pe}, \mathrm{H}=-1157.266384$}

$\mathrm{Rh}, 0.1342594086,-0.3404602433,0.0023186049$ $\mathrm{N},-1.7837658793,-0.4867164931,0.8229596352$ $\mathrm{N},-2.0255132581,0.0945948154,2.0182429494$ $\mathrm{N}, 0.7936563515,-0.883845935,2.046304196$ $\mathrm{N}, 0.2234292755,-0.2174237054,3.0708217342$ $\mathrm{N}, 0.1402786091,1.6150489902,0.6280970671$ $\mathrm{N},-0.3213502422,1.9152205181,1.8586056931$ $\mathrm{N}, 3.0038032595,-0.2594732012,-1.0598517429$ C, $-2.8945031819,-1.1221697382,0.4494397944$ $C,-3.8906842576,-0.9449258379,1.4181266156$ $\mathrm{H},-4.8986329332,-1.3308524782,1.406499919$ C, $-3.2923769499,-0.1692399848,2.3974837137$ C, $1.6351162328,-1.772768081,2.5711629029$ C, $1.6202944418,-1.6830628752,3.9715730181$
$\mathrm{H}, 2.1880483762,-2.2671460678,4.6802362411$ C, $0.7030822481,-0.6821443954,4.2444579689$ C, $0.5379613128,2.7544939554,0.0544578393$ C, $0.331734056,3.8237948326,0.933284993$ $\mathrm{H}, 0.5474046554,4.8680634979,0.7659367192$ C, $-0.2176715431,3.2425616328,2.0652247919$ $C,-0.720055484,0.2072974414,-1.805429923$ $\mathrm{H},-1.1928749651,1.1819858378,-1.6268234345$ C, $1.8970531718,-0.2652762664,-0.6825583203$ C, $4.3166446597,-0.3510795746,-1.5695284214$ $\mathrm{H}, 5.0223095499,0.102257867,-0.8681169314$ $\mathrm{H}, 4.3812607188,0.1697870101,-2.5290287356$ $\mathrm{B},-0.8887649352,0.8079633052,2.780580713$ $\mathrm{H},-1.3002262417,1.2926160996,3.8005910288$ $\mathrm{H}, 0.3540887133,-0.2730656419,5.1816901868$ $\mathrm{H}, 2.1839211962,-2.4397829025,1.9212297357$ $\mathrm{H},-3.671993815,0.2107064935,3.3348060026$ $\mathrm{H},-2.9093566063,-1.6830949114,-0.4734615074$ $\mathrm{H},-0.5447241016,3.6750862684,2.9993102745$ $\mathrm{H}, 0.9373744884,2.7450333186,-0.9493133615$ $\mathrm{H}, 4.5805411161,-1.402105591,-1.7156475099$ $\mathrm{H},-1.5164183089,-0.5300295333,-1.9534239727$ C, $0.1468455778,0.255144795,-3.0560678699$ $\mathrm{H}, 0.9432360183,1.007478637,-2.9471868186$ $\mathrm{H}, 0.6453215519,-0.7134851168,-3.1911030371$ $C,-0.6578546232,0.5811280964,-4.32035771$ $\mathrm{H},-1.1809076621,1.5392088834,-4.1846915086$ $\mathrm{H},-1.4429365747,-0.1770805342,-4.4496836378$ C, $0.1947152758,0.6460837429,-5.5887046831$ $\mathrm{H}, 0.9787344657,1.4057740626,-5.4592609787$ $\mathrm{H}, 0.7187537661,-0.3107435542,-5.7190998469$ C, $-0.6144984438,0.9591667965,-6.8453379237$ $\mathrm{H},-1.1260659781,1.9245557743,-6.7573320872$ $\mathrm{H}, 0.0218994596,1.0007646926,-7.7356877906$ $\mathrm{H},-1.3814923772,0.1964605428,-7.0209634067$ $\mathrm{Cl}, 0.0750767651,-2.6016094347,-0.6844902218$ 